\title{
Diversification of multipotential postmitotic mouse retinal ganglion cell precursors into discrete types
}

Karthik Shekhar ${ }^{1,2,3,4^{*}}$, Irene E. Whitney ${ }^{4,5}$, Salwan Butrus ${ }^{1}$, Yi-Rong Peng ${ }^{4,6}$, and Joshua R. Sanes ${ }^{4^{*}}$

Author Affiliations:

1. Department of Chemical and Biomolecular Engineering; Helen Wills Neuroscience Institute; Center for Computational Biology; California Institute for Quantitative Biosciences, QB3, University of California, Berkeley, CA, USA 94720

2. Biological Systems and Engineering Division, Lawrence Berkeley National Laboratory, Berkeley, CA USA 94720

3. Broad Institute of Harvard and MIT, Cambridge, MA 02142, USA

4. Center for Brain Science and Department of Molecular and Cellular Biology, Harvard University, Cambridge MA, 02138

5. Present address: Honeycomb Biotechnologies 940 Winter Street Waltham MA 02451

6. Department of Ophthalmology, Stein Eye Institute, UCLA David Geffen School of Medicine, Los Angeles, CA 90095, USA

*Correspondence

Karthik Shekhar (kshekhar@berkeley.edu) Joshua R Sanes (sanesj@mcb.harvard.edu) 
Abstract (149 words)

39 The genesis of broad neuronal classes from multipotential neural progenitor cells has

40 been extensively studied, but less is known about the diversification of a single neuronal

41 class into multiple types. We used single-cell RNA-seq to study how newly-born

42 (postmitotic) mouse retinal ganglion cell (RGC) precursors diversify into $\sim 45$ discrete

43 types. Computational analysis provides evidence that RGC transcriptomic type identity

44 is not specified at mitotic exit, but acquired by gradual, asynchronous restriction of

45 postmitotic multipotential precursors. Some types are not identifiable until a week after

46 they are generated. Immature RGCs may be specified to project ipsilaterally or

47 contralaterally to the rest of the brain before their type identity emerges. Optimal

48 transport inference identifies groups of RGC precursors with largely non-overlapping

49 fates, distinguished by selectively expressed transcription factors that could act as fate

50 determinants. Our study provides a framework for investigating the molecular

51 diversification of discrete types within a neuronal class.

52

53 Keywords: development, diversification, retina, retinal ganglion cell, single-cell RNA-

54 seq, optimal transport

55

56

57

58

59

60

61

62

63

64

65

66

67

68

69

70

71

72 


\section{Introduction}

A central question in developmental neurobiology is how the brain's diverse neuronal types arise from multipotential progenitors (Lodato \& Arlotta, 2015; McConnell, 1991; Wamsley \& Fishell, 2017). The vertebrate retina has been a valuable model for addressing this question: it is about as complicated as any other region of the brain, but has several features that facilitate mechanistic analysis (Dowling, 2012). The retina contains five classes of neurons - photoreceptors that sense light, three interneuronal classes (horizontal, bipolar and amacrine cells) that process visual information, and retinal ganglion cells (RGCs) that pass the information to the rest of the brain through the optic nerve (Fig. 1a) (Masland, 2012). These classes can be divided into numerous types, 130 in mouse and chick, each of which has characteristic morphological, physiological and molecular properties, and plays distinct roles in information processing (Baden et al., 2016; Franke et al., 2017; Goetz et al., 2021; Macosko et al., 2015; Rheaume et al., 2018; Shekhar et al., 2016; Shekhar \& Sanes, 2021; Tran et al., 2019; Yamagata, Yan, \& Sanes, 2021; Yan et al., 2020). Remarkably, nearly all types are distributed across the entire retina (Kay, Chu, \& Sanes, 2012; Keeley, Eglen, \& Reese, 2020; Rockhill, Euler, \& Masland, 2000), so morphogen gradients, which play a critical role in other parts of the central nervous system (e.g. (Sagner \& Briscoe, 2019)), cannot provide an explanation for retinal neuronal diversification (Marquardt \& Gruss,

Seminal studies have provided deep insights into how retinal classes arise(Bassett \& Wallace, 2012; Cepko, 2014). First, lineage tracing in rodents and frogs showed that single retinal progenitor cells (RPCs) can give rise to neurons of all classes as well as glia, and are therefore multipotential (Holt, Bertsch, Ellis, \& Harris, 1988; Turner \& Cepko, 1987; Turner, Snyder, \& Cepko, 1990; Wetts \& Fraser, 1988). Second, the competence of multipotential RPCs to generate cells of particular classes changes over time, accounting for their sequential (but overlapping) birth windows (Cepko, 2014; Livesey \& Cepko, 2001). Such segregation of birth windows is a hallmark of many neuronal systems (Holguera \& Desplan, 2018), and is believed to arise from the 
104 differential temporal regulation of gene expression in RPCs (Blackshaw et al., 2004; 105 Brown, Patel, Brzezinski, \& Glaser, 2001; S. Chen et al., 1997; Clark et al., 2019; 106 Trimarchi, Stadler, \& Cepko, 2008). Third, competence is probabilistic rather than 107 deterministic, with stochastic factors accounting for variations in the distribution of cell 108 classes generated by individual RPCs (Boije, MacDonald, \& Harris, 2014; Gomes et al., 109 2011; Johnston \& Desplan, 2010).

111 In contrast to these well-established tenets of neuronal class generation, we know far 112 less about how immature postmitotic neurons (which we call neuronal precursors here) 113 committed to a specific class identity diversify into distinct types. We address this issue 114 here, focusing on RGCs. All RGCs are similar in many respects: for example, they all 115 elaborate dendrites that receive input from amacrine and bipolar interneurons, send 116 axons through the optic nerve, and use glutamate as a neurotransmitter (Sanes \& 117 Masland, 2015). However, they differ in molecular, morphological and physiological 118 details, which have led to their division into 45 distinct types in mice (Baden et al., 119 2016; Bae et al., 2018; Goetz et al., 2021; Rheaume et al., 2018; Tran et al., 2019). 120 Most of these types appear to be feature detectors that collectively transmit a diverse 121 set of highly processed images of the visual world to the rest of the brain (Baden, Euler, 122 \& Berens, 2020; Sanes \& Masland, 2015). Several genes have been implicated in 123 maturation of a few mouse RGC types (Clark et al., 2019; Kiyama et al., 2019; Liu et al., 124 2018; Lo Giudice, Leleu, La Manno, \& Fabre, 2019; Lyu \& Mu, 2021; Peng et al., 2017; 125 Sajgo et al., 2017), but a comprehensive investigation of RGC diversification has been 126 lacking.

To gain insight into how and when adult RGC types emerge, we used high-throughput single-cell RNA-seq (scRNA-seq) to profile RGC precursors during embryonic and postnatal life in mice. We find that the number and distinctiveness of molecularly defined groups of precursors increases with developmental age, implying that types arise by a gradual process rather than from $\sim 45$ committed precursor types. Using statistical inference approaches, we identify fate associations among immature RGCs as transcriptomically distinct types emerge. These analyses suggest a model in which 
135 types arise from multipotential precursors by a process of restriction that we term fate decoupling. The decoupling is gradual and asynchronous, resulting in different types emerging at different times. We also use markers of RGCs that project to contralateral or ipsilateral retinorecipient areas to subdivide each type by its projection pattern, leading to the conclusion that laterality may be specified prior to type identity is fixed. Together, our results provide both a model of RGC diversification and a computational 141 framework that can be applied generally to analyze the diversification of closely related neuronal types within a class.

\section{Results}

145

\section{Transcriptomic atlas of developing mouse RGCs}

147 Mouse RGCs are born between approximately embryonic days (E) 11 and 17 with new148 born RGCs exiting the mitotic cycle near the apical margin, then migrating basally to 149 form the ganglion cell layer (Drager, 1985; Marcucci, Soares, \& Mason, 2019; Voinescu, 150 Kay, \& Sanes, 2009) (Fig. 1b). Reported birthdates differ among publications, and are 151 complicated by naturally occurring cell death and the central-peripheral developmental 152 gradient, but a detailed analysis concludes that $>95 \%$ of RGCs in adult mouse retina 153 are born after E12.8 and $>85 \%$ before E16 (Farah \& Easter, 2005). Shortly after they 154 are born, RGCs extend axons through the optic nerve, with some reaching 155 retinorecipient areas by E15 (Godement, Salaun, \& Imbert, 1984; Osterhout et al., 156 2011) and forming diverse projection patterns (Martersteck et al., 2017). During early 157 postnatal life, they extend dendrites apically into the inner plexiform layer of the retina, 158 receiving synapses from amacrine cells by postnatal day $(\mathrm{P}) 4$ and bipolar cells a few 159 days later (Kim, Zhang, Meister, \& Sanes, 2010; Lefebvre, Sanes, \& Kay, 2015). Light 160 responses are detected in RGCs by P10 but image-forming vision does not begin until 161 eye-opening, around P14 (Hooks \& Chen, 2020).

163 To determine when and how RGCs diversify, we used droplet-based scRNA-seq 164 (Macosko et al., 2015; Zheng et al., 2017) to profile them at 5 stages: E13 and E14 165 (during the period of peak RGC genesis), E16 (by which time RGCs axons are reaching 
166 target retinorecipient areas), P0 (as dendrite elaboration begins), and P5 (shortly after

167 RGCs begin to receive synapses). As RGCs comprise $\leq 1 \%$ of retinal cells (Jeon, 168 Strettoi, \& Masland, 1998), we enriched them with antibodies to two RGC-selective cell169 surface markers, Thy1/CD90 (Barres, Silverstein, Corey, \& Chun, 1988) and L1cam 170 (Demyanenko \& Maness, 2003) (Figure 1-figure supplement 1a).

172 We obtained a total of 98,452 single-cell transcriptomes with acceptable quality metrics 173 (Methods). Of these, we identified 75,115 (76\%) as RGCs based on their expression of 174 canonical RGC markers including Rbpms (an RNA-binding protein) and Slc17a6 (the 175 vesicular glutamate transporter VGLUT2) (Figs. 1c-e, Figure 1-figure supplement 1b176 c). Non-RGCs included amacrine cells (Tfap2a+Tfap2b+), cone photoreceptors $177(\mathrm{Otx} 2+\mathrm{Cr} x+)$, microglia $(P 2 r y 12+C 1 q a+)$, anterior segment cells $(M g p+B g n+)$, and 178 retinal progenitor cells (RPCs). Anterior segment cells were found only in E13 and E14 179 samples because whole eyes were dissociated at these stages. RPCs formed a continuum, containing both "primary" RPCs expressing cell-cycle related genes (e.g. Mki67, Ccnd5, Birc5) and previously described RPC regulators (e.g. Sfrp2, Vsx2 and Fgf15), and "neurogenic" RPCs expressing proneural transcription factors (e.g. Hes6, Ascl1, Neurog2)(Clark et al., 2019). Importantly, these markers were not expressed in cells annotated as RGCs (Figure 1-figure supplement 1d). These stringent criteria ensured that our dataset comprised postmitotic committed RGCs, allowing us to focus on their diversification and maturation.

Overall, we recovered $\sim 5,900$ to 18,500 RGCs at each of the five time points. Of the two surface markers used for enriching RGCs, Thy1 was effective at later stages as shown previously (Kay et al., 2011; Rheaume et al., 2018; Tran et al., 2019), whereas L1cam expression was more selective at E13 and E14 (Figure 1-figure supplement 1b,c). However, identical clusters were observed with both methods at E13, E14 and E16, albeit with different frequencies. This concordance supports the idea that neither marker

194 fails to capture particular RGC types. To further evaluate the effectiveness of our 195 enrichment strategy at early stages, we compared our data with two recent studies in 196 which developing retinal cells were profiled using scRNA-seq without any enrichment 
197 (Clark et al., 2019; Lo Giudice et al., 2019). A joint analysis of these datasets at 198 embryonic time points showed consistency in the transcriptional signatures of major cell 199 groups without discernible biases (Figure 1-figure supplement 1e-g). However, our 200 enrichment protocols increased the fractional yield of RGCs by $>3 X$ at E14 and E16 and 201 by $>100 \mathrm{X}$ at P0 (Figure 1-figure supplement 1h), which enabled us to resolve 202 heterogeneity within this class at immature stages. We also compared our P5 data with 203 those from an earlier study in which P5 RGCs were profiled (Rheaume et al., 2018) and 204 found a good correspondence (Figure 1-figure supplement 1i). For the analysis that 205 follows, we combined precursor RGCs (E13-P5) to a previously described dataset of 20635,699 mature RGCs at P56 (Tran et al., 2019). 


\section{Figure 1}
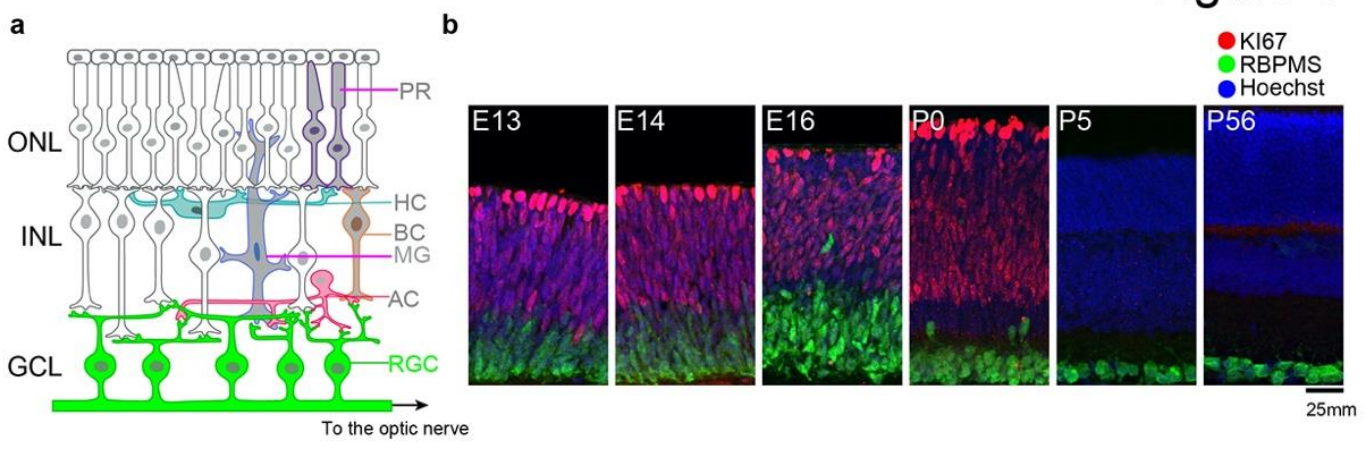

c
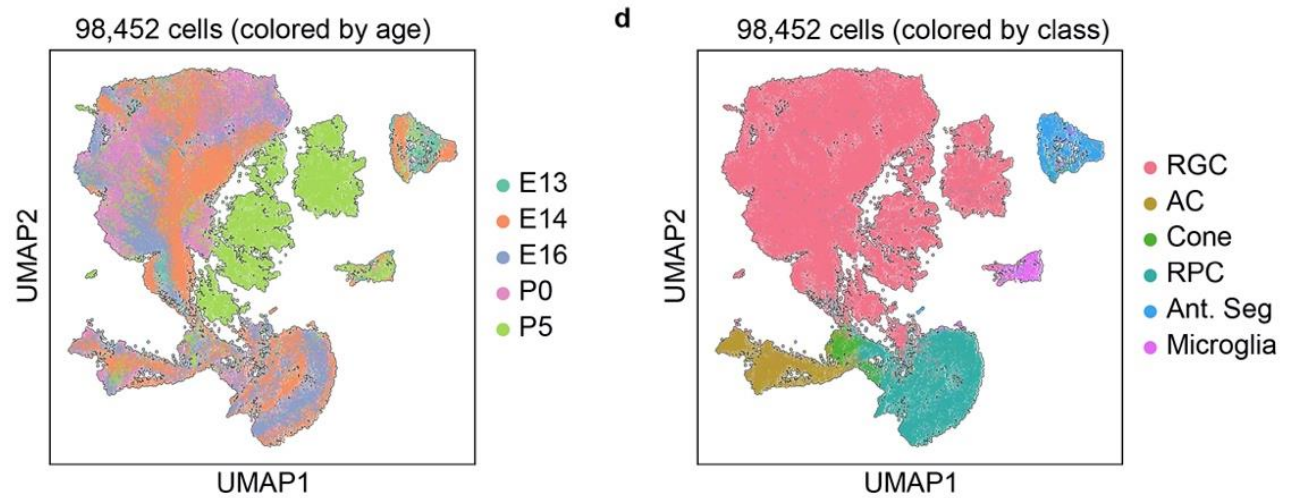

e

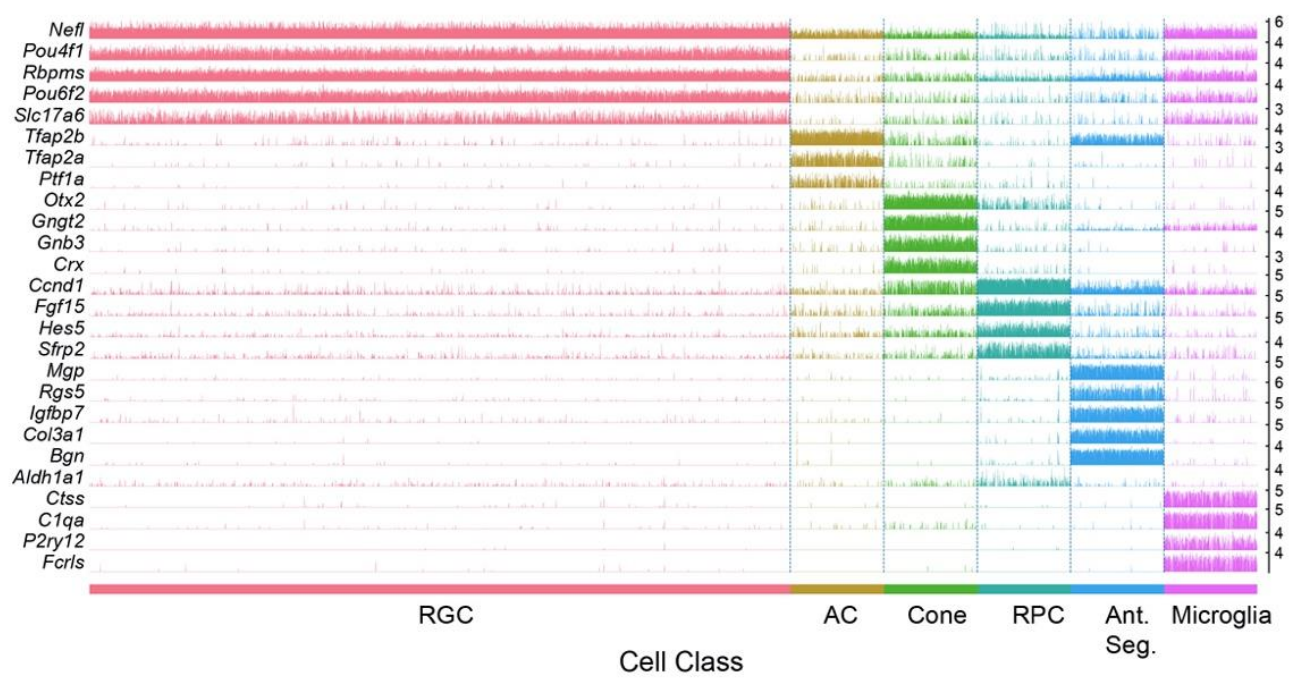

Figure 1, Transcriptomic profiling of single postmitotic RGCs during embryonic and postnatal development in mice.

210 a. Sketch of a section of the mouse retina showing major cell classes - photoreceptors (PRs; 211 rods and cones), horizontal cells (HCs), bipolar cells (BCs), amacrine cells (ACs), Müller glia 212 (MGs) and retinal ganglion cells (RGCs). PRs reside in the outer nuclear layer (ONL), while 213 BCs, HCs and most ACs reside in the inner nuclear layer (INL). RGCs and some ACs reside in 214 the ganglion cell layer (GCL). Axons of RGCs project to higher visual areas via the optic nerve. 
b. Retinal section of the indicated ages labeled for the cell-cycle marker MKI67 (red) and the RGC marker RBPMS (green); nuclei are counterstained by the Hoeschst dye (blue). 217 Micrographs are orientated as the schematic in panel a.

218 c. Visualization of transcriptional diversity of 98,452 cells using Uniform Manifold Approximation 219 and Projection (UMAP), a nonlinear dimensionality reduction algorithm that assigns proximal $x-y$ coordinates to cells (dots) with similar transcriptional profiles(Becht et al., 2019).

d. Same as c, with cells colored by cell class, assigned based on transcriptional signatures displayed in panel e. RPC, retinal progenitor cells; Ant. Seg., anterior segment cells.

e. Tracksplot showing expression patterns of cell-class specific marker genes (rows) across single cells (columns). Cells are grouped by class as in d. For each class, we randomly sampled $20 \%$ of total cells covering all immature time points (E13, E14, E16, P0, P5). For each gene, the scale on the $y$-axis (right) corresponds to normalized, log-transformed transcript counts detected in each cell.

\section{Immature RGCs diversify postmitotically}

230 One can envision two extreme models of RGC diversification. In one, RGC type would 231 be specified at or before mitotic exit, with each type arising from a distinct set of 232 committed precursors. At the other extreme, all precursor RGCs would be identical 233 when they exit mitosis, and gradually acquire distinct identities as they mature (Fig. 2a). 234 Intermediate models could involve multiple groups of precursor RGCs, each biased 235 towards a distinct set of terminal types.

237 To distinguish among these alternatives, we analyzed the transcriptomic diversity of 238 RGCs at each developmental stage using the same dimensionality reduction and graph 239 clustering approaches devised for analysis of adult RGCs (Tran et al., 2019) (see 240 Methods). This analysis led to three main results.

242 First, RGCs were already heterogeneous soon after mitotic exit. There were 10 243 transcriptionally defined precursor clusters at E13 (Fig. 2b), before or at the peak time 244 of RGC birth. The number of discrete clusters increased only slightly by E14 (from 10 to 245 12; Fig. 2c), arguing against a model in which the number of precursor types 246 extrapolated back to one. No single cluster dominated the frequency distribution at 247 either time, as would be expected if a totipotent precursor RGC were to exist shortly 248 after terminal mitosis. 
250 Second, the number of transcriptionally defined clusters increased gradually, between 251 E13 and adulthood, reaching 45 only after P5 (Figs. 2b-g). Several arguments indicate 252 that this increase is biologically significant rather than being an artifact of the data or 253 computational analysis. (1) We used the same clustering procedure at all ages. (2) The 254 qualitative trends were robust against variations in clustering parameters. (3) All 255 embryonic clusters contained cells isolated with both cell markers, L1cam and Thy1 256 (Figure 2-figure supplement 1a-c), indicating that lower cluster numbers at early 257 stages did not result from biased collection methods. (4) The increase in the number of 258 effective molecular types was robust as demonstrated by three diversity indices - Rao, 259 Simpson and Shannon - all of which buffer against artificial inflation of diversity due to 260 small clusters (Fig. 2h, Figure 2-figure supplement 1d; see Methods). (5) There was 261 no systematic dependence of the number of clusters on the number of cells. For 262 example, we identified 12 clusters from 17,100 cells at E14 and 38 clusters from 17,386 263 cells at P5.

264

Third, the transcriptomic variation became increasingly discrete with age. We quantified this increase in inter-cluster separation by calculating (1) the average cross-validation error of a multi-class classifier, and (2) the ratio of mean cluster diameter to mean intercluster distance in the low dimensional embedding (Methods). Both metrics decrease in numerical value as the clusters are more-well defined. From these trends, we conclude that the boundaries between RGC clusters become sharper as development proceeds (Figs. 2i,j).

Taken together, our results show that transcriptomic clusters of RGCs increase in 275 specified at the progenitor stage. 
Figure 2
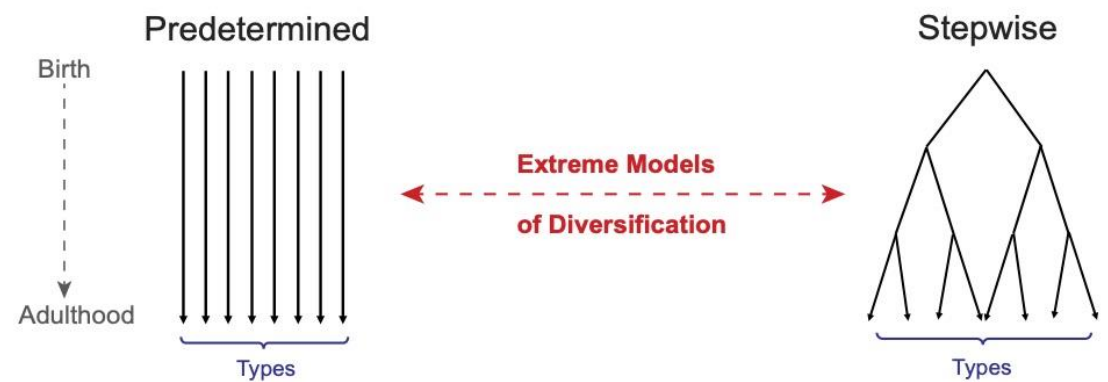

b

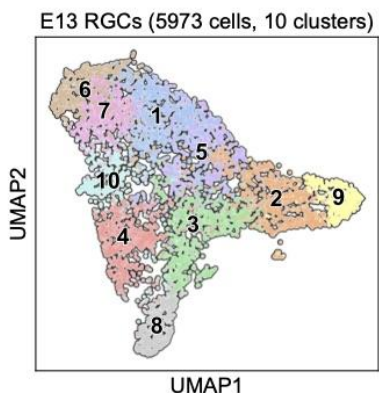

e

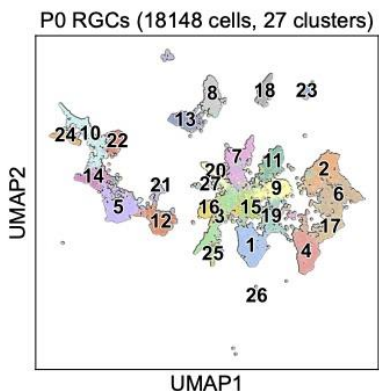

h

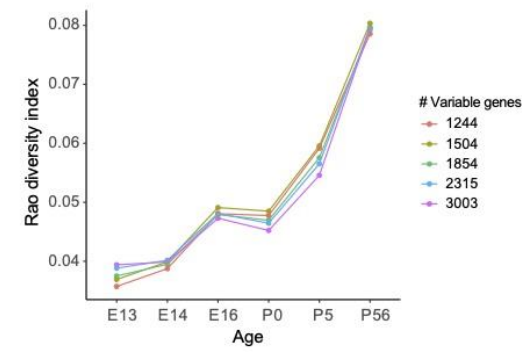

C

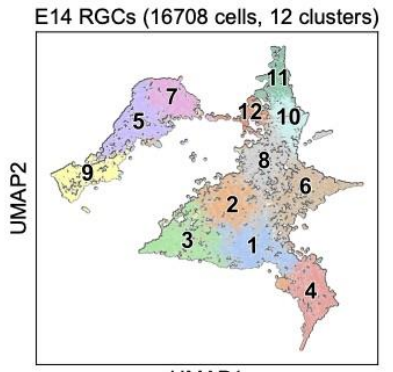

UMAP1

f
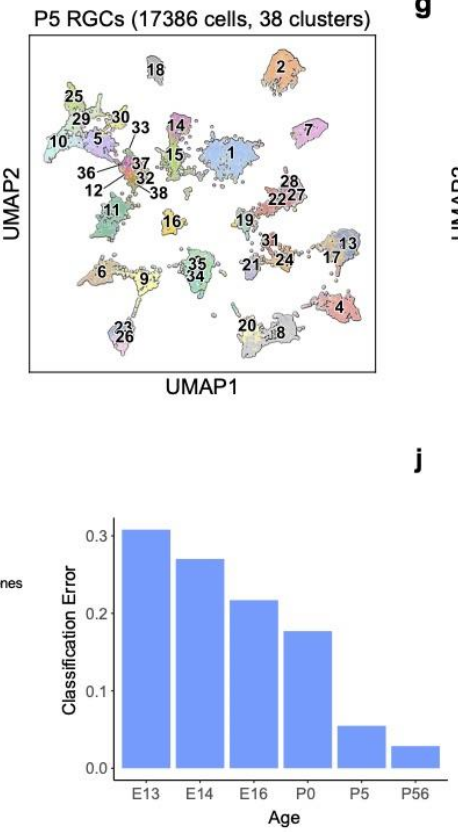

j d
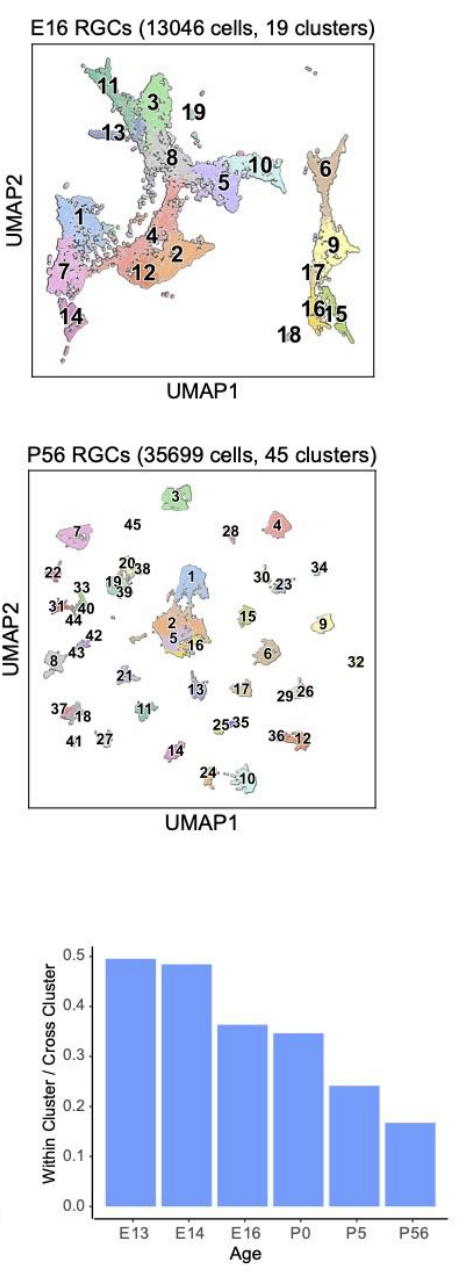

Figure 2. The number and discreteness of transcriptomic clusters of RGCs increases with age. of the terminal types by the time of birth (i.e. mitotic exit) or shortly after. Alternatively (right), process of restriction. 
b-g. Visualization of transcriptomic diversity of immature RGCs at E13 (b), E14 (c), E16 (d), P0 (e), P5 (f) and P56 (g) using UMAP. Cells are colored by their cluster identity, determined independently using dimensionality reduction and graph clustering (Methods). Clusters are numbered based on decreasing size at each age. Data for adults (P56) are replotted from (Tran et al., 2019). In that study 45 transcriptomic types were identified via unsupervised approaches, one of which was mapped to 2 known functional types by supervised approaches. We do not distinguish them in this study.

293 h. Transcriptional diversity of RGCs as measured by the Rao diversity index (y-axis) increases 294 with age (x-axis). The trend is insensitive to the number of genes used to compute inter-cluster distance (colors). See Methods for details underlying the calculation.

i. Transcriptomic distinctions between RGC clusters become sharper with age as shown by decreasing average per-cluster error of a multiclass-classifier with age. Gradient boosted decision trees(T. Chen \& Guestrin, 2016) were trained on a subset of the data, and applied on held out samples to determine the test error.

j. RGC clusters also become better separated in the UMAP embedding, as shown by decreasing values of the average relative cluster diameter with age.

\section{Temporal relationships among immature RGC clusters}

We next investigated the temporal relationships among precursor RGC clusters identified at different ages. We again consider two extreme models. In a "specified" model, each terminal type arises from a single cluster at every preceding developmental stage (Fig. 3a, left). In this model, distinct transcriptomic states among precursor RGCs correspond to distinct groups of fates. At the other extreme, distinct clusters would share similar sets of fates (Fig. 3a, right). In an intermediate model, fates of precursor clusters would exhibit partial overlap.

As a first step in discriminating among these scenarios, we used transcriptome-wide correspondence among clusters as a proxy for fate association. We identified mappings between clusters across each pair of consecutive developmental stages (E13-E14, E14E16, E16-P0, P0-P5, and P5-P56) using gradient boosted trees (T. Chen \& Guestrin, 2016), a supervised classification approach (Methods). In each case, a classifier trained on transcriptional clusters at the older stage was used to assign older cluster labels to cells at the younger stage (e.g. E16 labels assigned to E14 RGCs). Patterns expected for the extreme models are schematized as "confusion matrices"(Stehman, 1997) in the lower panels of Fig. 3a.

Correspondence fell between the two extremes (Figs. 3d-h and Figure 3-figure supplement 1a-d). We quantified the extent of correspondence using two metrics: 
324 Normalized Conditional Entropy (NCE) and the Adjusted Rand Index (ARI) (Methods). 325 Both NCE and ARI are restricted to the range $(0,1)$, with lower values of NCE and 326 higher values of ARI consistent with a specified mode of diversification. Both metrics 327 exhibited an increased degree of specificity with age (Figs. 3b,c). Since NCE and ARI 328 provide a single measure of specificity for the entire datasets being compared, we also 329 computed a "local metric", the Occupancy Fraction, which quantifies mapping specificity 330 for each cluster (Methods). Results based on this metric were consistent with increased 331 specificity of correspondence with age (Figure 3-figure supplement 1e). Overall, this 332 analysis of transcriptomic correspondence suggests that poorly specified relationships 333 among transcriptomic clusters at early stages are gradually refined to yield increasingly 334 specific associations at later stages. 
Figure 3
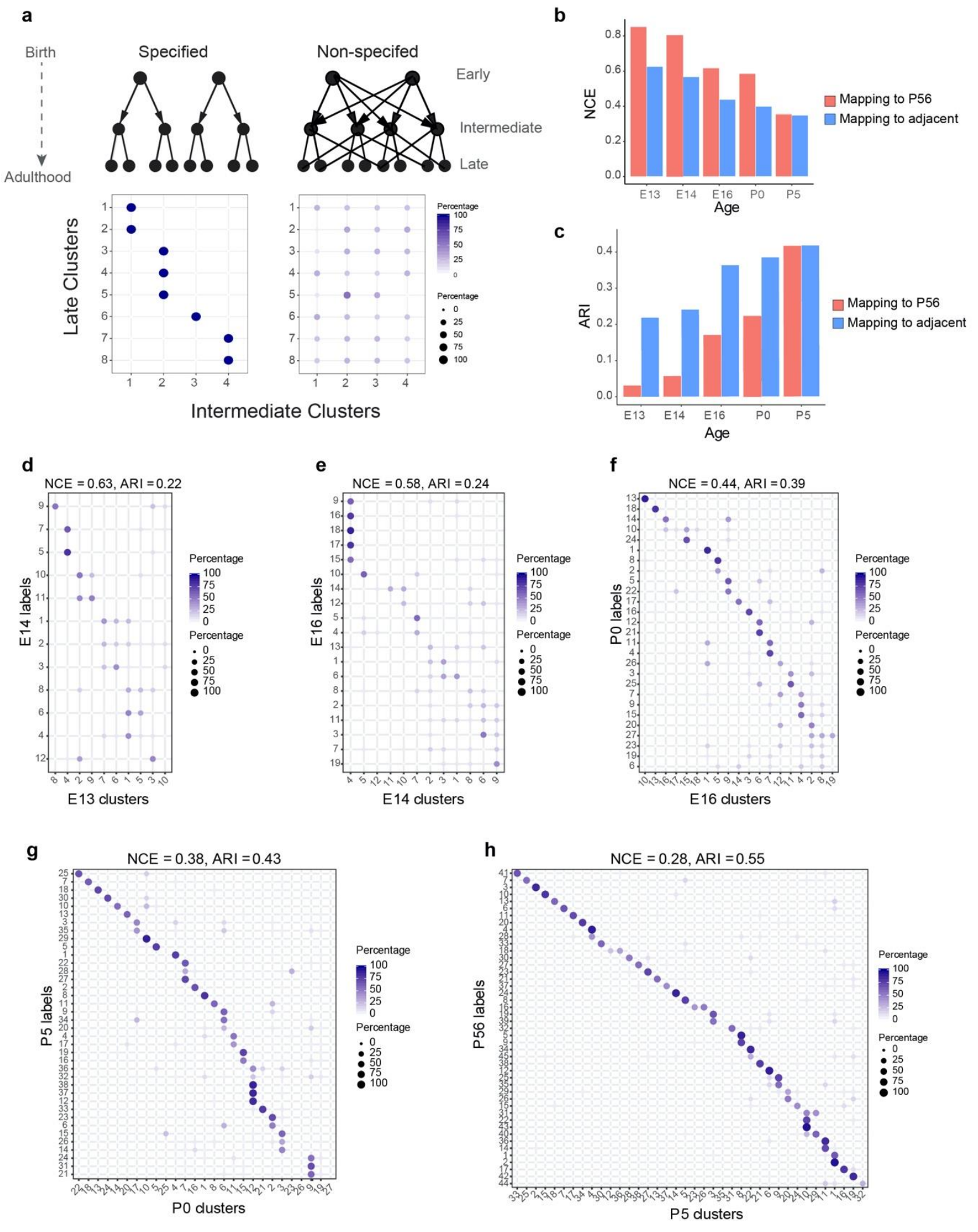

336 Figure 3. Incompletely specified temporal relationships among RGC clusters. transcriptomic clusters of immature RGCs, and arrows denote fate relationships. Bottom: 
Confusion matrices depicting transcriptomic correspondence between late and early clusters expected for the two modes. Circles and colors indicate the percentage of a given late cluster (row) assigned to a corresponding early cluster (column) by transcriptome-based classifier trained on early clusters. The number of late and early clusters have been set to eight and four for illustration purposes.

b. Barplot showing values of the normalized conditional entropy (NCE) for each age calculated using the transcriptional cluster IDs and the Xgboost-assigned cluster IDs corresponding to the next age or to P56 (E.g. for E13, the NCE was calculated across E13 RGCs by comparing their transcriptional cluster ID with assigned E14 cluster IDs based on a classifier trained on the E14 data). Lower values indicate specific mappings.

c. Same as b, but plotting values of the adjusted Rand Index (ARI), where larger values correspond to higher specificity.

d-h. Confusion matrices (representation as in a), showing transcriptomic correspondence between consecutive ages: E14-E13 (d), E16-E14 (e), P0-E16 (f), P5-P0 (g), P56-P5 (h). In each case, the classifier was trained on the late time point and applied to the early time point. Rows sum to $100 \%$.

\section{Immature RGCs are multipotential}

357 The classification analysis presented so far relied on comparing clusters between ages and was therefore unable to link individual precursors to specific terminal fates. At one extreme, individual precursor clusters might contain several groups of cells, each committed to a distinct, small number of fates. Alternatively, individual cells might be as multipotential as the clusters in which they reside (Fig. 4a).

Unfortunately, this approach does not afford a straightforward way to explore variations in patterns of fate associations within clusters. We therefore turned to WaddingtonOptimal Transport (WOT), a computational method rooted in optimal transport theory (Kantorovich, 1942; Monge, 1781) that utilizes scRNA-seq measurements at multiple stages to infer developmental relationships (Schiebinger et al., 2019). Briefly, WOT computes a "transport matrix" $\Pi$ between each pair of consecutive ages with elements $\Pi_{i j}$ encoding fate associations between a single RGC $i$ at the younger age and RGC $j$ at the older age (see Methods). WOT directly computes fate associations at the level of individual cells without requiring clustering as a prior step. We conducted extensive 372 computational tests to assess the numerical stability of associations reported by WOT 373 (Figure 4-figure supplement 1). We also determined that when collapsed to the level 374 of clusters, the WOT inferred transport maps strikingly mirrored the confusion matrices 375 obtained from multi-class classification (Figure 4-figure supplement 2). 
376 Based on the success of these tests, we applied WOT to compute the "terminal fate" for each precursor RGC. We leveraged the fact that in WOT, fate associations between RGCs at non-consecutive ages (e.g. E16 and P56) can be estimated in a principled way 379 by multiplying the intermediate transport matrices. This yielded a fate vector $\vec{f}$ for each 380 of the 75,115 immature RGCs, whose kth element $f_{k}$ represents the predicted probability of commitment to adult type $k \in(1,2, \ldots, 45)$ (Methods). A fully committed precursor would have all but one element of $\vec{f}$ equal to zero, whereas a partially committed precursor would have multiple non-zero elements in $\vec{f}$. Since the elements of $\vec{f}$ are interpreted as probabilities, they are normalized such that $\sum_{\mathrm{k}} f_{k}=1$.

We quantified the commitment of each precursor by computing its "potential" $P=\frac{1}{\sum_{\mathrm{k}} f_{k}^{2}}$, which is defined analogously to the "inverse participation ratio" in physics (Fyodorov \& Mirlin, 1992). In our case, the value of $P$ for a given RGC ranges continuously between 1 and 45, with lower values implying a commitment to specific fates, and higher values reflecting indeterminacy. Importantly, this measure of commitment does not rely on arbitrary thresholding of the $f_{k}$ values to assign precursors to types.

Five results emerged from this analysis.

- Nearly all prenatal RGCs (i.e. on or before P0) were multipotential rather than committed to a single terminal fate, with individual potentials distributed across a range of values (Fig. 4b).

- Multipotentiality was a general feature of immature RGCs, being present in cells of all clusters at E13, E14 and E16 (Figs. 4c-f).

- At early stages the average value of $P$ varied among transcriptomic clusters, reflecting asynchronous specification (Fig. 4c). The tempo of commitment is further explored in the next section.

- Although they were multipotential, no precursor RGC was totipotential (i.e. completely unspecified, corresponding to $P=45$ ). At E13 the average value of $P$ was $11.6 \pm 4.9$ which was 4 -fold lower than the maximum possible value of 45 , and no precursor had $P>30$. 
415 From these results, we conclude that early postmitotic RGCs are multipotential but not 416 totipotential, and that type identity is specified gradually via progressive restriction.

- Finally, inferred multipotentiality decreased gradually during development, and some persisted postnatally, (average $P=3.4 \pm 2.1$ at $\mathrm{P} 0$, and $1.6 \pm 0.9$ at P5; Figs. 4g,h).
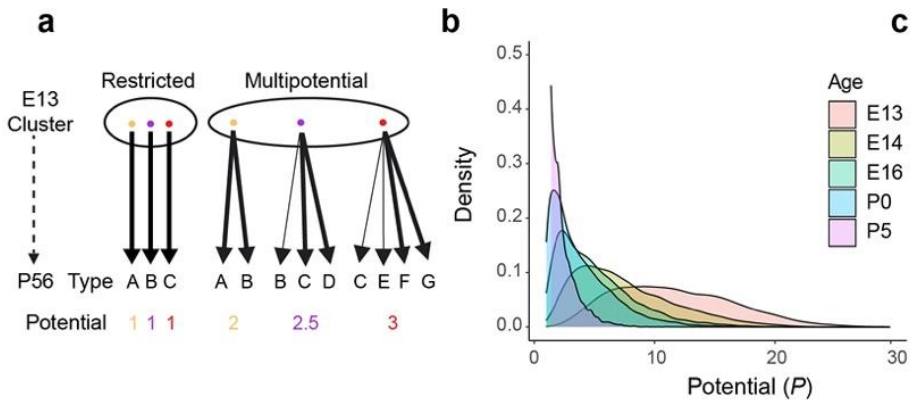

Figure 4

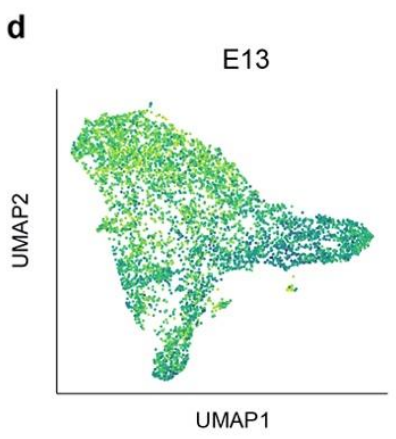

e
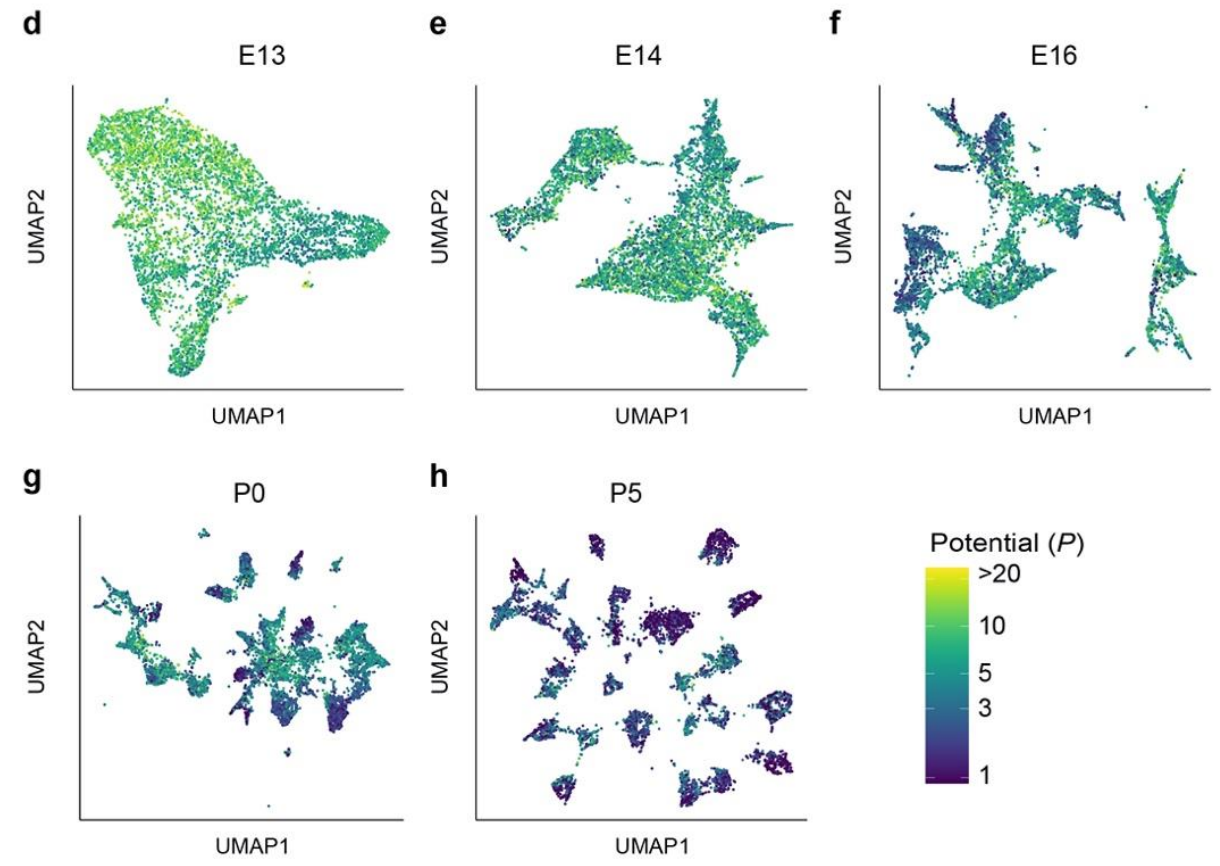

Figure 4. Multipotential fate associations between immature RGCs and terminal types inferred via Optimal Transport

a. Extreme models of diversification at single-cell resolution. Multipotential fate associations in a transcriptionally defined cluster (ellipse) could arise from a mixture of unipotential RGCs (left) or from multipotential RGCs (right). 
b. Distributions of potential $P$ across immature RGCs by age showing that restriction increases with age.

c. Inter- and intra-cluster variation of potential by age. At each age, variation in the potential values are shown for each transcriptomically defined cluster at that age. Dots denote the average potential and dotted lines depict the standard deviation for cells within each cluster. d-h. UMAP projections of E13 (d), E14 (e), E16 (f), P0 (g) and P5 (h) RGCs as in Fig. 2, but with individual cells colored by their inferred potential. Potential of all RGCs at P56 $=1$. The colorbar on the lower right is common to all panels, and values are thresholded at $P=20$.

\section{Asynchronous specification of mouse RGC types via fate decoupling}

As a first step in understanding the progressive restriction of RGC fate, we analyzed the extent to which pairs of mature types were likely to have arisen from the same set of immature precursors. To this end we computed a "fate coupling" value $C(l, m ; a g e)$ for each pair of terminal RGC types $(I$ and $m$ ), defined as the Pearson correlation coefficient between the values of $f_{l}$ and $f_{m}$ across all precursors at a given age (Methods). $f_{l}$ and $f_{m}$ are fate probabilities corresponding to types $/$ and $m$ as defined in 440 the previous section. Values of $C(l, m ; a g e)$ in our data ranged from -0.11 to 0.95 . 441 Higher values of $C(l, m ; a g e)$ indicate strong fate coupling between types $I$ and $m$, 442 implying the existence of common postmitotic precursors, whereas low $C(l, m ;$ age $)$ 443 values suggest that types $/$ and $m$ arose from largely nonoverlapping sets of precursors. 444 We visualized the pattern of fate couplings as network graphs, where the nodes 445 represent types and the edge weights represent values of $C(l, m ; a g e)$. The 446 arrangement of nodes was determined at E13 using a force directed layout algorithm 447 (Fruchterman \& Reingold, 1991), with pairwise distances being inversely proportional to 448 values of $C(l, m ; E 13)$, the fate coupling values at E13 (Fig. 5a). To visualize the 449 temporal evolution of these fate couplings, we retained the same layout of nodes while 450 updating edge weights according to $C(l, m$; age) (Figs. 5b-e).

452 Types that were coupled in fate at the earliest time point gradually decoupled as 453 development proceed. For example, at E13, 118/990 pairs (12\%) were strongly coupled 454 (threshold of $C(l, m ; a g e)>0.2$ as determined by randomization tests; see Methods), 455 while at P5, only 8/990 (<1\%) passed this criterion (Figs. 5a,e). Lowering this threshold 456 for coupling to 0.05 increased the number of strongly coupled pairs at P5 to only $2 \%$ 457 (20/990). 
459 Different pairs of types decoupled at different rates (Fig. 5f). As they decoupled, RGC 460 precursors became increasingly restricted to a single type (i.e. $f_{k} \gg f_{l \neq k}$ for a precursor 461 favoring type $k$ ). This corresponded to a "localization" of precursors in transcriptomic 462 space, and is a proxy for specification (see Methods). We modeled the extent of 463 localization vs. age via a logistic function (Fig. $\mathbf{5 g}$ and Figure 5-figure supplement 464 1d), and used this to calculate a specification time for each type $\left(\tau_{s p}\right)$ (see Methods for 465 details). Based on this analysis, $7 / 45$ types are specified postnatally. The average $\tau_{s p}$ 466 for RGCs was E17.8, but individual RGC types exhibited a wide range from E13.9 to 467 P5.2 (Fig. 5h). The inferred specification time was not correlated to adult frequency 468 (Fig. 5i).

470 We illustrate this range by considering three pairs of RGC types in Figure 5-figure 471 supplement 1. C12 and C22 (numbered as in Tran et al., 2019; see Fig. 2g) exhibit low 472 fate coupling at all ages profiled (Figure 5-figure supplement 1a), indicative of 473 separate precursor populations. In contrast, C19 and C20 decouple only at P0, implying 474 the existence of a common precursor throughout embryogenesis (Figure 5-figure 475 supplement 1b). C21 and C34 display an intermediate pattern, decoupling around E16 476 (Figure 5-figure supplement 1c). Taken together, these results suggest that RGC 477 types emerge by asynchronous fate decoupling of multipotential precursors. 


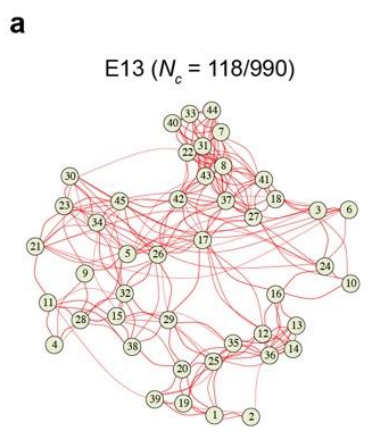

b

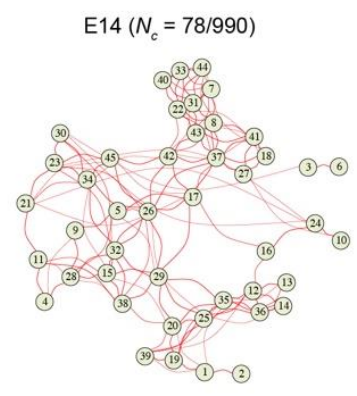

d

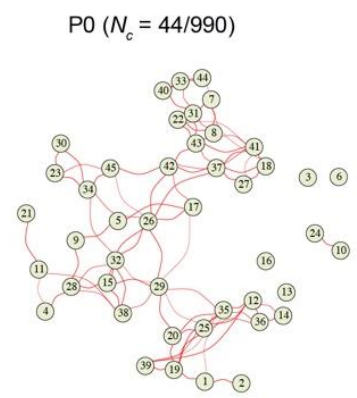

e

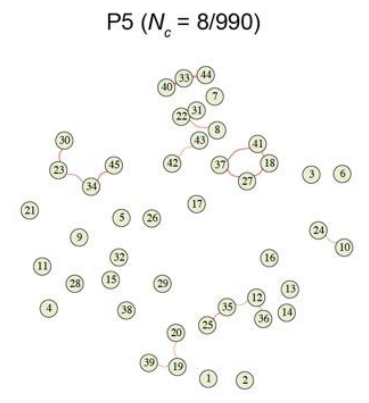

Figure 5
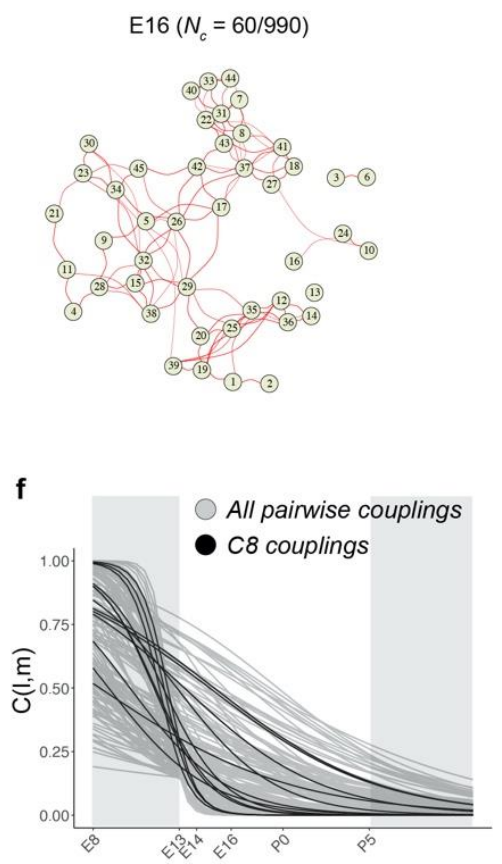

479

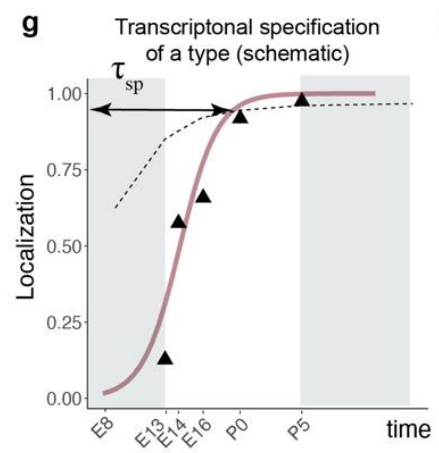

h

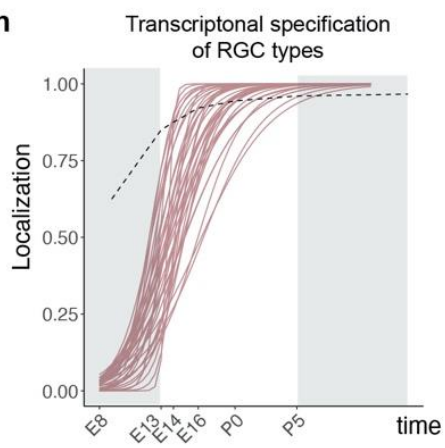

i

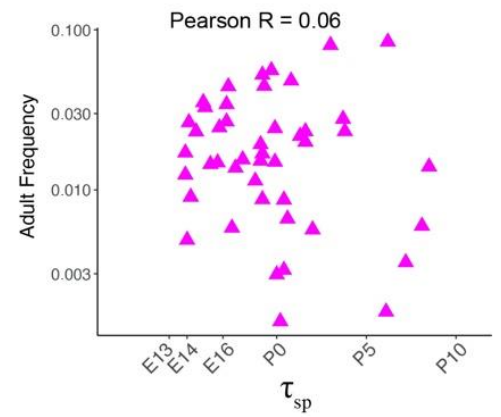

480

481

482

483

484

485

486

487

488

489

490

491

492

\section{Figure 5. Fate decoupling of RGC types}

a. Force-directed layout visualization of fate couplings at E13, with nodes representing RGC types (numbered as in Tran, 2019) and the thickness of edges representing values of $C(I, m ; E 13)$. Edges with $C(I, m ; E 13)<0.2$ are not shown. Number of edges with $C(I, m ; E 13)>$ 0.2 are indicated on top.

b-e. Visualization of fate couplings at E14 (B), E16 (C), P0 (D) and P5 (E). The positions of the nodes are maintained as in panel a, but the edges are redrawn based on values of $C(I, m$;age) at each age. As in panel a, we only show edges $C(I, m$; age $)>0.2$.

$f$. The decay of pairwise fate couplings (y-axis) with age (x-axis). Each line corresponds shows the temporal decay of $C(I, m)$ for RGC pair I and m estimated via a logistic model (Methods). For each pair, couplings at each age were fit to a model $C(l, m ;$ age $)=1 /\left(1+e^{\beta_{0}+\beta_{1} * a g e}\right)$ with $\beta_{0}, \beta_{1}$ representing fitted parameters. The fitting was performed using data for ages E13, E14, E16, P0 and P5. The shaded portions correspond to the periods E8-E13 and P5- represent 
extrapolations of the model. Black lines highlight the decay of all non-zero pairwise couplings for RGC type $C 8$ as an example. g. Schematic showing logistic modeling to estimate specification time $\tau_{s p}$ for a particular type.

The $y$-axis is a measure of the extent to which precursors biased towards the type are present in a single transcriptomically defined cluster (i.e. localization, see Methods for details). Localization is defined as a numerical value in the range $(0,1)$ with higher values consistent with increasing specification. Individual triangles represent the localization values computed using WOT inferred fate couplings at each age, while the curve represents the fit using the logistic model. Dotted line shows the minimum threshold a type to be specified at each age. Its curved shape arises due to the increase in the number of clusters with age.

h. Localization curves (as in panel g) for the 38 RGC types showing the range of inferred specification times. 7 low frequency types have been excluded from display (see Figure 5figure supplement $1 \mathrm{~d}$ ).

i. Scatter plot showing poor correlation between adult frequency of a type (from (Tran et al., 2019)) and its predicated specification time (calculated from h).

\section{Fate decoupled groups of RGC types defined by transcription factors}

Because fate coupling is a metric of inferred overlap of developmental history, it is likely that tightly coupled types share common precursors. This relationship implies that tightly coupled types might also be specified by common transcriptional programs. As a step towards identifying candidate fate determinants, we identified 8 transcription factors (TFs) that are expressed by distinct groups of mature RGC types (Fig.6a, Figure 6figure supplement 1a). Three of these are well-characterized RGC-selective TFs: Foxp2, expressed by 5 F-RGC types (Rousso et al., 2016); Tbr1, expressed by 5 TRGC types (Liu et al., 2018); and Eomes (also known as Tbr2), expressed by 7 types (C.-A. Mao et al., 2020; Tran et al., 2019). The 7 Eomes/Tbr2 types include the melanopsin expressing intrinsically photosensitive (ip) RGC types (Berson, Dunn, \& Takao, 2002). The remaining five were Neurod2, Irx3, Mafb, Tfap2d, and Bnc2 that label 8, 5, 4, 6, and 3 types respectively. Eomes types also co-expressed Tbx20 and Dmrbt1 while Neurod2 types also co-express Satb2. Together, 40/45 mature types expressed at least one of these TFs in a manner that was, with few exceptions, mutually exclusive. In many cases, the fate proximity of types that shared TF expression was

527 We refer to these TF-based groups as fate-restricted RGC subclasses - an 528 intermediate taxonomic level between class and type based on inferred fate 529 relationships. Consistent with their definition, the pairwise fate coupling among types 
530 from different subclasses was significantly lower than among types from the same

531 subclass (Fig. 6b). Thus, precursor RGC states associated with any two subclasses are

532 more distinct than those associated with any two types. This is evident by the significant

533 separation at E13 and negligible overlap at P5 for precursors favoring the Eomes, Mafb

534 and Neurod2 subclasses respectively, as shown in Fig. 6c-e.

536 We also asked whether the TF-based subclasses differed in inferred transcriptomic 537 specification time $\tau_{s p}$, as defined in Fig. 5g. As shown in Figs. 6f-h, and Figure 6-

538 figure supplement $\mathbf{1 b}$-e, four subclasses were specified within a narrow interval 539 (E16.8-E17.2), but three others differed substantially. The average specification time for 540 the Eomes group was E14.6 ( $p<0.0001$, Student's t-test, compared to the mean for all 541 types), while that for the Mafb and Neurod2 groups were E16.9 $(p<0.001)$ and E18.5 ( $p$ $542<0.0001)$, respectively. The early specification of the Eomes group is consistent with 543 birthdating studies showing the average earlier birthdate of ipRGCs compared to all 544 RGCs (McNeill et al., 2011).

546 In summary, our results suggest the existence of fate-restricted RGC subclasses that 547 arise from distinct sets of precursors and diversify into individual types. This method of 548 defining RGC groups, which relies on inferred proximity of precursors in transcriptomic 549 space, is distinct from previous definitions of RGC subclass based on shared patterns of 550 adult morphology, physiology or gene expression (see Discussion). Accordingly, the 551 fate couplings at E13 were only weakly correlated with transcriptomic proximity in the 552 adult retina (Figure 6-figure supplement 1f). Further, while TF-based groups align with 553 some previously defined subclasses (e.g. ipRGCs or Tbr1+RGCs), they do not map to 554 others subclasses such as alpha-RGCs (4 types) or T5-RGCs (9 types) (Figure 6555 figure supplement $\mathbf{1 g}$ ). 
a

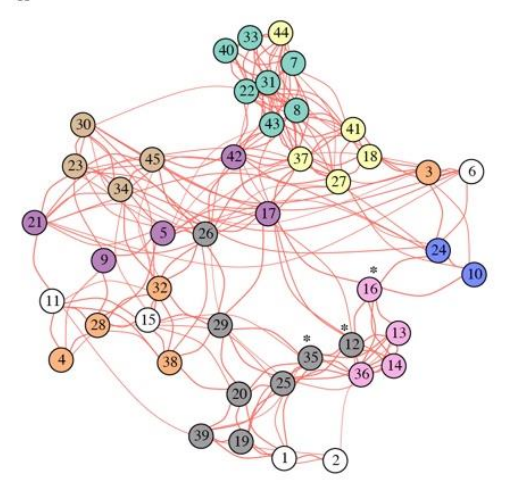

b

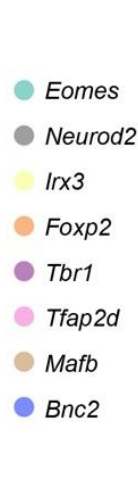

Figure 6

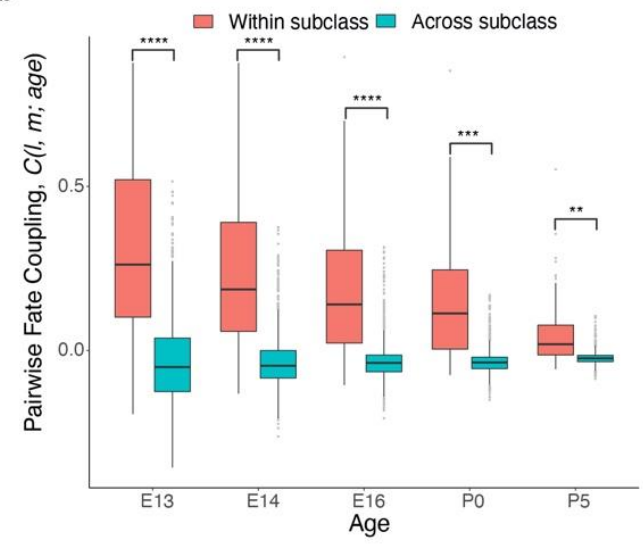

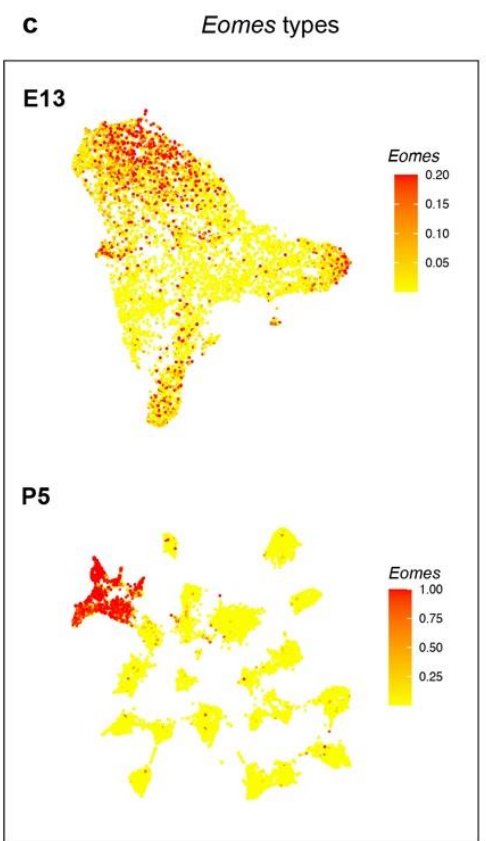

d

Mafb types

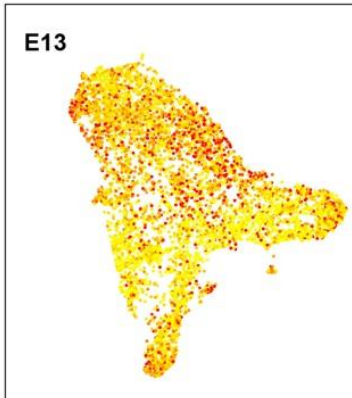

P5
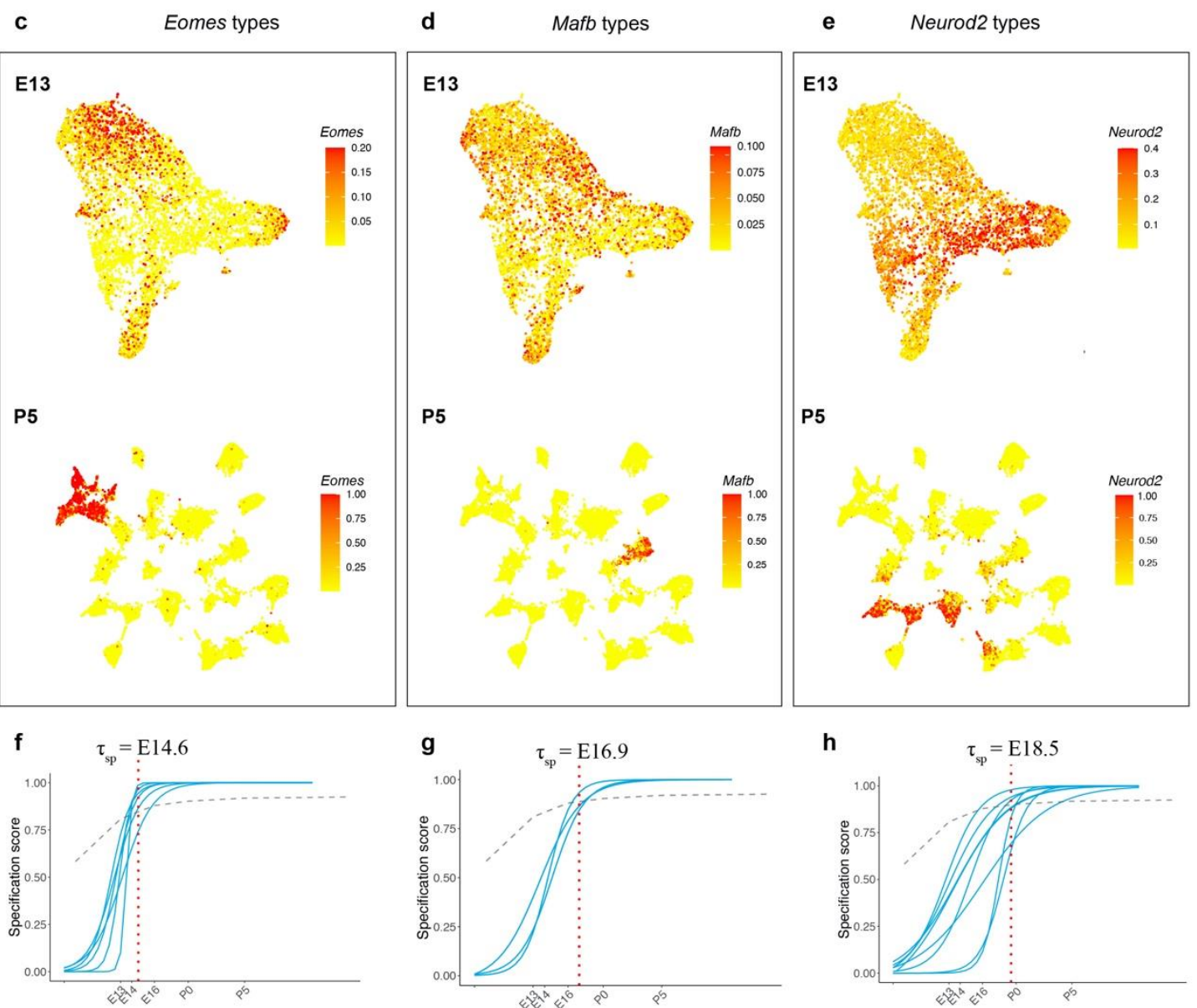

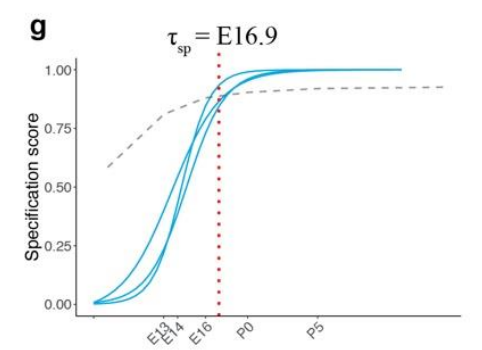

h

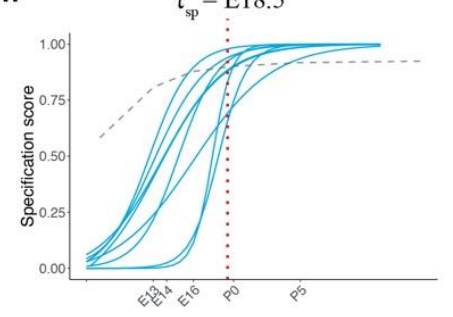

Figure 6. Temporal dynamics of RGC subsets expressing specific TFs

a. E13 network graph of fate couplings from Fig. 5a, with RGC types colored based on their selective expression of TFs at P56. Asterisks denote 3/45 types that express more than 1 TF (also see Figure 6-figure supplement 1a).

b. Box-and-whisker plots showing that pairwise fate couplings are higher between types within the same TF subclass than between types in different TF subclasses at all immature ages. Black horizontal line, median; bars, interquartile range; vertical lines, $1^{\text {st }}$ and $99^{\text {th }}$ percentile; 
565

dots, outliers. Stars indicate significant $\mathrm{p}$-values based on a two-sided t-test ${ }^{* * * *}, \mathrm{p}<10^{-7} ;{ }^{* * *}, \mathrm{p}$ $<10^{-5} ; * *, p<10^{-2}$ ).

c. Eomes+ types. Top: UMAP representation of E13 RGCs with cells colored based on their cumulative fate association towards the 7 Eomes+ types. Bottom: UMAP representation of P5 RGCs with cells colored based on their cumulative fate association towards the 7 Eomes+ types. The value corresponding to the color of each cell (colorbar, right) can be interpreted as the probability of commitment towards the corresponding subclass.

d. Same as c for Mafb+ types

e. Same as c for Neurod2+ types

f-h. Localization curves (as in Fig. 5g) for Eomes+ types (f), Mafb+ types (g) and Neurod2+ types (h). The mean inferred specification time $\tau_{s p}$ for each group is indicated on top of each panel.

\section{Transcriptomic profiles of ipsilateral and contralateral RGCs}

Finally, we considered the origin of two RGC groups defined by their projections: those with axons that remain ipsilateral at the optic chiasm (I-RGCs) and those that cross the midline to innervate contralateral brain structures (C-RGCs). The proportion of I-RGCs varies among vertebrates, in rough correspondence to the extent of binocular vision, ranging from none in most lower vertebrates to $~ 50 \%$ in primates. In mice, $3-5 \%$ of RGC axons remain ipsilateral, with most I-RGCs residing in the ventrotemporal (VT) retinal crescent (Mason \& Slavi, 2020). While some I-RGCs have been observed to project from the dorsocentral retina during embryonic stages, these are rapidly eliminated socalled "transient" I-RGCs (Soares \& Mason, 2015). Thus, in adulthood, C-RGCs are present throughout the retina while "permanent" I-RGCs are confined to the VT crescent.

The zinc-finger transcription factor Zic2 is expressed in a subset of postmitotic RGCs in VT retina, and is both necessary and sufficient for establishing their ipsilateral identity (Herrera et al., 2003); transient dorsolateral I-RGCs do not express Zic2 (Pak, Hindges, Lim, Pfaff, \& O'Leary, 2004). Is/2 marks a subset of C-RGCs throughout the retina and appears to specify a contralateral identity in part by repressing Zic2 (Pak et al., 2004). These two transcription factors were expressed in a mutually exclusive fashion in RGC precursors between E13 and E16 (Fig. 7a); Zic2 was down-regulated at later ages (Figure 7-figure supplement 1a). Furthermore, Zic2 expression at E13 correlated with Igfbp5 and Zic1, and anti-correlated with Igf1 and Fgf12, consistent with recent reports 
600 (Wang, Marcucci, Cerullo, \& Mason, 2016) (Fig. 7b and Figure 7-figure supplement 601 1b,c). We scored each cell at E13 based on its expression of ipsilateral genes 602 (Methods), confirming that expression of ipsilateral and contralateral gene signatures 603 were anticorrelated (Fig. 7c). Together, these observations support the idea that at E13 604 Zic2+ cells represent I-RGCs, and IsI2+ cells represent some but not all C-RGCs.

Using WOT, we then identified the descendants of presumptive I-RGCs at later ages. We found that I-RGCs comprised $4.3 \%$ of adult RGCs, consistent with the range of 3 $5 \%$ noted above. We queried these cells to identify genes that distinguished putative IRGCs and C-RGCs throughout the developmental time course. At a fold-change of $\geq 1.5$ we found 59 DE genes at E13 and 89 at E14 (Figs. 7e,f). In addition to Zic2, Igfbp5, Isl2 and Igf1, which had been used to define I-RGCs and C-RGCs at E13, they included Igfbpl1, Pou3f1 and Cntn2 enriched in I-RGCs, and Lmo2, Pcsk1n and Syt4 enriched in C-RGCs. The number of genes DE between I- and C-RGCs decreased after E14, with 20, 9 and 0 significant genes at E16, P0 and P5, respectively (Figure 7-figure supplement $1 \mathbf{d}, \mathbf{e})$, presumably reflecting the downregulation of axon guidance programs once retinorecipient targets have been reached (see Discussion).

We also asked which RGC types included I-RGCs. At E13, putative I-RGCs were highly enriched in 2 of 10 clusters, comprising 38-40\% of clusters 2 and $9,9-14 \%$ of clusters 3 and 5 , and $<2 \%$ of the other 6 clusters (Fig. 7d). In adults, RGCs expressing Tbr1, Mafb, Foxp2 and Neurod2 contained 3-4X more I-RGCs than RGCs expressing Eomes, Irx3 or Tfap2d. These results are consistent with previous observations that I-RGCs are morphologically and physiologically heterogenous but not uniformly distributed across types(Hong, Kim, \& Sanes, 2011; Johnson et al., 2021). Lastly, the WOT-predicted 625 relationship between E13 precursor RGC clusters and I-RGC-rich or -poor terminal 626 types were consistent with these patterns. The top six I-RGC-rich types (C4, C15, C19, 627 C20, C38, C45) derived $14 \%$ and $4 \%$ of their relative fate association from E13 clusters 6282 and 9, while the top six I-RGC-poor types (C8, C14, C18, C22, C31 and C41) derived 629 only 3.8 and $0.2 \%$ of their relative fate association from E13 clusters 2 and 9 . Thus, 
630 E13 clusters 2 and 9 are preferred precursors of adult types that are relatively rich in I631 RGCs.

632

633

634

Figure 7

a

E13

E14

E16
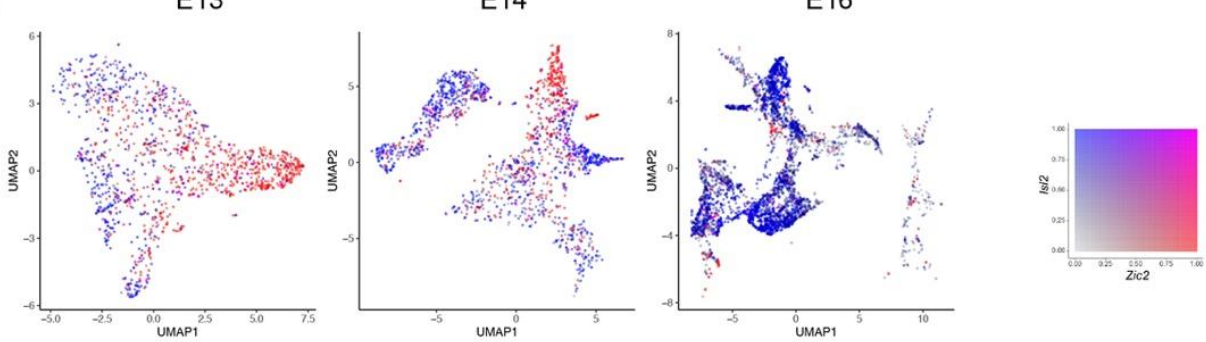

b

E13

E14

E16
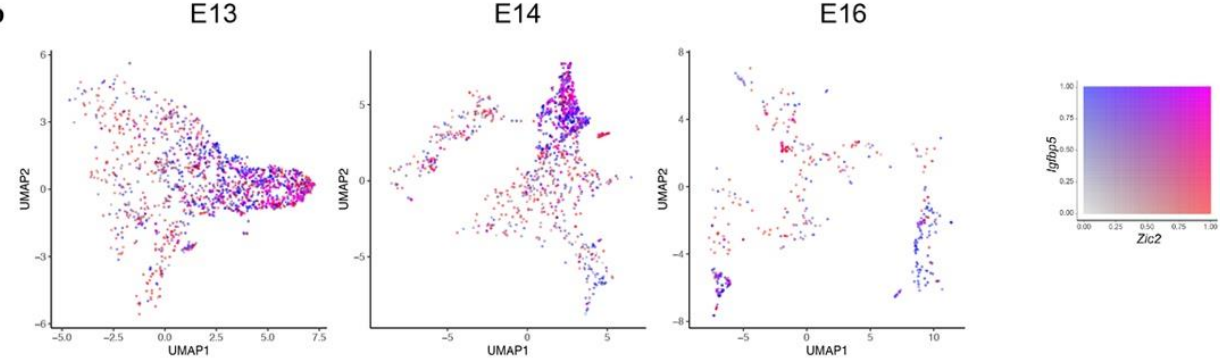

c

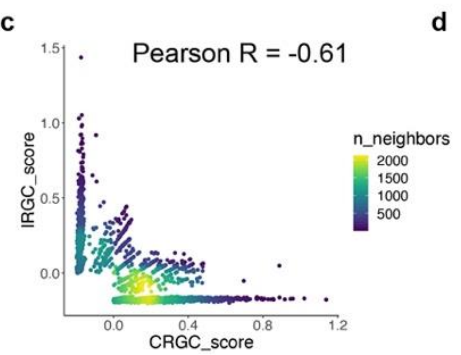

d

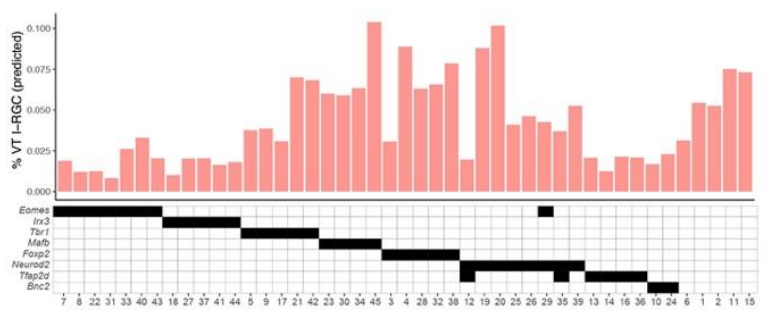

e

E13
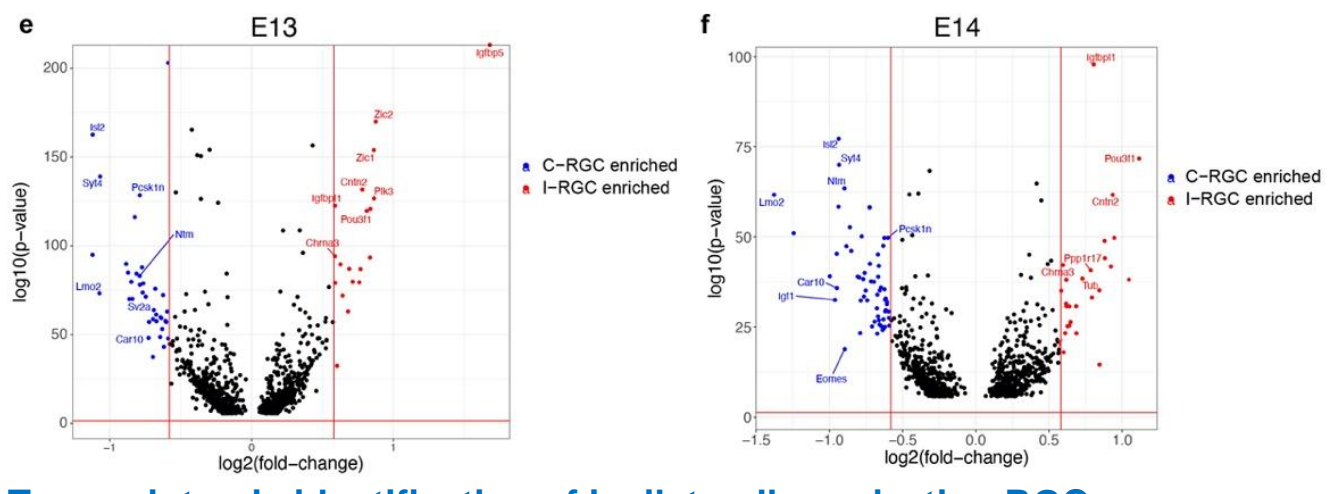

Figure 7. Transcriptomic identification of ipsilaterally projecting RGCs 
637 a. Zic2, an I-RGC marker and IsI2, a C-RGC marker, are expressed in a mutually exclusive 638 pattern at E13 (left), E14 (middle) and E16 (right). Zic2 is undetectable after E16 (Figure 7639 figure supplement 1a). Cells are colored based on a bivariate color scale representing co640 expression of two markers (colorbar, right).

641 b. Zic2 and Igfbp5, two I-RGC markers, are co-expressed at E13 (left) and E14 (middle). 642 Representation as in panel a.

643 c. Scatter-plot of gene signatures used to identify I-RGCs (y-axis) and C-RGCs (x-axis) at E13 644 are negatively correlated (Pearson $\mathrm{R}=-0.61$ ). Each dot corresponds to a cell, the color 645 represents the number of cells located at a particular $(x, y)$ location (see colorbar, right).

646 d. Barplot showing \% of putative I-RGCs (y-axis) within each of the 45 adult RGC types, 647 estimated by computing the descendants of E13 I-RGCs using WOT. RGC types are arranged 648 along the $x$-axis based on their membership of TF-groups shown in Fig. 6a (annotation matrix, 649 bottom).

650 e. Volcano plot showing DE genes (MAST test, $\mathrm{p}<10^{-6}$ ) between predicted I-RGCs and C651 RGCs at E13. The $x$ - and the $y$-axes show the fold-change and the $p$-value in log2- and log10units, respectively. Dots represent genes, with red and blue dots highlighting I- and C-RGC enriched genes respectively at fold-change $>1.5$ and Bonferroni corrected $p$-value $<5 \times 10^{-5}$. The two vertical bars correspond to a fold-change of 1.5 in either direction. Select I-RGC and CRGC enriched genes are labeled.

f. Same as panel e, for E14.

660 The staggering diversity of its neurons underlies the computational power of the nervous system. Accordingly, a major quest in developmental neurobiology is to 662 understand the mechanisms that diversify progenitors. A generally accepted way to deal 663 with this diversity is to divide neurons into classes, and then subdivide classes into 664 subclasses and subclasses into types (Zeng \& Sanes, 2017). While much has been 665 learned about how neural progenitors give rise to distinct neuronal classes, little is 666 known about how classes diversify into subclasses and types.

668 Here, we used mouse RGCs to address this issue. We recently generated a molecular 669 atlas that divided RGCs into 45 distinct types based on their patterns of gene 670 expression (Tran et al., 2019). We used this atlas here as a foundation to ask how these 671 types are specified during development. We conclude that the earliest precursor RGCs 672 are multipotential and exhibit continuous variation in transcriptomic identity, then 673 diversify into definitive types by a gradual process of fate restriction. Interestingly, these 674 features resemble those that have been discovered to control the generation of retinal 
675 cell classes from cycling progenitors (RPCs): multipotentiality, progressive restriction of 676 fate, and stochastic rather than deterministic fate choice (see Introduction). We 677 suggest that, at least in this case, similar strategies are used to generate cell classes 678 from mitotically active progenitors and cell types from postmitotic precursors.

Classes, subclasses and types

681 Definitions of neuronal class, subclass and type have been contentious (Yuste et al., 682 2020). In general, classes share general features of structure, function, molecular 683 architecture and location, whereas types comprise the smallest groups within classes 684 that can be qualitatively distinguished from other groups based on these and other 685 criteria. Subclasses lie in-between. For RGCs, class identity has been clear for a century, but inventories of subclasses and types have emerged only over the last few 687 decades, as high-throughput methods have been implemented for quantifying structural 688 (primarily dendritic morphology), functional (responses to an array of visual stimuli) and molecular properties (gene and transgene expression) of large numbers of RGCs. 690 Fortunately, to the extent that they have been compared, there is excellent concordance 691 among types defined by molecular, structural and physiological criteria (Bae et al., 2018; Goetz et al., 2021; Tran et al., 2019) (see www.rgctypes.org). Moreover, RGCs 693 of a single type exhibit a regular spacing, called a mosaic arrangement, in that they tend 694 to avoid other members of the same cell type whereas their association with members 695 of other types is random (Kay et al., 2012; Keeley et al., 2020; Rockhill et al., 2000). 696 The molecular basis of this property is poorly understood, but it provides an additional 697 criterion for defining a type. Thus, while no two RGCs are identical, and variation may 698 be continuous in some other structures (Cembrowski \& Spruston, 2019), there is strong 699 reason to believe that $R G C$ types are discrete.

\section{The adult RGC atlas}

702 Developmental trajectories of cell types cannot be better than the adult types at which 703 they are aimed. We have two reasons to believe that our adult RGC atlas (Tran et al., 704 2019) is accurate and complete. 
706 First, the atlas is based on detailed analysis of 35,699 cells, and is therefore powered to 707 detect types occurring at $\sim 0.1 \%$ frequency $(>40$ cells per type; 708 https://satijalab.org/howmanycells/). Results were stable over a variety of parameters 709 (Tran et al., 2019). Moreover, in the course of studies on responses of RGCs to injury, 710 we recently profiled an additional $\sim 120,000$ cells (A. Jacobi, N. Tran, W Yan and J.R.S, 711 in preparation), without identifying additional types.

713 Second, RGCs have now been classified by functional and structural properties, based 714 on physiological responses to visual stimuli (Baden et al., 2016; Goetz et al., 2021) and 715 serial section electron microscopy (Bae et al., 2018). The numbers of types defined in 716 these ways (47 in (Bae et al., 2018), 42 in (Goetz et al., 2021) and > 32 in (Baden et al., 717 2016)) match well to the 45-46 defined molecularly (Tran et al., 2019).

\section{Multipotentiality of precursor RGCs}

720 The multipotentiality of dividing progenitor cells can be demonstrated by indelibly 721 labeling a progenitor and then examining its progeny at a later stage. For mammals, 722 this was initially done by infecting single cells with a recombinant retrovirus encoding a 723 reporter gene that could be detected following multiple cell divisions (Price, Turner, \& 724 Cepko, 1987; Sanes, Rubenstein, \& Nicolas, 1986; Turner \& Cepko, 1987). More 725 recently, it has become possible to greatly increase throughput by tracking scars or 726 barcodes introduced by CRISPR/Cas9 (Baron \& van Oudenaarden, 2019; Espinosa727 Medina, Garcia-Marques, Cepko, \& Lee, 2019; McKenna et al., 2016). In sharp 728 contrast, conclusively demonstrating that a single postmitotic cell is multipotential would 729 require following a cell from an unspecified to a specified state, then turning back time, 730 watching it again, and asking if it acquired the same mature identity. Since this is 731 impossible, we used computational methods to draw tentative conclusions about the 732 extent to which newly postmitotic RGCs are committed to mature into a particular type 733 based on their transcriptomic profiles. Consequently, our results are based on inferred 734 rather than experimentally determined lineages. 
736 Our analysis proceeded in three steps. First, to ask whether RGCs were committed to a 737 particular fate before or shortly after they were born, we assessed transcriptomic 738 heterogeneity at a time when a large fraction was newly postmitotic (E13 and E14). We 739 found that heterogeneity was present but limited: 10 transcriptomic clusters were 740 distinguishable at E13 and 12 at E14. Thus, some heterogeneity is present in precursor 741 RGCs, but far less than would be required to specify type identity before or immediately 742 after their birth. A relevant issue is whether at these early stages the transcriptomic 743 variation among cells could reflect variation in their stages of differentiation, perhaps as

744 a consequence of different intervals between birthdate and sampling. Although we 745 cannot exclude this possibility, inter-cluster variability of key RGC-specific genes (e.g., 746 Rbpms) was no greater at early times than in adults (data not shown).

Second, we used a supervised classification approach to ask whether precursor RGC clusters mature into mutually exclusive sets of adult types. This model would imply an orderly, step-wise restriction of cell fates. However, our results indicate substantial overlap in the types derived from cells in different immature clusters. This result argues

752 against a deterministic model of diversification, and suggests that precursor RGCs are 753 incompletely committed to a specific type for a substantial period after they are 754 generated.

756 Third, we used optimal transport inference (WOT) to ask whether the multipotentiality 757 observed at the level of groups was also a property of individual cells. This approach 758 circumvents the limitation of the supervised classification approach, which compares 759 similarity only at the level of clusters. WOT utilizes time course scRNA-seq snapshots to 760 infer fate associations between individual cells sampled at different time points, without 761 reference to the clusters in which they reside (Schiebinger et al., 2019). While being 762 consistent with supervised classification results at the cluster level, WOT indicated that 763 the majority of individual RGCs were multipotential at E13 and E14. Of equal 764 importance, immature RGCs were not totipotential: the average predicted potential $(P)$ 765 was 11.6 at $E 13$, or $\sim 25 \%$ of the maximum possible value of 45 , and no RGCs had $P$ 
$766>30$. We conclude that single multipotential immature RGCs are biased in favor of 767 particular groups of adult RGC types.

\section{Progressive restriction of RGC fate}

770 Further analysis provided insight into the structure of multipotentiality among RGCs.

771 The adult RGC types associated with a precursor RGC were not a randomly chosen 772 subset; rather some were more likely to arise from a common precursor state ("fate 773 coupled") than others. This suggests a model in which RGC types arise via a 774 progressive decoupling of fates within multipotential precursors. Decoupling is 775 asynchronously, with different types emerging at different times. By modeling the 776 temporal kinetics of fate decoupling, we were able to estimate a tentative specification 777 time for each type - that is, the time at which precursors acquire a distinct 778 transcriptomic identity. Analysis of transcriptomic changes that occur during this 779 process, and the effects of visual experience on maturation, will be presented 780 elsewhere (K.S., I.E.W., S.B. and J.R.S., in preparation).

782 Our conclusions about fate restriction are based on analyzing cell states defined by 783 expression patterns of highly variable genes identified in the data (Figure $\mathbf{2 H}$ ). An 784 alternative and common view is that if a small set of genes is sufficient to define cell 785 state, they should be the focus of analysis. We believe this is an incorrect argument, 786 based on confusion between genes that determine a cell type or state, and those that 787 define it. The only way a small number of genes, whatever their functional role, can 788 exclusively define cell state is if they are expressed at very high levels. If this were the 789 case, they would be detected in our data and drive the clustering.

\section{Fate-restricted RGC subclasses}

792 For RGCs, class identity has been clear for a century, and type identity has been 793 solidified during over the last few decades, but criteria for defining subclasses remain 794 unclear. Tentative classifications have used molecular, physiological and morphological 795 criteria (Sanes \& Masland, 2015; Tran et al., 2019); In general, these criteria correlate 796 imperfectly with each other, the main exception being that ON and OFF RGCs 
797 (responding preferentially to increases and decreases in illumination, respectively) have 798 dendrites that arborize in the inner and outer portion of the inner plexiform layer 799 (Famiglietti \& Kolb, 1976).

801 The pattern of fate couplings between RGC types at E13-14 provides an alternative way 802 to define RGC subclasses - groups of RGC types that arise from the restriction of a 803 common transcriptionally defined precursor state. We identified transcription factors 804 selectively expressed within these subclasses. Our rationale was that among them 805 would be fate determinants, an idea that could be tested by conventional genetic 806 manipulations. Support for this idea is that there is already strong evidence that one 807 such factor is a fate determinant in mouse: Eomes is selectively expressed by ipRGCs 808 (and a few other types), and Eomes mutants fail to form ipRGCs although their retinas 809 are normal in many respects (C. A. Mao et al., 2014). This encourages the hope that 810 some of the other transcription factors in this set are also fate determinants. It will also 811 be interesting to determine whether members of fate-restricted subclasses share 812 structural or functional properties.

814 Laterality

815 The transcription factors $I s / 2$ and Zic2 are selective markers of embryonic RGCs that 816 project contralaterally or ipsilaterally, respectively, and are critical determinants of this 817 choice (Herrera et al., 2003; Pak et al., 2004). We found that their expression was 818 largely nonoverlapping in RGCs at E13, that they were co-expressed with previously 819 reported markers of contralaterally and ipsilaterally projecting RGCs, respectively. 820 Because few RGC axons reach the optic chiasm before E14, our results are consistent 821 with genetic evidence that this differential expression is a cause rather than a 822 consequence of the divergent choices the axons make at the chiasm. Among many 823 genes co-expressed with Is/2 or Zic2 may be others that play roles in this choice.

825 Zic2 is downregulated later in embryogenesis, so we selected some RGCs as putative I826 RGCs using genes known to be expressed in them (Wang et al., 2016), then used WOT 827 to infer the RGC types to which they gave rise. Our analysis suggests that I-RGCs 
828 comprise many differentiated types, consistent with previous results (Hong et al., 2011;

829 Johnson et al., 2021). Surprisingly, however, there were few if any genes differentially 830 expressed between the putative mature I- and C-RGCs. Assuming that WOT results 831 are valid - an assertion that can be tested directly in the future - this result suggests a 832 model in which newborn RGCs are doubly specified - by laterality and by type - but that 833 once axonal choice has been made the laterality program is shut down.

834

\section{Beyond the retina}

836 Generation of neuronal classes has been analyzed in many parts of the vertebrate 837 nervous system but we are aware of few reports on how classes diversify into types. A 838 recent study addressed this issue for primary sensory neurons and reached the 839 conclusion that newborn neurons in dorsal root ganglia are transcriptionally 840 unspecialized and become type-restricted as development proceeds (Sharma et al., 841 2020). Similarly, both excitatory neuronal subclasses appear to diversify postmitotically 842 in the mouse cerebral cortex (Di Bella et al., 2021; Lodato \& Arlotta, 2015), and there is 843 suggestive evidence that the same is true for interneuronal subclasses (Wamsley \& 844 Fishell, 2017). In all of these cases, it is attractive to speculate that diversification may 845 occur by a process of fate decoupling in subpopulations of distinct multipotential 846 precursors, akin to that documented here for RGCs. Our study provides a 847 computational framework for investigating this issue. 
Methods and Materials

\begin{tabular}{|c|c|c|c|c|}
\hline \multicolumn{5}{|c|}{ Key Resources Table } \\
\hline $\begin{array}{l}\text { Reagent } \\
\text { type } \\
\text { (species) } \\
\text { or } \\
\text { resource }\end{array}$ & $\begin{array}{l}\text { Designatio } \\
\mathbf{n}\end{array}$ & $\begin{array}{l}\text { Source or } \\
\text { reference }\end{array}$ & Identifiers & $\begin{array}{l}\text { Additiona } \\
\text { I } \\
\text { informati } \\
\text { on }\end{array}$ \\
\hline $\begin{array}{l}\text { strain, } \\
\text { strain } \\
\text { backgrou } \\
\text { nd (Mus } \\
\text { Musculus } \\
\text { ) }\end{array}$ & C57BL/6 & $\begin{array}{l}\text { Charles River or } \\
\text { Jackson Labs }\end{array}$ & $\begin{array}{l}\text { Cat\#JAX000664; RRID: } \\
\text { IMSR_JAX:000664 }\end{array}$ & \\
\hline Antibody & $\begin{array}{l}\text { Anti- } \\
\text { Thy1/anti- } \\
\text { CD90 } \\
\text { (rat } \\
\text { monoclon } \\
\text { al) }\end{array}$ & $\begin{array}{l}\text { ThermoFisher } \\
\text { Scientific }\end{array}$ & \#17-0902-82 & $1: 200$ \\
\hline Antibody & $\begin{array}{l}\text { Anti- } \\
\text { L1cam } \\
\text { (rat } \\
\text { monoclon } \\
\text { al) }\end{array}$ & $\begin{array}{l}\text { Miltenyi } \\
\text { Biotec }\end{array}$ & $\# 130-102-243$ & $1: 10$ \\
\hline Antibody & $\begin{array}{l}\text { Anti-CD90 } \\
\text { pre- } \\
\text { conjugate } \\
\text { d (Rat } \\
\text { monoclon } \\
\text { al) }\end{array}$ & Miltenyi Biotec & $\# 130-049-101$ & $1: 200$ \\
\hline
\end{tabular}




\begin{tabular}{|c|c|c|c|c|}
\hline Antibody & $\begin{array}{l}\text { Anti- } \\
\text { RBPMS } \\
\text { (guinea } \\
\text { pig } \\
\text { polyclonal } \\
\text { ) }\end{array}$ & $\begin{array}{l}\text { PhosphoSoluti } \\
\text { ons }\end{array}$ & \#1832-RBPMS & $1: 1000$ \\
\hline Antibody & $\begin{array}{l}\text { Anti-KI67 } \\
\text { (rabbit } \\
\text { monoclon } \\
\text { al) }\end{array}$ & $\begin{array}{l}\text { ThermoFisher } \\
\text { Scientific }\end{array}$ & \#MA5-14520 & $1: 250$ \\
\hline $\begin{array}{l}\text { chemical } \\
\text { compoun } \\
\text { d, drug }\end{array}$ & Fluoro-Gel & $\begin{array}{l}\text { Electron } \\
\text { Microscopy } \\
\text { Sciences }\end{array}$ & \#17985 & \\
\hline $\begin{array}{l}\text { Commerc } \\
\text { ial Assay } \\
\text { or Kit }\end{array}$ & $\begin{array}{l}\text { MACS Lar } \\
\text { ge Cell } \\
\text { Columns }\end{array}$ & Miltenyi Biotec & $\# 130-042-202$ & \\
\hline $\begin{array}{l}\text { Sequence } \\
\text { based } \\
\text { reagent }\end{array}$ & $\begin{array}{l}\text { Drop-seq } \\
\text { beads }\end{array}$ & $\begin{array}{l}\text { ChemGenes } \\
\text { Corporation }\end{array}$ & \#Macosko201110 & \\
\hline $\begin{array}{l}\text { Commerc } \\
\text { ial assay } \\
\text { or kit }\end{array}$ & $\begin{array}{l}\text { Papain } \\
\text { dissociatio } \\
\text { n system }\end{array}$ & Worthington & \#LK003160 & \\
\hline $\begin{array}{l}\text { Commerc } \\
\text { ial assay } \\
\text { or kit }\end{array}$ & $\begin{array}{l}\text { Chromium } \\
\text { Single Cell } \\
\text { 30Library } \\
\text { and Gel } \\
\text { Bead Kit v2, } \\
10 X \\
\text { Genomics } \\
\text { 16rxns }\end{array}$ & 10X Genomics & \# 120237 & \\
\hline $\begin{array}{l}\text { software, } \\
\text { algorithm }\end{array}$ & $\begin{array}{l}\text { Cell Ranger } \\
\text { v2.6.0 }\end{array}$ & 10X Genomics & $\begin{array}{l}\text { https://support.10xgenomics.com/ } \\
\text { single-cell-gene- } \\
\text { expression/software/downloads/lat } \\
\text { est }\end{array}$ & \\
\hline $\begin{array}{l}\text { software, } \\
\text { algorithm }\end{array}$ & $\begin{array}{l}\text { ImageJ (Fiji) } \\
\text { Version } \\
2.1 .0\end{array}$ & Fiji & https://imagej.net/Fiji & \\
\hline
\end{tabular}




\begin{tabular}{|l|l|l|l|l|}
\hline & & & & \\
\hline $\begin{array}{l}\text { Software, } \\
\text { algorithm }\end{array}$ & R 3.6 .2 & $\begin{array}{l}\text { The R } \\
\text { foundation }\end{array}$ & https://www.r-project.org/ & \\
\hline $\begin{array}{l}\text { Software, } \\
\text { algorithm }\end{array}$ & $\begin{array}{c}\text { RStudio } \\
1.3 .1056\end{array}$ & Rstudio & https://www.adobe.com & \\
\hline
\end{tabular}

Mice

871 All animal experiments were approved by the Institutional Animal Care and Use 872 Committees (IACUC) at Harvard University. Mice were maintained in pathogen-free 873 facilities under standard housing conditions with continuous access to food and water.

874 Animals used in this study include both males and females. A meta-analysis (not 875 shown) did not show any systematic sex-related effects in either differentially expressed 876 genes or cell-type proportions. For scRNA-seq and histology, we used C57BI/6J (JAX 877 \#000664). Embryonic and early post-natal C57BI/6J mice were acquired either from 878 Jackson Laboratories (JAX) from time-mated female mice or time-mated in-house. For 879 timed-matings, a male was placed with a female overnight and removed the following 880 morning (with the corresponding time recorded as day E0.5).

\section{Cell preparation}

884 RGCs were enriched from dissociated retinal cells as previously described with minor 885 modifications (Tran et al., 2019). All solutions were prepared using Ames' Medium with 886 L-glutamine and sodium bicarbonate (equilibriated with $95 \% \mathrm{O}_{2} / 5 \% \mathrm{CO}_{2}$ ), and all spin 887 steps were done at $450 \mathrm{~g}$ for 8 minutes. Retinas were dissected out in their entirety 888 immediately after enucleation and digested in $~ 80 \mathrm{U}$ of papain at $37 \mathrm{C}$, with the 889 exception of some E13 and E14 eyes which were digested whole, followed by manual 890 trituration in ovomucoid solution. Clumps were removed using a $40 \mu \mathrm{m}$ cell strainer and 
891 the cell suspension was spun down and re-suspended in Ames $+41 \%$ BSA at a 892 concentration of 10 million cells per $100 \mu$ l. Cells from E13, E14, E16, and P0 were 893 incubated for 15 minutes at room temperature with antibodies to Thy1 (also known as 894 CD90) and L1CAM pre-conjugated to the fluorophores APC (ThermoFisher Scientific \#17-0902-82) and PE (Miltenyi Biotec \#130-102-243), respectively. Cells were washed

896 with $6 \mathrm{ml}$ of Ames $+4 \%$ BSA, spun down and resuspended at a concentration of $\sim 7$ 897 million cells $/ \mathrm{ml}$, and calcein blue was added to label metabolically active cells.

Viable Thy1 or L1CAM positive cells were sorted using a MoFlo Astrios sorter into 900 $\sim 100 \mu \mathrm{l}$ of AMES $+4 \%$ BSA. Sorted cells were spun down a final time and resuspended in PBS $+0.1 \%$ BSA at a concentration of 500-2000 cells $\mu$ l. P5 RGCs were enriched using only CD90, with either magnetic-activated cell sorting (MACS) using large cell columns and CD90 pre-conjugated to microbeads (\#130-042-202 and \#130-049-101, 904 Miltenyi Biotec), fluorescence activated cell sorting (FACS) with CD90 pre-conjugated to 905 PE/Cy7 (ThermoFisher Scientific \#25-0902-81), or both.

906

907 Droplet-based single-cell RNA-seq

908

\section{Statement on replicates}

910 We profiled immature RGCs using scRNA-seq at five developmental time points: E13, 911 E14, E16, P0 and P5. At each age, data was collected from four replicate experiments. 912 Experiments at E13, E14, E16 and P0 involved two biological replicates (distinct mice). 913 Each of these biological replicates were further subdivided into two equal pools and the 914 cells were subjected to two different enrichment methods (anti-Thy1 and anti-L1cam). 915 Thus, each of these time points consisted of four replicate experiments. RGC 916 enrichment at P5 exclusively utilized anti-Thy1, but four biological replicate experiments 917 were performed. One of these was profiled using 10X and three of these were profiled 918 using Drop-seq. 
921 A subset of P5 RGC dataset was collected using Drop-seq (Macosko et al., 2015), 922 performed largely as described previously (Shekhar et al., 2016). Briefly, cells were 923 diluted to an estimated final droplet occupancy of 0.05 , and co-encapsulated in droplets 924 with barcoded beads, which were diluted to an estimated final droplet occupancy of 925 0.06. The beads were purchased from ChemGenes Corporation, Wilmington MA (\# 926 Macosko201110). Individual droplet aliquots of $2 \mathrm{ml}$ of aqueous volume (1 $\mathrm{ml}$ each of 927 cells and beads) were broken by perfluorooctanol, following which beads were 928 harvested, and hybridized RNA was reverse transcribed. Populations of 2,000 beads $929 \sim 100$ cells) were separately amplified for 14 cycles of PCR (primers, chemistry, and 930 cycle conditions identical to those previously described) and pairs of PCR products 931 were co-purified by the addition of 0.6x AMPure XP beads (Agencourt). Fifteen 932 experimental replicates were sequenced in total from 5 biological replicates using an 933 Illumina NextSeq 500. Read 1 was $20 \mathrm{bp}$; read 2 (paired-end) was $60 \mathrm{bp}$.

\section{$\underline{10 X \text { Genomics }}$}

936 Single cell libraries were prepared using the Single-cell gene expression 3' kit on the 937 Chromium platform (10X Genomics, Pleasanton, CA) following the manufacturer's 938 protocol. As our datasets were collected over a long period of time, we used a 939 combination of v1 (a single channel of P5 RGCs), v2 (E13, E14, E16, P0). Briefly, single 940 cells were partitioned into Gel beads in EMulsion (GEMs) in the 10X Chromium 941 instrument followed by cell lysis and barcoded reverse transcription of RNA, 942 amplification, enzymatic fragmentation, 5' adaptor attachment and sample indexing. On 943 average, approximately 8,000-12,000 single cells were loaded on each channel and 944 approximately 3,000-7,000 cells were recovered. Libraries were sequenced on the 945 Illumina HiSeq 2500 platforms at the Broad institute (Paired end reads: Read 1, 26 946 bases, Read 2, 98 bases).

Power analysis

949 An important question in all single-cell experiments is that of the number of cells to 950 profile. A widely used approach is the power analysis tool published by the Satija lab 951 (https://satijalab.org/howmanycells/). Fortunately, in this study we were also guided by 
952 our previous study of adult RGCs, where we had knowledge of the frequency 953 distribution of adult RGC types, with the rarest type being approximately $0.2 \%$ (Tran et 954 al., 2019). In that study, we also found that when classification is performed in a 955 supervised fashion based on an existing atlas, approximately 8000 RGCs were 956 sufficient to recover the accurate relative frequency distribution of $45 \mathrm{RGC}$ types. We 957 therefore aimed to profile $\sim 8000$ cell at each time point as our analysis involved 958 mapping immature RGCs to the adult atlas. With the exception of E13, all time points 959 contain 1.5-2X more cells than this target value.

\section{Histology}

963

\section{Tissue fixation}

965 Adult (P56) mice were intracardially perfused with $2-5 \mathrm{ml}$ of PBS followed by $15 \mathrm{ml}$ of $4 \%$ 966 PFA, followed by additional fixation of eyes for 15 minutes in 4\% PFA. P0 and P5 mice 967 were not perfused, rather eyes were fixed in 4\% PFA for 30 minutes. At E13, 14, and 16 968 embryos were fixed whole for 30 minutes in 4\% PFA, following which eyes were 969 removed. Following fixation eyes from all time points were transferred to PBS and 970 stored at $4 \mathrm{C}$ until subsequent use.

972 Sectioning

973 Cross sections for immunohistochemistry (IHC) were generated using a Leica CM1850 974 cryostat. For some early developmental time points eyes were kept whole for IHC, 975 otherwise retinas were either (1) dissected out in their entirety from eyes, or (2) the 976 cornea, iris and lens was removed leaving the sclera and retina intact. Tissues were 977 sunk in $30 \%$ sucrose overnight at $4 \mathrm{C}$, embedded in tissue freezing medium, and cryo978 sectioned into $25 \mathrm{~mm}$ slices. Slides with tissue sections were air dried for $\sim 3$ hours and 979 stored at $-80 \mathrm{C}$ until staining.

$\underline{\text { Immunohistochemistry (IHC) }}$ 
982 All IHC solutions were made up in PBS $+0.3 \%$ Triton-X, and all incubation steps were 983 carried out in a humidified chamber. Following a 1 hour protein block in 5\% Normal 984 Donkey Serum at room temperature, slides were incubated overnight at $4 \mathrm{C}$ with primary 985 antibodies, washed twice for 5 minutes each in PBS, incubated for 2 hours at room 986 temperature with secondary antibodies conjugated to various fluorophores (1:1000, 987 Jackson Immunological Research) and Hoechst (1:10000, Life Technologies), and 988 washed again twice for 5 minutes each in PBS before coverslipping with Fluoro-Gel 989 (\#17985, Electron Microscopy Sciences). Primary antibodies used include: guinea pig 990 anti-RBPMS (1:1000, \#1832-RBPMS, PhosphoSolutions), rabbit anti-KI67 (1:250, 991 \#MA5-14520, ThermoFisher Scientific), rat anti-L1CAM (1:10, \#130-102-243, Miltenyi 992 Biotec).

993

994 Imaging

995 All images were acquired using an Olympus Fluoview 1000 scanning laser confocal 996 microscope, with a 20X oil immersion objective and 2x optical zoom. Optical slices 997 were taken at $1 \mu \mathrm{m}$ steps. Fiji was used to pseudocolor each channel and generate a 998 maximum projection from image stacks. Brightness and contrast was adjusted in Adobe 999 Photoshop.

1000

1001

\section{Alignment and quantification of gene expression in single cells}

1002

1003

All single-cell libraries were aligned to the UCSC mm10 transcriptomic reference $(M$. 1004 musculus) and gene expression matrices were quantified using standard protocols 1005 described previously. For the single-cell libraries generated using the 10X platform 1006 (E13, E14, E16, P0 and P5), these steps were performed using cellranger v2.1.0 (10X 1007 Genomics). For the single-cell libraries generated using Drop-seq (P5), we used Drop1008 1009 1010 1011 seq tools (v1.12; (Macosko et al., 2015)), following procedures described earlier (Shekhar et al., 2016). Alignment and quantification was done for each sample library separately to generate a genes $X$ cells expression matrix of transcript counts. These matrices were column-concatenated for further analysis. 
1013 We retained cells that expressed at least 700 genes, resulting in 98,452 cells. We also 1014 removed genes expressed in fewer than 10 cells. The resulting $M$ genes $X N$ cells 1015 matrix of UMI counts $C_{m n}$ was normalized along each column (cell) to sum to 8,340, the 1016 median of the column sums resulting in a normalized matrix $X_{m n}$. This was followed by 1017 the transformation $X_{m n} \leftarrow \log \left(X_{m n}+1\right)$.

1018

\section{Overview of clustering analysis}

1020

102

The following procedure was adopted to perform batch correction, dimensionality 1022 reduction and clustering throughout the paper. The procedure was first applied on the 1023 entire dataset to separate RGCs from other cell classes, and then to RGCs at each age 1024 to identify transcriptomically distinct groups.

1025

1026

1027

1028

1029

1030

$$
\begin{gathered}
\mu_{m}=\frac{1}{N} \sum_{n} C_{m n} \\
\sigma_{m}^{2}=\frac{1}{N} \sum_{n}\left(C_{m n}-\mu_{m}\right)^{2} \\
C V_{m}=\frac{\sigma_{m}}{\mu_{m}}
\end{gathered}
$$

1031

1032 For a given $\mu_{m}$, the Gamma-Poisson model predicts a "null" coefficient of variation $1033\left(C V_{m}^{\text {null }}\right)$ arising from a combination of Poisson "shot" noise and large variations in library 1034 size, assumed to be due to technical reasons,

1035

$$
C V_{m}^{\text {null }}=\frac{1}{\mu_{m}}+\frac{1}{\alpha}
$$


Here, $\alpha$ is the shape parameter of a Gamma-distribution fit to the distribution of normalized library sizes $T_{n}$ (using the R package MASS),

$$
T_{n}=\frac{\sum_{m} C_{m n}}{\sum_{m, n} C_{m n}}
$$

1040

1041 In practice, $C V_{m}^{\text {null }}$ serves as a tight lower-bound for empirically observed values of $1042 C V_{m}$ across the full range of $\mu_{m}$. This enables us to compute for each gene $m$, a

1043 deviation score $d_{m}=\log \frac{C V_{m}}{C V_{m}^{\text {null }}}$, quantifying the extent to which its observed coefficient 1044 of variation exceeds the predicted null model. Highly-variable genes (HVGs) are 1045 selected if they satisfy $d_{m}>\operatorname{Mean}\left(d_{m}\right)+0.8 \operatorname{Std}\left(d_{m}\right)$.

2. Batch correction and Dimensionality Reduction: We subsetted the rows of the 1048 expression matrix $X_{m n}$ to the HVGs identified in Step 1. As our data comprised cells sampled at different developmental ages as well as multiple biological replicates within each age, we used Liger, a non-negative matrix factorization technique, to embed the data in a reduced dimensional latent space of shared factors (Welch et al., 2019). Liger computes a factorized representation for each matrix that separate "shared" and "dataset-specific" gene expression modules (factors). We use Liger's normalized $H$ factor loadings for cells to build a nearest neighbor graph and define clusters.

As in any matrix factorization technique, Liger requires the user to choose $k$, the dimensionality of the latent space. To find $k$, we use a Random Matrix Theory approach (outlined in (Peng et al., 2017)). Briefly, $k$ is estimated as the number of eigenvalues of the sample gene-gene correlation matrix that exceed the $99^{\text {th }}$ percentile of the distribution of the largest eigenvalue of a random Hermitian matrix of the same dimensions. This is given by the Tracy Widom distribution. For these calculations, we used the R package RMTstat. 
3. Clustering and 2D Visualization: To cluster cells based on transcriptomic 1066 similarity, we first built a nearest-neighbor graph on the cells based on their normalized $H$ factor coordinates computed using Liger. The number of nearest neighbors was chosen to be 30 . The edges were weighted based on the Jaccard overlap metric and graph clustering was performed using the Louvain algorithm, as described previously (Shekhar et al., 2016). The normalized $H$ factor coordinates were also used as input to project cells on to a nonlinear 2D space using the Uniform Manifold Approximation and Projection algorithm (UMAP; (Becht et al., 2019)). Graph-construction, clustering and the UMAP projection were performed using the R packages FNN, igraph and umap, respectively.

We began by clustering the full dataset combining all ages using the procedure outlined above. We identified groups of clusters corresponding to cell classes, which included retinal ganglion cells (RGCs; Rbpms, Slc17a6, Sncg, Nefl), microglia (P2ry12, C1qa-C, Tmem119), photoreceptors (Otx2, Gngt2, Gnb3\}), amacrine cells (Tfap2a, Tfap2b, Onecut2), anterior segment cells (Mgp, Col3a1,lgfbp7), cycling progenitors (Ccnd1, Fgf15, Hes5) and neurogenic progenitors (Hes6, Ascl1, Neurog2). Deeper annotation (e.g. of RGC type) was not done at this stage. No other cellular classes were identified. 3 clusters comprising fewer than $1.2 \%$ of the cells expressed markers of more than one class. These were flagged as doublets and removed from further analyses.

\section{Defining RGC precursor heterogeneity at each age}

RGC precursors at each age were separately analyzed following the clustering pipeline outlined previously. When implementing Liger, each biological replicate was regarded as a separate batch. The nominal clusters identified by the Louvain algorithm were refined as follows:

1. Removing contaminants: Clusters were flagged for further examination if they did not exclusively express RGC-specific markers (e.g. Rbpms, Slc17a6, Sncg, 
1095

1096

1097

1098

1099

1100

1101

1102

1103

1104

1105

1106

Neff). These clusters were small (typically $<1-2 \%$ of cells) and in all cases expressed non-RGC markers (e.g. P2ry12 or Tfap2b). These cells, which likely reflect trace contaminants, were discarded from further analysis.

2. Merging proximal clusters: Transcriptomic relationships between nominated clusters were visualized on a dendrogram computed using hierarchical clustering, as noted above above. Neighboring clusters on the dendrogram, which were leaves in a terminal branch, were assessed for differential expression using the MAST differential expression (DE) test (Finak et al., 2015). A gene $g$ was regarded as significantly DE between clusters $\mathcal{C}_{a}$ and $\mathcal{C}_{b}$ if it satisfied $\left|\log F C_{g}\left(\mathcal{C}_{a}, \mathcal{C}_{b}\right)\right|>0.5$ and MAST $p$-value was less than $10^{-5}$ (FDR corrected), where:

$$
\log F C_{g}\left(\mathcal{C}_{a}, \mathcal{C}_{b}\right)=\ln \left(\frac{\left|\mathcal{C}_{b}\right| \sum_{n \in \mathcal{C}_{a}} X_{g n}}{\left|\mathcal{C}_{a}\right| \sum_{n \in \mathcal{C}_{b}} X_{g n}}\right)
$$

1108 is defined to be the log-fold change in expression. Clusters that showed fewer than 10 1109 significant DE genes were merged. In this manner, we identified 10 RGC clusters at $1110 \mathrm{E} 13,12$ at E14, 19 at E16, 27 at P0, and 38 at P5. Using MAST, we identified DE genes 1111 that distinguished each cluster against the rest at any given age.

\section{Quantifying RGC diversity at different ages}

We quantified the molecular diversity of RGCs based on clusters at each stage using 3 measures of population diversity - The Rao index (Fig. 2), the Shannon index, and the

1117 Simpson index (Figure 2-figure supplement 1). For $N$ clusters with relative 1118 frequencies $p_{1}, p_{2}, \ldots, p_{N}$, these indices are defined as follows,

1. Let $d_{i j}$ be a distance measure between clusters $i$ and $j\left(0 \leq d_{i j} \leq 1\right)$. The Rao index is defined as, 


$$
R=\sum_{i \neq j} d_{i j} p_{i} p_{j}
$$

1123 We used varying number of genes $(\approx 1200-3000)$ to calculate $d_{i j}$. The computed Rao 1124 index was insensitive to these variations.

2. The Shannon index is defined as,

$$
H=-\sum_{i} p_{i} \log p_{i}
$$

3. The Simpson index is defined as,

$$
S=\sum_{i} p_{i}^{2}
$$

1131 While the Rao and Shannon indices increase with diversity, the Simpson index 1132 decreases with diversity.

\section{Analysis of cluster distinctiveness}

1135 We quantified the mutual separation of clusters at each age using two approaches:

1. Multi-class classification: We trained a multi-class classifier ( $R$ package xgboost) at each age on $50 \%$ of the cells using their cluster IDs. The remaining $50 \%$ of the cells were used to test the learned classifier and estimate a classification error per cluster, which were averaged at each age. As clusters become better separated, the average classification error decreases.

2. Relative positions in PC space: At each age, the top 20 PCA coordinates were first standardized by z-scoring. For each cluster $C$ at a given age, we computed two quantities:

a. $r_{C}$, the median of euclidean distances of each cell from the cluster centroid in the standardized PCA coordinates. 
b. $d_{C}$, the median of euclidean distances of each cell from the centroid of the nearest external cluster.

For a cluster $\mathrm{C}$, a low of value $r_{C} / d_{C}$ indicates a higher degree of separatedness. Averaging this metric across all the clusters at a given age quantifies the degree to which clusters are separated in the UMAP representation.

\section{Relating clusters across ages using supervised classification}

\section{Analysis Overview}

To distinguish between "specified" and "non-specified" modes of diversification (Figure 3), we first used a supervised classification approach to associate immature RGC clusters at young ages (tests) to cluster IDs determined at older ages (references). We used XGBoost, a decision-tree based ensemble learning algorithm (T. Chen \& Guestrin, 2016), to train multi-class classifiers on reference clusters, and used these to assign labels to individual test RGCs.

Two kinds of references were used: (1) Classifiers trained on the adult (P56) clusters were used to assign immature RGCs at each of the five developmental ages (five separate analyses) to adult labels. (2) Classifiers trained on E14, E16, P0, and P5 clusters were used to assign E13, E14, E16, and P0 RGCs to labels corresponding to the previous age, respectively (four separate analyses). The correspondence between classifier assigned labels and cluster IDs of test RGCs were visualized using confusion matrices (e.g. Figure 3d-h) and quantified using two metrics - the Adjusted Rand Index (ARI) and Normalized Conditional Entropy (NCE) metrics, described below.

\section{Classification Overview}

To describe our classification analysis, we introduce some notation to facilitate a description in general terms. Let $\mathcal{A}^{R}$ and $\mathcal{A}^{T}$ denote the reference and the test atlases for the purpose of supervised classification. The number of cells (i.e. RGCs) contained in the reference and test atlases are denoted $\left|\mathcal{A}^{R}\right|$ and $\left|\mathcal{A}^{T}\right|$, respectively. $\mathcal{A}^{R}$ and $\mathcal{A}^{R}$ could correspond to any pair of ages as described above. Without loss of generality, let 
1178 us assume that $\mathcal{A}^{R}$ contains $r$ transcriptomic clusters denoted $\left\{\mathcal{C}_{1}^{R}, \mathcal{C}_{2}^{R}, \ldots \mathcal{C}_{r}^{R}\right\}$.

1179 Similarly, $\mathcal{A}^{R}$ is assumed to contain $t$ transcriptomically defined clusters denoted $\left\{\mathcal{C}_{1}^{T}\right.$, $\left.1180 \mathcal{C}_{2}^{T}, \ldots \mathcal{C}_{t}^{T}\right\}$.

1183 Each cell in our dataset is the member of a particular atlas, and is assigned to a single 1184 cluster within the atlas based on its transcriptome. The transcriptome of each cell is a 1185 vector (denoted using lowercase boldface symbols, e.g. $\boldsymbol{u}$ or $\boldsymbol{v}$ ) with number of 1186 elements equal to the number of HVGs (the features used for classification). Let 1187 cluster $(\boldsymbol{u})$ denote the transcriptionally assigned of cell $\boldsymbol{u}$. For example, the following 1188 statement, 1189

$$
\boldsymbol{u} \in \mathcal{A}^{T}, \quad \text { cluster }(\boldsymbol{u})=\mathcal{C}_{k}^{T}
$$

1191 translates to "Cell $\boldsymbol{u}$ in atlas $\mathcal{A}^{T}$ is a member of cluster $\mathcal{C}_{k}^{T}$." Our goal is to assign each 1192 cell $\boldsymbol{u} \in \mathcal{A}^{T}$ a second ID cluster' $^{\prime}(\boldsymbol{u})$ based on its transcriptomic correspondence to the 1193 reference atlas $\mathcal{A}^{R}$. We perform this via an XGBoost classifier trained on $\mathcal{A}^{R}$ and 1194 applied it to every cell in $\mathcal{A}^{T}$, allowing us to infer transcriptomic correspondences 1195 between the two sets of clusters. The main steps are as follows,

- The expression matrices in $\mathcal{A}^{R}$ and $\mathcal{A}^{T}$ are z-scored along each feature. The initial set of features are chosen as the common HVGs in the two atlases. Parameters are adjusted to select the common top 2000-3000 HVGs. 


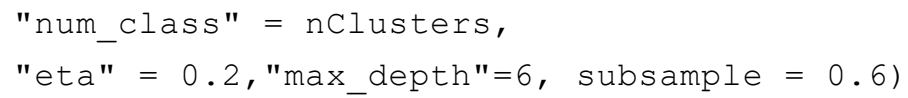

- When applied to a cell vector $\boldsymbol{u}$, the classifier $\operatorname{Class}_{0}^{R}$ (or $\operatorname{Class}_{0}^{T}$ ) returns a vector of $\boldsymbol{p}=\left(p_{1}, p_{2}, \ldots\right)$ of length $r$ (or $\left.t\right)$ with entries representing probability values of predicted cluster memberships in the corresponding atlas. We use these values to compute the "softmax" assignment of $\boldsymbol{u}$, so that $\operatorname{cluster}^{\prime}(\boldsymbol{u})=\operatorname{argmax}_{i} p_{i}$.

- Post training, $\operatorname{Class}_{0}^{R}$ and $\operatorname{Class}_{0}^{T}$ are evaluated on the respective validation sets. Using the predicted cluster assignments of the "held out" cells, we compute for each cluster in $\mathcal{A}^{R}$ and $\mathcal{A}^{T}$ the error-rate, defined as the fraction of held-out cells that were misclassified. If the error-rate for any cluster was higher than $10 \%$, the classifier is retrained by artificially upsampling cells from the high error-rate clusters. In the final classifiers, the cluster-specific error rates were typically 1 $4 \%$, and in no case exceeded $10 \%$.

- The top 500 discriminatory features (genes) are identified based on average information gain (using the function xgb. importance) for each of $\operatorname{Class}_{0}^{R}$ and $\operatorname{Class}_{0}^{T}$. These gene sets are denoted as $\mathcal{G}^{R}$ and $\mathcal{G}^{T}$ respectively.

- The common features $\mathcal{G}=\mathcal{G}^{R} \cap \mathcal{G}^{T}$ are used to train a third classifier $\operatorname{Class}^{R}$ on the reference atlas $\mathcal{A}^{R}$. This ensures that inferred transcriptomic correspondences are based on "core" gene expression programs that are conserved at both time points rather than temporally regulated genes.

- Finally, $\operatorname{Class}^{R}$ is applied to each cell $\boldsymbol{u} \in \mathcal{A}^{T}$ to generate predicted labels $\operatorname{cluster}^{\prime}(\boldsymbol{u})$. Global transcriptional correspondence were visualized using confusion matrices between cluster IDs $\operatorname{cluster}(\boldsymbol{u}) \in\left\{\mathcal{C}_{1}^{T}, \mathcal{C}_{2}^{T}, \ldots \mathcal{C}_{t}^{T}\right\}$ and reference assignments $\operatorname{cluster}^{\prime}(\boldsymbol{u}) \in\left\{\mathcal{C}_{1}^{R}, \mathcal{C}_{2}^{R}, \ldots \mathcal{C}_{r}^{R}\right\}$, and their correspondence was quantified using metrics described below.

\section{Quantifying cluster correspondence using global and local metrics}

Let $N_{i j}$ denote the number of cells in $\mathcal{A}^{T}$ that are part of transcriptomic cluster $\mathcal{C}_{j}^{T}$, and are assigned by $\operatorname{Class}^{R}$ to reference cluster $\mathcal{C}_{i}^{R}$. Thus, 


$$
N_{i j}=\#\left\{\operatorname{cluster}^{\prime}(\boldsymbol{u})=C_{i}^{R}, \operatorname{cluster}(\boldsymbol{u})=C_{j}^{T} \forall \boldsymbol{u} \in \mathcal{A}^{T}\right\}
$$

1240

$1241 N_{i j}$ defines a contingency table, whose marginal sums are defined as,

1242

$$
\begin{aligned}
& a_{i}=\sum_{j=1}^{t} N_{i j} \\
& b_{j}=\sum_{i=1}^{r} N_{i j}
\end{aligned}
$$

1243

1244 Let $N=\sum_{i, j} N_{i j}=\left|\mathcal{A}^{T}\right|$, the number of cells in $\mathcal{A}^{T}$. Then the Adjusted Rand index (ARI) 1245 corresponding to the assignments can be evaluated using the following equation, 1246

$$
A R I=\frac{\sum_{i j}\left(\begin{array}{c}
N_{i j} \\
2
\end{array}\right)-\left[\sum_{i}\left(\begin{array}{c}
a_{i} \\
2
\end{array}\right) \sum_{j}\left(\begin{array}{c}
b_{j} \\
2
\end{array}\right)\right] /\left(\begin{array}{c}
N \\
2
\end{array}\right)}{\frac{1}{2}\left[\sum_{i}\left(\begin{array}{c}
a_{i} \\
2
\end{array}\right)+\sum_{j}\left(\begin{array}{c}
b_{j} \\
2
\end{array}\right)\right]-\left[\sum_{i}\left(\begin{array}{c}
a_{i} \\
2
\end{array}\right) \sum_{j}\left(\begin{array}{c}
b_{j} \\
2
\end{array}\right)\right] /\left(\begin{array}{c}
N \\
2
\end{array}\right)}
$$

1247 The $A R I$ ranges from 0 and 1, with extremes corresponding to random association and $12481: 1$ correspondences between $\mathcal{A}^{R}$ and $\mathcal{A}^{T}$, respectively. The $\mathrm{ARI}$ can technically also 1249 take on negative values for certain scenarios, but these are not observed in our data.

1251 As an alternative, we also used the Normalized Conditional Entropy (NCE), an 1252 information-theoretic measure. The NCE quantifies the extent to which knowledge of the 1253 value of cluster $^{\prime}(\boldsymbol{u})$ reduces the uncertainty (measured in information bits) about the 1254 value of $\operatorname{cluster}(\boldsymbol{u})$ for $\boldsymbol{u} \in \mathcal{A}^{T}$.

1255

1256 We introduce probability weights $q_{i j}$ and the corresponding marginals $q_{i, .}$ and $q_{., j}$ as 1257 follows,

1258

$$
q_{i j}=\frac{N_{i j}}{N}
$$




$$
\begin{aligned}
q_{i, .} & =\frac{a_{i}}{N} \\
q_{., j} & =\frac{b_{j}}{N}
\end{aligned}
$$

1260 The Conditional Entropy (CE) is then given by the expression (Cover and Thomas, 1261 1991),

1262

$$
H\left(\operatorname{cluster}(\boldsymbol{u}) \mid \operatorname{cluster}^{\prime}(\boldsymbol{u})\right)=-\sum_{i j} q_{i j} \log \frac{q_{i j}}{q_{i, .}}
$$

1263

1264 Note that $\mathrm{CE}$ is asymmetric, i.e. $H\left(\operatorname{cluster}(\boldsymbol{u}) \mid \operatorname{cluster}^{\prime}(\boldsymbol{u})\right) \neq$ $1265 H\left(\operatorname{cluster}^{\prime}(\boldsymbol{u}) \mid \operatorname{cluster}(\boldsymbol{u})\right)$. One notes that $H=0$ if for each cluster $i \in\{1, \ldots, r\}, q_{i j}=$ $1266 \delta_{i, k_{i}}$ for a single cluster $k_{i}$ where $\delta_{l m}$ is the Kronecker delta defined as, 1267

$$
\begin{aligned}
& \delta_{i j}=1, \text { if } i=j \\
& \delta_{i j}=0, \text { if } i \neq j
\end{aligned}
$$

1268

1269 Finally, NCE is defined as,

1270

$$
N C E=\frac{H\left(\text { cluster }(\boldsymbol{u}) \mid \operatorname{cluster}^{\prime}(\boldsymbol{u})\right)}{H(\operatorname{cluster}(\boldsymbol{u}))}
$$

1271

1272 where $H($ cluster $(\boldsymbol{u}))=-\sum_{j} q_{., j} \log q_{., j}$ is the Shannon entropy. Due to this 1273 normalization, NCE values range from 0 to 1, with extremes corresponding to fully 1274 specific mapping or random association, respectively between $\mathcal{A}^{R}$ and $\mathcal{A}^{T}$. $A R I$ and 1275 NCE are inversely related. Unlike $A R I$, however, NCE is able to detect specificity in 1276 both many:1 and 1:1 mappings. ARI returns a value lower than 1 for specific mappings 1277 if the number of clusters in $\mathcal{A}^{R}$ and $\mathcal{A}^{T}$ are not equal. 
$1279 A R I$ and NCE quantify global correspondences between $\mathcal{A}^{R}$ and $\mathcal{A}^{T}$. We also computed 1280 a local metric, the Occupancy Fraction (OF) that quantified whether individual reference 1281 labels $\mathcal{C}_{i}^{R}$ were distributed in a "localized" or "diffuse" manner between test clusters $1282 \in\left\{\mathcal{C}_{1}^{T}, \mathcal{C}_{2}^{T}, \ldots \mathcal{C}_{t}^{T}\right\}$.

1283

$$
O F\left(\mathcal{C}_{i}^{R}\right)=\frac{1}{t}\left[\frac{1}{\sum_{j}\left(\frac{q_{i j}}{q_{i, .}}\right)^{2}}\right]
$$

1284

1285 Note that the term $\frac{q_{i j}}{q_{i_{i .}}}$ is simply the fraction of the total test cells belonging to test cluster $1286 \mathcal{C}_{j}^{T}$ that are assigned to reference cluster $\mathcal{C}_{i}^{R}$ by the classifier. Defined this way, the term 1287 in the square brackets computes an occupation number that ranges from 1 to $t$ and can 1288 be interpreted as the number of test clusters that are specifically associated with $\mathcal{C}_{i}^{R}$. 1289 Division by $t$ the number of test clusters, therefore converts this number into a fraction. 1290 129 1292 1293 1294 1295 1296 1297 1298 1299 1300 1301 1302 1303 1304 Overview of Waddington Optimal Transport (Waddington-OT) analysis To identify fate relationships among maturing RGCs used Waddington-OT (Schiebinger et al., 2019) a recently developed framework that is rooted in Optimal Transport theory (Villani, 2009). Waddington-OT does not rely on clustering, and therefore is able to identify ancestor-descendant relationships between any pair of temporally separated RGCs in our data.

At its heart, Waddington-OT models cellular transcriptomes $\boldsymbol{u}$ measured at a given age $t$ as a probability distribution in gene expression space $\mathbb{P}_{t}(\boldsymbol{u})$ (note that $\boldsymbol{u}$ may represent the original gene expression space or a reduced dimensional embedding estimated via Principal Component Analysis or Diffusion Maps.). This probability distribution evolves with time, as cells differentiate and mature. Different temporal measurements collected at times $\ldots, t_{i-1}, t_{i}, t_{i+1}, \ldots$ represent temporal snapshots of the corresponding cell distributions $\ldots, \mathbb{P}_{t_{i-1}}, \mathbb{P}_{t_{i}}, \mathbb{P}_{t_{i+1}}, \ldots$. Unfortunately, as each cell can only be measured once, the measurement at different times are from different cells. Therefore, for a 
1306 particular cell $\boldsymbol{u}$ at time $t_{i}$, it is not clear which cell(s) at time $t_{i-1}$ is likely to be its 1307 ancestor(s) and which cell(s) at time $t_{i+1}$ are likely to be descendant(s). It is this 1308 problem at Waddington-OT addresses.

1309

1310 Briefly, for a given pair of consecutive transcriptomic snapshopts $\mathbb{P}_{t_{i}}(\boldsymbol{u})$ and $\mathbb{P}_{t_{i+1}}(\boldsymbol{v})$, 1311 we wish to estimate the joint distribution $\Pi_{t_{i}, t_{i+1}}(\boldsymbol{u}, \boldsymbol{v})$, representing the probability that a 1312 cell having an expression vector $\boldsymbol{u}$ at time $t_{i}$ transitions to a cell with an expression 1313 vector $\boldsymbol{v}$ at time $t_{i+1} \cdot \Pi_{t_{i}, t_{i+1}}(\boldsymbol{u}, \boldsymbol{v})$ is also called the temporal coupling, which, owing to 1314 the destructive nature of scRNA-seq assays, is not directly observable. Under the 1315 assumption that cells move short distances in transcriptomic space when $\Delta t_{i}=t_{i+1}-t_{i}$ 1316 is "reasonably close", Waddington-OT estimates $\Pi_{t_{i}, t_{i+1}}(\boldsymbol{u}, \boldsymbol{v})$, as the solution to the 1317 following convex optimization problem,

1318

$$
\begin{array}{r}
\widehat{\boldsymbol{\Pi}}_{t_{i}, t_{i+1}}=\operatorname{argmin}_{\Pi} \sum_{\boldsymbol{u} \in \mathcal{A}^{t}} \sum_{\boldsymbol{v} \in \mathcal{A}^{t}} c(\boldsymbol{u}, \boldsymbol{v}) \boldsymbol{\Pi}(\boldsymbol{u}, \boldsymbol{v})-\epsilon \iint \boldsymbol{\Pi}(\boldsymbol{u}, \boldsymbol{v}) \log \boldsymbol{\Pi}(\boldsymbol{u}, \boldsymbol{v}) d \boldsymbol{u} d \boldsymbol{v} \\
+\lambda_{1} K L\left[\sum_{\boldsymbol{u} \in \mathcal{A}^{t}} \boldsymbol{\Pi}(\boldsymbol{u}, \boldsymbol{v}) \| d \widehat{\mathbb{P}}_{t_{i+1}}(\boldsymbol{v})\right]+\lambda_{1} K L\left[\sum_{\boldsymbol{v} \in \mathcal{A}^{t_{i+1}}} \boldsymbol{\Pi}(\boldsymbol{u}, \boldsymbol{v}) \| d \widehat{\mathbb{Q}}_{t_{i}}(\boldsymbol{u})\right]
\end{array}
$$

1319

1320 In the above equation,

- $\widehat{\mathbb{P}}_{t_{i+1}}(\boldsymbol{v})$ is an empirical distribution constructed from $\mathcal{A}^{t_{i+1}}$, which denotes the 1323

$$
\widehat{\mathbb{P}}_{t_{i+1}}(\boldsymbol{v})=\frac{1}{\mid \mathcal{A}^{t_{i+1} \mid}} \sum_{x_{i} \in \mathcal{A}^{t_{i+1}}} \delta\left(\boldsymbol{v}-\boldsymbol{x}_{\boldsymbol{i}}\right)
$$

1324 where $\delta(\boldsymbol{v}-\boldsymbol{x})$ denotes the Dirac delta function, a probability distribution placing all its mass at the location $x$.

- $\widehat{\mathbb{Q}}_{t_{i}}(\boldsymbol{u})$ is the cell distribution at $t_{i}$ rescaled by the relative growth rate to account for cell division/death, 


$$
\widehat{\mathbb{Q}}_{t_{i}}(\boldsymbol{u})=\widehat{\mathbb{P}}_{t_{i}}(\boldsymbol{u}) \frac{g(\boldsymbol{u})^{t_{i+1}-t_{i}}}{\int g(\boldsymbol{u})^{t_{i+1}-t_{i}} d \widehat{\mathbb{P}}_{t_{i}}}
$$

1329

1330

1331

1332

1333

1334

1335

1336

1337

1338

1339

1340

1341

1342

1343

1344

1345

1346

1347

1348

1349

1350

1351

1352

1353

1354

1355

1356

- Here $g(\boldsymbol{u})$ represents the relative growth rate of cell $\boldsymbol{u}$ in the time interval $\left(t_{i}, t_{i+1}\right)$ and is estimated within the framework of unbalanced optimal transport (Chizat, Peyré, Schmitzer, \& Vialard, 2018). For more details, we refer the reader to the supplementary information of (Schiebinger et al., 2019).

- $c(\boldsymbol{u}, \boldsymbol{v})$ is a cost function defined as the euclidean distance $|\boldsymbol{u}-\boldsymbol{v}|^{2}$. The first term of the objective function minimizes the cost function weighted by the temporal couplings, which may be interpreted as the transport distance between the distributions $\widehat{\mathbb{P}}_{t_{i}}$ and $\widehat{\mathbb{P}}_{t_{i+1}}$ (also known as the Wasserstein distance).

- The second term on the RHS represents entropic regularization, and $\epsilon$ is the corresponding strength. Classic OT identifies "deterministic" couplings in that one cell at $t_{i}$ is transported to a single cell at $t_{i+1}$. Introduction of the entropic regularization term makes this problem non-deterministic, capturing the notion that there may exist immature cells whose fate is not completely determined. Our inferences of multipotentiality is directly a consequence of adding this entropic regularization term. Additionally, entropic regularization also makes the problem strongly convex, which is computationally beneficial.

- The third and the fourth terms are features of unbalanced optimal transport, where equality constraints on the marginals (a consequence of mass conservation) are relaxed. $\lambda_{1}$ and $\lambda_{2}$ are corresponding Lagrange multipliers.

We note that values of the hyperparameters $\epsilon, \lambda_{1}$ and $\lambda_{2}$ are held fixed for all pairwise transport map calculations (E13, E14), (E14, E16), ... etc.

\section{Application of WOT to RGC diversification and long-range couplings}

We apply Waddington-OT to each pair of consecutive ages $t_{i}$ and $t_{i+1}$ to estimate the transport map $\widehat{\boldsymbol{\Pi}}_{t_{i}, t_{i+1}}$. Transport maps connecting non-consecutive time points $t_{i}$ and $t_{i+k}$ are estimated through a simple matrix multiplication of intermediate transport maps, 


$$
\widehat{\boldsymbol{\Pi}}_{t_{i}, t_{i+k}}=\widehat{\boldsymbol{\Pi}}_{t_{i}, t_{i+1}} \widehat{\boldsymbol{\Pi}}_{t_{i+1}, t_{i+2}} \cdots \widehat{\boldsymbol{\Pi}}_{t_{i+k-1}, t_{i+k}}
$$

1358

1359 The transport matrices $\widehat{\boldsymbol{\Pi}}_{t_{i}, t_{j}}$ encode fate relationships between cells at $t_{i}$ and cells at a 1360 later time $t_{j}>t_{i}$. These relationships can be analyzed at the level of clusters at $t_{j}$ to 1361 associate each cell $\boldsymbol{u} \in \mathcal{A}^{t_{i}}$ with transcriptomically defined cluster. This is particularly 1362 useful in estimating the terminal identity of immature RGCs.

1363

1364 Operationally we compute for each cell $\boldsymbol{u} \in \mathcal{A}^{t_{i}}$ a "cell fate vector" $f_{t_{j}}\left(\beta ; \boldsymbol{u}, t_{i}\right),(\beta=$ $13651,2, \ldots$ ) encoding the probabilities that $\boldsymbol{u}$ is associated with cluster $\mathcal{C}_{\beta}^{t_{j}}$ at time $t_{j}$, 1366

$$
f_{t_{j}}\left(\beta ; \boldsymbol{u}, t_{i}\right)=\frac{\sum_{\boldsymbol{v} \in \mathcal{C}_{\beta}^{t_{j}}} \widehat{\boldsymbol{\Pi}}_{t_{i}, t_{j}}(\boldsymbol{u}, \boldsymbol{v})}{\sum_{\beta} \sum_{\boldsymbol{v} \in \mathcal{C}_{\beta}^{t_{j}} \widehat{\boldsymbol{\Pi}}_{t_{i}, t_{j}}(\boldsymbol{u}, \boldsymbol{v})}}
$$

1367

1368 It is easy to verify that,

1369

$$
\sum_{\beta} f_{t_{j}}\left(\beta ; \boldsymbol{u}, t_{i}\right)=1 \forall \boldsymbol{u} \in \mathcal{A}^{t_{i}}
$$

1370

1371 The cell fate vector $f_{t_{j}}\left(\beta ; \boldsymbol{u}, t_{i}\right)$ encodes probabilistic associations between the cell $\boldsymbol{u}$ 1372 and terminal clusters at $t_{j}>t_{i}$ indexed by $\beta$. The "cluster ancestry vector" at an earlier 1373 time $t_{i}$ of a cluster $\mathcal{C}_{\beta}^{t_{j}}$ at time $t_{j}>t_{i}$, denoted $\Gamma_{t_{i}}\left(\boldsymbol{u} ; C_{\beta}^{t_{j}}\right)$, is defined as follows, 1374

$$
\Gamma_{t_{i}}\left(\boldsymbol{u} ; C_{\beta}^{t_{j}}\right)=\frac{\sum_{\boldsymbol{v} \in \mathcal{C}_{\beta}^{t_{j}}} \widehat{\boldsymbol{\Pi}}_{t_{i}, t_{j}}(\boldsymbol{u}, \boldsymbol{v})}{\sum_{\boldsymbol{u} \in \mathcal{A}^{t_{i}}} \sum_{\boldsymbol{v} \in \mathcal{C}_{\beta}^{t_{j}}} \widehat{\boldsymbol{\Pi}}_{t_{i}, t_{j}}(\boldsymbol{u}, \boldsymbol{v})}\left(t_{j}>t_{i}\right)
$$

1375 
1376 In a similar vein, the "cluster descendant vector" at a later time $t_{o}$ of a cluster $\mathcal{C}_{\beta}^{t_{j}}$ at a 1377 time $t_{j}<t_{o}$, denoted $\Gamma_{t_{o}}\left(\boldsymbol{u} ; C_{\beta}^{t_{j}}\right)$, is defined as,

1378

$$
\Gamma_{t_{o}}\left(\boldsymbol{u} ; C_{\beta}^{t_{j}}\right)=\frac{\sum_{\boldsymbol{v} \in \mathcal{C}_{\beta}^{t_{j}} \widehat{\boldsymbol{\Pi}}_{t_{j}, t_{o}}(\boldsymbol{v}, \boldsymbol{u})}}{\sum_{\boldsymbol{u} \in \mathcal{A} t_{o}} \sum_{\boldsymbol{v} \in \mathcal{C}_{\beta}^{t_{j}}} \widehat{\boldsymbol{\Pi}}_{t_{j}, t_{o}}(\boldsymbol{v}, \boldsymbol{u})}\left(t_{o}>t_{j}\right)
$$

1379

1380 These equations can be used to compute the putative ancestral or descendent cells 1381 associated with a cluster $C_{\beta}^{t_{j}}$ at time $t_{j}$.

1382

1383 Implementation details of WOT

1384

1385

RGC vectors from all ages were combined, median normalized and log-transformed. 1386 2854 HVGs were identified using the Gamma-Poisson model, and Waddington-OT was 1387 run on this matrix as follows,

1388

1389

wot optimal_transport --matrix RGC_mat.mtx --cell_days 1390 cell_day.txt --growth_iters 3 --epsilon 0.005 --out tmaps/RGC 1391

1392

Cell days were specified in cell_day.txt as $0,1,3,6,11$ and 20 for E13, E14, E16, 1393 P0, P5 and P56 respectively. We computed trajectories and fates for each age using 1394 the following command illustrated for P0,

1395

1396 wot trajectory --tmap tmaps/RGC --cell_set cell_sets.gmt --day 6 1397 --out tmaps/traj_RGC_PO.txt

Fates were computed as,

1400

1401

1402

wot fates --tmap tmaps/RGC --cell_set cell_sets.gmt --day 6 --out 1403 
1404 The above process was repeated for each age.

1405

1406 Multipotentiality of precursors

1407

1408 For each cell at ages E13-P5, we computed the terminal fate association $1409 f_{P 56}(\beta ; \boldsymbol{u}, t), t \in(E 13, E 14, \ldots, P 5)$, quantifying the probability that it is a precursor of type $1410 \beta \in(1,2, \ldots 45)$. Note that $f_{P 56}(\beta ; \boldsymbol{u}, t)$ is denoted as $f_{\beta}$ for brevity in the main text. We 1411 define,

1412

$$
P(\boldsymbol{u} ; t)=\frac{1}{\sum_{\beta} f_{P 56}(\beta ; \boldsymbol{u}, t)^{2}}
$$

1413

1414 as the potential of precursor $\boldsymbol{u}$ at age $t$. Values of $P$ range between 1 and 45 , with lower 1415 values indicating restriction of fate and higher values suggesting multipotentiality.

\section{Network analysis of fate couplings}

1423

1424 We define,

1425

$$
\begin{aligned}
& C(\alpha, \beta ; t) \\
& =\frac{\frac{1}{\left|\mathcal{A}^{t}\right|} \sum_{\boldsymbol{u} \in \mathcal{A}^{t}}\left(f_{P 56}(\alpha ; \boldsymbol{u}, t)-\overline{f_{P 56}(\alpha ; t)}\right)\left(f_{P 56}(\beta ; \boldsymbol{u}, t)-\overline{f_{P 56}(\beta ; t)}\right)}{\sqrt{\frac{1}{\left|\mathcal{A}^{t}\right|} \sum_{\boldsymbol{u} \in \mathcal{A}^{t}}\left(f_{P 56}(\alpha ; \boldsymbol{u}, t)-\overline{f_{P 56}(\alpha ; t)}\right)^{2}} \sqrt{\frac{1}{\left|\mathcal{A}^{t}\right|} \sum_{v \in \mathcal{A}^{t}}\left(f_{P 56}(\beta ; \boldsymbol{v}, t)-\overline{f_{P 56}(\alpha ; t)}\right)}}
\end{aligned}
$$


1427 as the fate coupling between RGC types $\alpha$ and $\beta$ at age $t$. Clearly, $C(\alpha, \beta ; t)$ is simply 1428 the Pearson correlation coefficient between $f_{P 56}(\alpha ; \boldsymbol{u}, t)$ and $f_{P 56}(\beta ; \boldsymbol{u}, t)$, the 1429 probabilities that a cell $\boldsymbol{u} \in \mathcal{A}^{t}$ is a precursor of $\alpha$ and $\beta$ precursor. Here, 1430

$$
f_{P 56}(\alpha ; t)=\frac{1}{\left|\mathcal{A}^{t}\right|} \sum_{\boldsymbol{u} \in \mathcal{A}^{t}} f_{P 56}(\alpha ; \boldsymbol{u}, t)
$$

1431

1432 is the mean probability that a cell at age $t$ is a precursor of type $\alpha$. We computed $1433 C(\alpha, \beta ; t)$ across all 990 pairs of RGC types at each immature age $t \in$ $1434(E 13, E 14, \ldots, P 5)$. The values $C(\alpha, \beta ; E 13)$ were used as edge weights to visualize the 1435 fate coupling network of RGC types using the force-directed layout method 1436 (Fruchterman \& Reingold, 1991) as implemented in the R package igraph. The node 1437 layout was computed using $C(\alpha, \beta ; E 13)$ values. For other ages, the node layout at E13 1438 was retained but the edges were replotted based on $C(\alpha, \beta ; t)$ values at the 1439 corresponding age.

1441 We computed a null distribution of $C(\alpha, \beta ; t)$ by randomizing the values of $f_{P 56}(\alpha ; \boldsymbol{u}, t)$ 1442 within each cell $\boldsymbol{u}$ across types. Null values of $C(\alpha, \beta ; t)$ rarely exceeded 0.1 and never 1443 exceeded 0.2, so only edges with larger weights were visualized in Figure 5.

\section{Decay of pairwise couplings}

1447 For each pair of RGC types $\alpha$ and $\beta$, we fitted a logistic equation to model the decay of 1448 pairwise couplings as, 1449

$$
C(\alpha, \beta ; t)=\frac{1}{1+\exp \left(\beta_{0}+\beta_{1} t\right)}
$$

1451 The values of $t$ corresponding to E13, E14, E16, P0 and P5 were $t=0,1,3,6,11$, with $1452 C(\alpha, \beta ; t)$ computed as above. We also assumed that $C(\alpha, \beta ; t)=0$ at $\mathrm{t}=36$, 1453 corresponding to P30. Thus, six data-points were used to estimate two parameters for 
1454 each of the 180 pairs of RGC types that had non-zero values of $C(\alpha, \beta ; t)$. The nls 1455 function from the $R$ package stats was used to estimate $\beta_{0}$ and $\beta_{1}$. The results are 1456 plotted in Figure $\mathbf{5 f}$.

\section{Logistic modeling of specification and calculation of $\tau_{s p}$}

We hypothesized that the specification of a type $\beta$ corresponds to the localization of its precursors in transcriptomic space. The extent of localization for a RGC type $\beta$ at across the time course was calculated as follows. At each age $t$, we identified the set of precursor RGCs $\operatorname{Prec}(\beta ; t)$ showing the highest fate probability corresponding to type $\beta$, 1464

$$
\operatorname{Prec}(\beta ; t)=\left\{\boldsymbol{u} \in \mathcal{A}^{t} \mid f_{P 56}(\beta ; \boldsymbol{u}, t)>f_{P 56}(\alpha \neq \beta ; \boldsymbol{u}, t)\right\}
$$

1465

1466 Next, we calculated how the precursors of $\beta$ were distributed across clusters at time $t$. 1467 We computed the occupancy fractions of precursor cells for type $\beta$ across all cluster $1468 \mathcal{C}_{k}, k=1,2, \ldots, N(t)$ at a particular time $t(N(t)$ is the number of transcriptomically 1469 defined clusters at time $t$ )

1470

$$
p_{k}(\beta ; t)=\frac{\#\left\{\text { cluster }(\boldsymbol{u})=\mathcal{C}_{k} \forall \boldsymbol{u} \in \operatorname{Prec}(\beta ; t)\right\}}{\#\{\boldsymbol{u} \in \operatorname{Prec}(\beta ; t)\}}
$$

1472 The localization score for each type $\beta$ at a given time $t$ was defined as, 1473

$$
\operatorname{Localization}(\beta ; t)=1-\frac{\sum_{k=1}^{N(t)} \frac{1}{p_{k}(\beta ; t)^{2}}}{N(t)}
$$

1474 where the index $k$ ranges over the number of clusters at time $t$. As defined 1475 Localization $(\beta ; t)$ is restricted to be between 0 to 1 , with higher values representing a 1476 greater specification. We used a logistic model to approximate the localization of each 1477 type as, 


$$
\operatorname{Localization}(\beta ; t)=\frac{\exp \left(\gamma_{0}+\gamma_{1} t\right)}{1+\exp \left(\gamma_{0}+\gamma_{1} t\right)}
$$

1479 As in the previous section the $\mathrm{n}$ ls function was used to estimate the logistic parameters $1480 \gamma_{0}$ and $\gamma_{1}$. We consider a type $\beta$ as specified if its specification crosses the line $y(t)=$ $14810.95(1-1 / N(t))$. Thus the specification time for a type $\beta$ is defined as,

$$
\tau_{s p}(\beta)=\operatorname{argmin}_{t} \text { Localization }_{(\beta ; t)} \geq y(t)
$$

1484 Note that as defined, $\tau_{s p}$ can be any time point in the interval (E13, P30) corresponding 1485 to $t \in(0,36)$.

\section{Inference of laterality in RGC types}

To identify putative ipsilateral and contralateral specified-RGC precursors at E13, we scored each precursor RGC based on their expression of bonafide ipsilateral genes Zic2, Zic1 and Igfbp5) and bonafide contralateral genes (Is/2, Fgf12, Igf1) as in (Wang et al., 2016). We refer to these as I-RGC and C-RGC scores. Putative I-RGCs were those cells that expressed the I-RGC score at 1.5 standard deviations higher than the 1494 mean across all cells, and those that express the C-RGC score at 1.5 standard 1495 deviations lower than the mean across all cells. C-RGCs were defined analogously. 1496 Many cells did not express either of these marker sets as shown in Figure 7c. These 1497 are likely to be RGCs that have not declared their laterality, or C-RGCs that are not 1498 defined by the expression of Is/2, Fgf12, and Igf1.

WOT was then used to compute the descendants of E13 I-RGCs at all subsequent ages through P56 using the wot fates command introduced above. These descendants were used for two purposes. First, we assessed the proportion of putative I-RGCs

1503 across types as in Figure 7d. We also performed a differential gene expression test 1504 between putative I-RGCs and the remaining RGCs at all ages, as shown in Figures. 1505 7e,f and Figure 7-figure supplement 1d,e. 


\section{Comparison with previous retina scRNA-seq datasets}

1508

We compared our data with two scRNA-seq studies that profiled the whole retina during 1510 development:

1. (Clark et al., 2019): Count matrices and cell/gene level annotations were downloaded from the author's public repository https://github.com/gofflab/developing_mouse_retina_scRNASeq. This dataset contains whole retinal cells sampled at 10 time points $(E 11, E 12, E 14, E 16, E 18$, P0, P2, P5, P8, P14) with four of these (E14, E16, P0, P5) common with our study. We excluded P5 from our analysis as only $\mathrm{N}=11 \mathrm{RGCs}$ were identified by the authors at this time point.

2. (Lo Giudice et al., 2019): Count matrix corresponding to E15.5 retinal cells was kindly provided by the authors.

3. (Rheaume et al., 2018): Count matrix corresponding to P5 RGCs was downloaded from the online submission.

For consistency with our filtering parameters, we extracted cells based on a cutoff of 700 genes/cell from each of the above datasets. For the Clark et al. dataset, this selected 17,827 cells at E14, 1,674 cells at E16 and 8,343 cells at P0 respectively ( $\mathrm{N}=27,844$ cells). In these samples, RGCs comprised $191 \%, 281 \%$ and $0.451 \%$. For the Giudice et al. dataset, this selected 5218 cells, of which $231 \%$ were RGCs. II

The Rheaume et al. dataset was directly compared with P5 RGCs collected in this study using supervised classification (Figure 1-figure supplement 1i). Clark et al. and Lo Giudice et al. data were combined with the retinal cells profiled in this study at corresponding time points (25,685 cells at E14; 21,274 cells at E16; and 23,251 cells at P0). Together this resulted in a 14,350 genes $X 103,272$ cells expression matrix that was analyzed following steps outlined previously. In the alignment step, cells from each combination of age and study was considered as a separate "batch". We visualized the 
1538 transcriptional heterogeneity of the full dataset using UMAP, and used the expression of 1539 canonical markers to confirm the co-clustering of cell-classes in Figure 1-figure 1540 supplement 1 (Rbpms for RGCs, Tfap2b for ACs, Fgf15 for RPCs and Gngt2 for 1541 RPCs).

\section{Acknowledgments}

1544 Funding for this study was provided by National Institute of Health grants 1545 R37NS029169, R01EY022073 (JRS), NSF GRP DGE1752814 (SB), and 1546 R00EY028625 (KS), and startup funding from UC Berkeley (KS, SB). We are grateful to 1547 Prof. Seth Blackshaw for helpful suggestions on the preprint. The authors would like to 1548 thank Joshua Hahn for valuable feedback.

1550 Competing interests

1551 The authors declare no competing interests.

Correspondence and requests for materials should be addressed to K.S. or J.R.S.

1556 All scRNA-seq data collected in this study are submitted to the Gene Expression 1557 Omnibus (GEO) under GSE185671. The data can be visualized on the Broad Institute's 1558 Single Cell Portal under the identifier SCP1706.

1559

1560

1561

1562

\section{Code availability}

The scripts (written in R) generated for this study are shared at

\section{Figure Legends}

Figure 1, Transcriptomic profiling of single postmitotic RGCs during embryonic and postnatal development in mice. 
1571 Müller glia (MGs) and retinal ganglion cells (RGCs). PRs reside in the outer nuclear 1572 layer (ONL), while BCs, HCs and most ACs reside in the inner nuclear layer (INL). 1573 RGCs and some ACs reside in the ganglion cell layer (GCL). Axons of RGCs project to higher visual areas via the optic nerve.

b. Retinal section of the indicated ages labeled for the cell-cycle marker MKI67 (red) and the RGC marker RBPMS (green); nuclei are counterstained by the Hoeschst dye (blue). Micrographs are orientated as the schematic in panel a.

c. Visualization of transcriptional diversity of 98,452 cells using Uniform Manifold Approximation and Projection (UMAP), a nonlinear dimensionality reduction algorithm that assigns proximal $x-y$ coordinates to cells (dots) with similar transcriptional profiles(Becht et al., 2019).

d. Same as c, with cells colored by cell class, assigned based on transcriptional signatures displayed in panel e. RPC, retinal progenitor cells; Ant. Seg., anterior segment cells.

e. Tracksplot showing expression patterns of cell-class specific marker genes (rows) across single cells (columns). Cells are grouped by class as in d. For each class, we randomly sampled $20 \%$ of total cells covering all immature time points (E13, E14, E16, P0, P5). For each gene, the scale on the y-axis (right) corresponds to normalized, logtransformed transcript counts detected in each cell.

\section{Figure 2. The number and discreteness of transcriptomic clusters of RGCs} increases with age.

a. Extreme models of RGC diversification. In one scenario (left) immature RGCs commit to one of the terminal types by the time of birth (i.e. mitotic exit) or shortly after. Alternatively (right), initially identical postmitotic RGC precursors acquire distinct molecular identities in a gradual process of restriction.

b-g. Visualization of transcriptomic diversity of immature RGCs at E13 (b), E14 (c), E16 (d), P0 (e), P5 (f) and P56 (g) using UMAP. Cells are colored by their cluster identity, determined independently using dimensionality reduction and graph clustering (Methods). Clusters are numbered based on decreasing size at each age. Data for adults (P56) are replotted from ref. 10. In that study 45 transcriptomic types were identified via unsupervised approaches, one of which was mapped to 2 known functional types by supervised approaches. We do not distinguish them in this study.

h. Transcriptional diversity of RGCs as measured by the Rao diversity index (y-axis) increases with age (x-axis). The trend is insensitive to the number of genes used to compute inter-cluster distance (colors). See Methods for details underlying the calculation.

i. Transcriptomic distinctions between RGC clusters become sharper with age as shown by decreasing average per-cluster error of a multiclass-classifier with age. Gradient boosted decision trees were trained on a subset of the data, and applied on held out samples to determine the test error.

j. RGC clusters also become better separated in the UMAP embedding, as shown by decreasing values of the average relative cluster diameter with age.

Figure 3. Incompletely specified temporal relationships among RGC clusters. 
a. Top: Specified (left) and non-specified (right) modes of diversification. Nodes denote transcriptomic clusters of immature RGCs, and arrows denote fate relationships. Bottom: Confusion matrices depicting transcriptomic correspondence between late and early clusters expected for the two modes. Circles and colors indicate the percentage of a given late cluster (row) assigned to a corresponding early cluster (column) by transcriptome-based classifier trained on early clusters. The number of late and early clusters have been set to eight and four for illustration purposes.

b. Barplot showing values of the normalized conditional entropy (NCE) for each age calculated using the transcriptional cluster IDs and the Xgboost-assigned cluster IDs corresponding to the next age or to P56 (E.g. for E13, the NCE was calculated across E13 RGCs by comparing their transcriptional cluster ID with assigned E14 cluster IDs based on a classifier trained on the E14 data). Lower values indicate specific mappings. c. Same as b, but plotting values of the adjusted Rand Index (ARI), where larger values correspond to higher specificity. d-h. Confusion matrices (representation as in a), showing transcriptomic correspondence between consecutive ages: E14-E13 (d), E16-E14 (e), P0-E16 (f), P5P0 (g), P56-P5 (h). In each case, the classifier was trained on the late time point and applied to the early time point. Rows sum to $100 \%$.

\section{Figure 4. Multipotential fate associations between immature RGCs and terminal types inferred via Optimal Transport}

a. Extreme models of diversification at single-cell resolution. Multipotential fate associations in a transcriptionally defined cluster (ellipse) could arise from a mixture of unipotential RGCs (left) or from multipotential RGCs (right).

b. Distributions of potential $P$ across immature RGCs by age showing that restriction increases with age.

c. Inter- and intra-cluster variation of potential by age. At each age, variation in the potential values are shown for each transcriptomically defined cluster at that age. Dots denote the average potential and dotted lines depict the standard deviation for cells within each cluster.

d-h. UMAP projections of E13 (d), E14 (e), E16 (f), P0 (g) and P5 (h) RGCs as in Fig. 2, but with individual cells colored by their inferred potential. Potential of all RGCs at P56 $=1$. The colorbar on the lower right is common to all panels, and values are thresholded at $P=20$.

1661

\section{Figure 5. Fate decoupling of RGC types}

a. Force-directed layout visualization of fate couplings at E13, with nodes representing RGC types (numbered as in Tran, 2019) and the thickness of edges representing values of $C(l, m ; E 13)$. Edges with $C(I, m ; E 13)<0.2$ are not shown. Number of edges with $C(I, m ; E 13)>0.2$ are indicated on top.

b-e. Visualization of fate couplings at E14 (B), E16 (C), P0 (D) and P5 (E). The positions of the nodes are maintained as in panel $\mathrm{a}$, but the edges are redrawn based on values of $C(l, m$;age) at each age. As in panel a, we only show edges $C(I, m$; age $)>0.2$. 
$f$. The decay of pairwise fate couplings ( $y$-axis) with age ( $x$-axis). Each line corresponds shows the temporal decay of $C(I, m)$ for RGC pair I and $m$ estimated via a logistic model (Methods). For each pair, couplings at each age were fit to a model $C(l, m$; age $)=$ $1 /\left(1+e^{\beta_{0}+\beta_{1} * a g e}\right)$ with $\beta_{0}, \beta_{1}$ representing fitted parameters. The fitting was performed using data for ages E13, E14, E16, P0 and P5. The shaded portions correspond to the periods E8-E13 and P5- represent extrapolations of the model. Black lines highlight the decay of all non-zero pairwise couplings for RGC type $C 8$ as an example. g. Schematic showing logistic modeling to estimate specification time $\tau_{s p}$ for a particular type. The $y$-axis is a measure of the extent to which precursors biased towards the type are present in a single transcriptomically defined cluster (i.e. localization, see Methods for details). Localization is defined as a numerical value in the range $(0,1)$ with higher values consistent with increasing specification. Individual triangles represent the localization values computed using WOT inferred fate couplings at each age, while the curve represents the fit using the logistic model. Dotted line shows the minimum threshold a type to be specified at each age. Its curved shape arises due to the increase in the number of clusters with age. h. Localization curves (as in panel g) for the 38 RGC types showing the range of inferred specification times. 7 low frequency types have been excluded from display

\section{Figure 6. Temporal dynamics of RGC subsets expressing specific TFs} their selective expression of TFs at P56. Asterisks denote 3/45 types that express more than 1 TF (also see Figure 6-figure supplement 1a).

1692 b. Box-and-whisker plots showing that pairwise fate couplings are higher between types within the same TF subclass than between types in different TF subclasses at all immature ages. Black horizontal line, median; bars, interquartile range; vertical lines, $1^{\text {st }}$ and $99^{\text {th }}$ percentile; dots, outliers. Stars indicate significant $p$-values based on a twosided t-test $\left({ }^{* * * *}, \mathrm{p}<10^{-7} ;{ }^{* * *}, \mathrm{p}<10^{-5} ;{ }^{* *}, \mathrm{p}<10^{-2}\right)$.

c. Eomes+ types. Top: UMAP representation of E13 RGCs with cells colored based on their cumulative fate association towards the 7 Eomes+ types. Bottom: UMAP representation of P5 RGCs with cells colored based on their cumulative fate association towards the 7 Eomes+ types. The value corresponding to the color of each cell (colorbar, right) can be interpreted as the probability of commitment towards the corresponding subclass.

d. Same as c for Mafb+ types

e. Same as c for Neurod2+ types

f-h. Localization curves (as in Fig. 5g) for Eomes+ types (f), Mafb+ types (g) and on top of each panel. 
Figure 7. Transcriptomic identification of ipsilaterally projecting RGCs

a. Zic2, an I-RGC marker and Is/2, a C-RGC marker, are expressed in a mutually exclusive pattern at E13 (left), E14 (middle) and E16 (right). Zic2 is undetectable after E16 (Figure 7-figure supplement 1a). Cells are colored based on a bivariate color scale representing co-expression of two markers (colorbar, right). b. Zic2 and Igfbp5, two I-RGC markers, are co-expressed at E13 (left) and E14 (middle). Representation as in panel a.

c. Scatter-plot of gene signatures used to identify I-RGCs (y-axis) and C-RGCs (x-axis) at E13 are negatively correlated (Pearson $R=-0.61$ ). Each dot corresponds to a cell, the color represents the number of cells located at a particular $(x, y)$ location (see colorbar, right).

d. Barplot showing \% of putative I-RGCs (y-axis) within each of the 45 adult RGC types, estimated by computing the descendants of E13 I-RGCs using WOT. RGC types are arranged along the $x$-axis based on their membership of TF-groups shown in Fig. 6a (annotation matrix, bottom).

e. Volcano plot showing DE genes (MAST test, $p<10^{-6}$ ) between predicted I-RGCs and $\mathrm{C}$-RGCs at E13. The $x$ - and the $y$-axes show the fold-change and the $p$-value in log2and $\log 10-$ units, respectively. Dots represent genes, with red and blue dots highlighting I- and C-RGC enriched genes respectively at fold-change $>1.5$ and Bonferroni corrected $p$-value $<5 \times 10^{-5}$. The two vertical bars correspond to a fold-change of 1.5 in either direction. Select I-RGC and C-RGC enriched genes are labeled. f. Same as panel e, for E14.

Figure 1-figure supplement 1. Separation of major transcriptomic groups and assessment of immature RGCs at E13-P5.

a. Retinal sections stained with L1CAM (red), which was used to enrich for RGCs at early stages, and the pan-RGC marker RBPMS, (green) at E13, E14, E16 and P0. Nuclei are counterstained by the Hoeschst dye (blue).

b. Relative proportions (y-axis) of major cell classes shown in Fig. 1c-e at each combination of age and enrichment method. Both anti-Thy 1 and anti-L1cam were used to enrich RGCs at E13, E14, E16 and P0, but only anti-Thy1 was used at P5, because L1cam becomes localized to axons postnatally. AC, Amacrine Cells; RPC, retinal progenitor cells.

c. Box and whisker plots show gene expression levels of key markers by RGCs as a function of age and enrichment method. Markers shown shown are two pan-RGC markers, Rbpms, Nefl, and the two cell-surface proteins used for enrichment, Thy 1 and L1cam. Note that Thy1 expression is poor at E13, consistent with low RGC yield in antiThy1 enriched cells (panel B). Black horizontal line, median; bars, interquartile range; vertical lines, range; dots, outliers.

d. Dotplot showing genes (columns) that are selectively expressed in RGCs and RPCs. The size of each circle is proportional to the percentage of cells expressing the gene, and the color depicts the average log-normalized expression. 
e. Co-embedding analysis of E14, E16 and P0 data collected in this study with whole retina single-cell transcriptomes in independent studies: E14, E16 and P0 data from(Clark et al., 2019) and E15.5 data from(Lo Giudice et al., 2019). Cells (points) are visualized in UMAP and colored by study of origin.

1757 f. Same as e, with cells colored by the expression level of Nefl, an RGC marker. This shows the higher enrichment of RGCs in our study compared to(Clark et al., 2019) and(Lo Giudice et al., 2019). g. Same as d, with cells colored by expression level of Fgf15, an RPC marker.

h. Relative proportions of major cell classes across different datasets analyzed in panel e separated by age. T, this study.

i. Confusion matrix showing post hoc transcriptomic correspondence between P5 RGC types identified in Rheaume et al., 2018 (y-axis) and P5 RGC clusters reported in this study (x-axis). In each case, RGCs from Rheaume et al. were assigned P5 cluster labels using an Xgboost classifier trained on P5 RGCs profiled in this study.

Figure 2-figure supplement 1. Transcriptomic diversity of immature RGCs by age. a-c. UMAP embedding for RGCs at E14 (a, same as Fig. 2c), E16 (b, same as Fig. 2d) and PO (c, same as Fig. 2e) with cells colored by enrichment method showing comparable transcriptomic diversity of immature RGCs enriched by L1cam or Thy1. All clusters are present in both collection types, although the relative frequencies vary. This concordance supports the idea that neither marker excludes particular types.

d. Simpson and Shannon diversity indices (see Methods) associated with clustering decrease and increase with age respectively, consistent with increasing transcriptomic diversity.

Figure 3-figure supplement 1. Temporal correspondences between transcriptomic clusters evaluated using supervised classification.

a-d. Confusion matrices, showing transcriptomic correspondence between adult RGC types at P56 (rows) and immature RGC clusters (columns) at ages E13 (a), E14 (b), E16 (c) and P0 (d). In each case, immature RGCs were assigned adult labels using an Xgboost classifier trained on adult RGCs. The P56 to P5 mapping is shown in Fig. $\mathbf{2 h}$. e. Line plots showing occupancy fraction (OF) of mapping of an early cluster to cluster IDs at later ages. OF values quantifies the specificity of mapping of an early cluster to late clusters, with lower values denoting higher specificity. The average occupancy fraction across clusters decreases steadily with age consistent with the decrease and increase in NCE and ARI respectively (Figs. 3b,c). Error bars indicate standard deviation computed across clusters. Also, as expected, the occupancy fraction values are lower for mapping to adjacent time points than to P56.

Figure 4-figure supplement 1. Variations in WOT inferred temporal couplings and tests across variations in hyperparameters.

a. Variations in WOT inferred temporal couplings $\left(\Pi_{i j}\right)$ at the level of cells and clusters to changes in the set of highly variable genes (HVGs) used for computing transport maps. Four sets of features were tested corresponding to the top 800, 1100, 1400 and 1800 HVGs based on our previously described Poisson-Gamma model (Pandey et al., 
2018). Using these sets, we inferred four corresponding transport maps at each of the 5 age pairs E13-E14, E14-E16, E16-P0, P0-P5 and P5-P56. The entropic regularization hyperparameter $\epsilon$ (see panels $\mathrm{b}, \mathrm{c}$ ) was held constant at a value $2^{-7}$ in these tests. At each age pair, we computed the Pearson correlation coefficient (PCC) between estimated temporal couplings for every older cell (column of the transport map $\Pi$ ) across each pairwise combination of the four transport maps, towards a total of 6 combinations. These are indicated as red dots and lines (mean \pm SD). We then grouped (summed) the rows of the transport map by transcriptomic cluster at the younger age, such that each element of the new matrix indicates cell (column)-cluster (row) couplings. The PCC of these couplings were computed for each older cell (column) within each pairwise combination of the four transport maps and are indicated as green dots and lines (mean \pm SD). Finally, we grouped (summed) both the rows and columns of the transport map by transcriptomic cluster at either age to obtain a matrix of cluster-cluster couplings. The PCC values of these couplings were computed for each older cluster within each pairwise combination of the four transport maps and are indicated as blue dots and lines (mean $\pm S D$ ). We find that the cell-cell couplings increase in robustness at later ages, but the cell-cluster and cluster-cluster couplings are quite robust (correlation $>0.6$ ).

b. Variations in WOT inferred temporal couplings at the level of cells and clusters as in panel $A$, but to changes in the entropic regularization $\epsilon$. Six values were used $-\left(2^{-8}, 2^{-7}\right.$ $, 2^{-6}, 2^{-5}, 2^{-4}, 2^{-3}$ ) with increasing values corresponding to more transport maps with decreasingly localized (or increasingly distributed) couplings. At each age pair, 6 transport maps are computed and PCC values for cell-cell, cell-cluster and clustercluster couplings are computed as in panel A for each of 15 transport map pairs. Here too, the cluster-cluster and cell-cluster couplings show higher stability, although at later stages higher values of $\epsilon$ exhibit loss of stability even at the cluster-cluster level (see panel c).

C. Heatmap showing cluster-cluster PCC values for P5-P56 transport maps inferred using different values of the entropic regularization parameter, epsilon (rows and columns). Loss of stability occurs at higher values of the entropic regularization, consistent with panel B. Based on this we used epsilon $=2^{-7}$ to calculate results shown in Fig. 4.

\section{Figure 4-figure supplement 2. Temporal correspondences between transcriptomic clusters evaluated using Waddington Optimal Transport.}

a-e. Average temporal couplings at the level of clusters. Panels correspond to the pairs E14-E13 (a), E16-E14 (b), P0-E16 (c), P5-P0 (d), P56-P5 (e), respectively. In each case, the WOT inferred transport map was grouped along rows and columns based on transcriptomic cluster, and the elements were summed within each group. The resulting matrix was normalized such that each row sums to $100 \%$. These matrices strongly resemble those in Figs. 3d-h, as confirmed by the high values of the Pearson correlation coefficient (top, all $p \geq 0.92$ ). 
Figure 5-figure supplement 1. Fate decoupling and temporal specification of RGC types.

a-c. Examples of fate decoupling. Panels from left to right correspond to ages E13-P5 with precursor RGCs shown on a reduced dimensional UMAP representation as in Figs. 2c-g. Each RGC is colored using a biaxial color scale (legend) based on its predicted fate values. C12 and C22 are transcriptomically distinct as early as E13 (a). C19 and C20 exhibit high fate coupling at all embryonic ages and are only decoupled at P5 (b). C21 and C34 decouple around E16 (c).

d. Same as Fig. 6h showing specification curves for RGC types, but in this case each curve is colored based on adult frequency (colorbar, right). The 7 curves marked by

Figure 6-figure supplement 1. Transcription factor (TF) based subgroups.

a. Dotplot showing mutually exclusive patterns of expression of TFs that mark groups of RGC types. In addition to the 8 TFs shown in Fig. 6a, we highlight Zic1, which selectively labels C6. Selectively expressed TFs could not be identified for 4 types (C1, C2, C11, and C15).

b-e. Localization curves (as in Fig. 5g) for Tbr1+ types (b), Tfap2d+ types (c), Foxp2+ types (d) and Bnc2+ types (e), Note that the "low frequency" types labeled in Figure 5figure supplement $1 \mathbf{d}$ are not shown. The mean specification time $\tau_{s p}$ for each group is shown above the graphs.

f. Correlation of fate coupling at E13 with transcriptomic correlation at P56.

g. Same as Fig. 6a, with nodes corresponding to subclasses defined in Tran et al., 2019, which includes ipRGCs, alpha-RGCs and T5-RGCs.

Figure 7-figure supplement 1. Transcriptomic analysis of RGC laterality.

a. RGCs at E13, E14, E16, P0, and P5 colored by their expression of Zic2, an I-RGC marker. Zic2 is expressed in a localized fashion until E16 and becomes undetectable beyond this age.

b. Zic2, an I-RGC marker and Igf1, a C-RGC marker, are expressed in mutually exclusive patterns at E13-E16.

c. Zic2 and Zic1, two I-RGC markers, are co-expressed in subsets of RGCs at E13-E16. d. Volcano plot showing DE genes (MAST test, $\mathrm{p}<10^{-6}$ ) between predicted I-RGCs and C-RGCs at E16. The $x$ - and the y-axes show the fold-change and the $p$-value in log2and $\log 10$ - units, respectively. Dots represent genes, with red and dots highlighting Iand C-RGC enriched genes respectively at fold-change $>1.5$ and Bonferroni corrected $\mathrm{p}$-value $<5 \mathrm{e}-5$. The two vertical bars correspond to a fold-change of 1.5 in either direction.

1889 


\section{References}

Baden, T., Berens, P., Franke, K., Roman Roson, M., Bethge, M., \& Euler, T. (2016). The functional diversity of retinal ganglion cells in the mouse. Nature, 529(7586), 345-350. doi:10.1038/nature16468

Baden, T., Euler, T., \& Berens, P. (2020). Understanding the retinal basis of vision across species. Nat Rev Neurosci, 21(1), 5-20. doi:10.1038/s41583-019-0242-1

1903

1904

1905

1906

1907

1908

1909

1910

1911

1912

1913

1914

1915

1916

Bae, J. A., Mu, S., Kim, J. S., Turner, N. L., Tartavull, I., Kemnitz, N., . . Eyewirers. (2018). Digital Museum of Retinal Ganglion Cells with Dense Anatomy and Physiology. Cell, 173(5), 1293-1306 e1219. doi:10.1016/j.cell.2018.04.040

Baron, C. S., \& van Oudenaarden, A. (2019). Unravelling cellular relationships during development and regeneration using genetic lineage tracing. Nat Rev Mol Cell Biol, 20(12), 753-765. doi:10.1038/s41580-019-0186-3

Barres, B. A., Silverstein, B. E., Corey, D. P., \& Chun, L. L. (1988). Immunological, morphological, and electrophysiological variation among retinal ganglion cells purified by panning. Neuron, 1(9), 791-803. doi:10.1016/0896-6273(88)90127-4

Bassett, E. A., \& Wallace, V. A. (2012). Cell fate determination in the vertebrate retina. Trends Neurosci, 35(9), 565-573. doi:10.1016/j.tins.2012.05.004

Becht, E., Mclnnes, L., Healy, J., Dutertre, C.-A., Kwok, I. W., Ng, L. G., . . Newell, E. W. (2019). Dimensionality reduction for visualizing single-cell data using UMAP. Nature biotechnology, 37(1), 38-44.

Berson, D. M., Dunn, F. A., \& Takao, M. (2002). Phototransduction by retinal ganglion cells that set the circadian clock. Science, 295(5557), 1070-1073. doi:10.1126/science.1067262

Blackshaw, S., Harpavat, S., Trimarchi, J., Cai, L., Huang, H., Kuo, W. P., . . Cepko, C. L. (2004). Genomic analysis of mouse retinal development. PLoS Biol, 2(9), E247. doi:10.1371/journal.pbio.0020247

Boije, H., MacDonald, R. B., \& Harris, W. A. (2014). Reconciling competence and transcriptional hierarchies with stochasticity in retinal lineages. Curr Opin Neurobiol, 27, 68-74. doi:10.1016/j.conb.2014.02.014 
1925

1926

1927

1928

1929

1930

1931

1932

1933

1934

1935

1936

1937

1938

1939

1940

1941

1942

1943

1944

1945

1946

1947

1948

1949

1950

1951

1952

1953

1954

1955

1956

1957

1958

Brown, N. L., Patel, S., Brzezinski, J., \& Glaser, T. (2001). Math5 is required for retinal ganglion cell and optic nerve formation. Development, 128(13), 2497-2508. Retrieved from https://www.ncbi.nlm.nih.gov/pubmed/11493566

Cembrowski, M. S., \& Spruston, N. (2019). Heterogeneity within classical cell types is the rule: lessons from hippocampal pyramidal neurons. Nat Rev Neurosci, 20(4), 193-204. doi:10.1038/s41583-019-0125-5

Cepko, C. (2014). Intrinsically different retinal progenitor cells produce specific types of progeny. Nat Rev Neurosci, 15(9), 615-627. doi:10.1038/nrn3767

Chen, S., Wang, Q. L., Nie, Z., Sun, H., Lennon, G., Copeland, N. G., . . Zack, D. J. (1997). Crx, a novel Otx-like paired-homeodomain protein, binds to and transactivates photoreceptor cell-specific genes. Neuron, 19(5), 1017-1030. doi:10.1016/s08966273(00)80394-3

Chen, T., \& Guestrin, C. (2016). Xgboost: A scalable tree boosting system. Paper presented at the Proceedings of the 22nd acm sigkdd international conference on knowledge discovery and data mining.

Chizat, L., Peyré, G., Schmitzer, B., \& Vialard, F.-X. (2018). Scaling algorithms for unbalanced optimal transport problems. Mathematics of Computation, 87(314), 2563-2609.

Clark, B. S., Stein-O'Brien, G. L., Shiau, F., Cannon, G. H., Davis-Marcisak, E., Sherman, T., . . . Blackshaw, S. (2019). Single-Cell RNA-Seq Analysis of Retinal Development Identifies NFI Factors as Regulating Mitotic Exit and Late-Born Cell Specification. Neuron, 102(6), 1111-1126 e1115. doi:10.1016/j.neuron.2019.04.010

Demyanenko, G. P., \& Maness, P. F. (2003). The L1 cell adhesion molecule is essential for topographic mapping of retinal axons. J Neurosci, 23(2), 530-538. Retrieved from https://www.ncbi.nlm.nih.gov/pubmed/12533613

Di Bella, D. J., Habibi, E., Stickels, R. R., Scalia, G., Brown, J., Yadollahpour, P., . . Arlotta, P. (2021). Molecular logic of cellular diversification in the mouse cerebral cortex. Nature, 595(7868), 554-559. doi:10.1038/s41586-021-03670-5

Dowling, J. E. (2012). The Retina: An Approachable Part of the Brain (2nd ed.): Harvard University Press.

Drager, U. C. (1985). Birth dates of retinal ganglion cells giving rise to the crossed and uncrossed optic projections in the mouse. Proc R Soc Lond B Biol Sci, 224(1234), 5777. doi:10.1098/rspb.1985.0021

Espinosa-Medina, I., Garcia-Marques, J., Cepko, C., \& Lee, T. (2019). High-throughput dense reconstruction of cell lineages. Open Biol, 9(12), 190229. doi:10.1098/rsob.190229 
1959

1960

1961

1962

1963

1964

1965

1966

1967

1968

1969

1970

1971

1972

1973

1974

1975

1976

1977

1978

1979

1980

1981

1982

1983

1984

1985

1986

1987

1988

1989

1990

1991

1992

Famiglietti, E. V., Jr., \& Kolb, H. (1976). Structural basis for ON-and OFF-center responses in retinal ganglion cells. Science, 194(4261), 193-195. doi:10.1126/science.959847

Farah, M. H., \& Easter, S. S., Jr. (2005). Cell birth and death in the mouse retinal ganglion cell layer. J Comp Neurol, 489(1), 120-134. doi:10.1002/cne.20615

Finak, G., McDavid, A., Yajima, M., Deng, J., Gersuk, V., Shalek, A. K., .. Gottardo, R. (2015). MAST: a flexible statistical framework for assessing transcriptional changes and characterizing heterogeneity in single-cell RNA sequencing data. Genome Biol, 16, 278. doi:10.1186/s13059-015-0844-5

Franke, K., Berens, P., Schubert, T., Bethge, M., Euler, T., \& Baden, T. (2017). Inhibition decorrelates visual feature representations in the inner retina. Nature, 542(7642), 439444. doi:10.1038/nature21394

Fruchterman, T. M., \& Reingold, E. M. (1991). Graph drawing by force-directed placement. Software: Practice and experience, 21(11), 1129-1164.

Fyodorov, Y. V., \& Mirlin, A. D. (1992). Analytical derivation of the scaling law for the inverse participation ratio in quasi-one-dimensional disordered systems. Physical review letters, 69(7), 1093.

Godement, P., Salaun, J., \& Imbert, M. (1984). Prenatal and postnatal development of retinogeniculate and retinocollicular projections in the mouse. J Comp Neurol, 230(4), 552-575. doi:10.1002/cne.902300406

Goetz, J., Jessen, Z. F., Jacobi, A., Mani, A., Cooler, S., Greer, D., . . Sanes, J. (2021). Unified classification of mouse retinal ganglion cells using function, morphology, and gene expression. bioRxiv.

Gomes, F. L., Zhang, G., Carbonell, F., Correa, J. A., Harris, W. A., Simons, B. D., \& Cayouette, M. (2011). Reconstruction of rat retinal progenitor cell lineages in vitro reveals a surprising degree of stochasticity in cell fate decisions. Development, 138(2), 227-235. doi:10.1242/dev.059683

Herrera, E., Brown, L., Aruga, J., Rachel, R. A., Dolen, G., Mikoshiba, K., . . Mason, C. A. (2003). Zic2 patterns binocular vision by specifying the uncrossed retinal projection. Cell, 114(5), 545-557. doi:10.1016/s0092-8674(03)00684-6

Holguera, I., \& Desplan, C. (2018). Neuronal specification in space and time. Science, 362(6411), 176-180. doi:10.1126/science.aas9435

Holt, C. E., Bertsch, T. W., Ellis, H. M., \& Harris, W. A. (1988). Cellular determination in the Xenopus retina is independent of lineage and birth date. Neuron, 1(1), 15-26. doi:10.1016/0896-6273(88)90205-x 
1993

1994

1995

1996

1997

1998

1999

2000

2001

2002

2003

2004

2005

2006

2007

2008

2009

2010

2011

2012

2013

2014

2015

2016

2017

2018

2019

2020

2021

2022

2023

2024

2025

2026

Hong, Y. K., Kim, I. J., \& Sanes, J. R. (2011). Stereotyped axonal arbors of retinal ganglion cell subsets in the mouse superior colliculus. J Comp Neurol, 519(9), 1691-1711. doi:10.1002/cne.22595

Hooks, B. M., \& Chen, C. (2020). Circuitry Underlying Experience-Dependent Plasticity in the Mouse Visual System. Neuron, 107(5), 986-987. doi:10.1016/j.neuron.2020.08.004

Jeon, C. J., Strettoi, E., \& Masland, R. H. (1998). The major cell populations of the mouse retina. J Neurosci, 18(21), 8936-8946. Retrieved from https://www.ncbi.nlm.nih.gov/pubmed/9786999

Johnson, K. P., Fitzpatrick, M. J., Zhao, L., Wang, B., McCracken, S., Williams, P. R., \& Kerschensteiner, D. (2021). Cell-type-specific binocular vision guides predation in mice. Neuron, 109(9), 1527-1539 e1524. doi:10.1016/j.neuron.2021.03.010

Johnston, R. J., Jr., \& Desplan, C. (2010). Stochastic mechanisms of cell fate specification that yield random or robust outcomes. Annu Rev Cell Dev Biol, 26, 689-719. doi:10.1146/annurev-cellbio-100109-104113

Kantorovich, L. V. (1942). On the translocation of masses. Paper presented at the Dokl. Akad. Nauk. USSR (NS).

Kay, J. N., Chu, M. W., \& Sanes, J. R. (2012). MEGF10 and MEGF11 mediate homotypic interactions required for mosaic spacing of retinal neurons. Nature, 483(7390), 465-469. doi:10.1038/nature10877

Kay, J. N., De la Huerta, I., Kim, I. J., Zhang, Y., Yamagata, M., Chu, M. W., . . Sanes, J. R. (2011). Retinal ganglion cells with distinct directional preferences differ in molecular identity, structure, and central projections. J Neurosci, 31(21), 7753-7762. doi:10.1523/JNEUROSCI.0907-11.2011

Keeley, P. W., Eglen, S. J., \& Reese, B. E. (2020). From random to regular: Variation in the patterning of retinal mosaics. J Comp Neurol, 528(13), 2135-2160. doi:10.1002/cne.24880

Kim, I. J., Zhang, Y., Meister, M., \& Sanes, J. R. (2010). Laminar restriction of retinal ganglion cell dendrites and axons: subtype-specific developmental patterns revealed with transgenic markers. J Neurosci, 30(4), 1452-1462. doi:10.1523/JNEUROSCI.477909.2010

Kiyama, T., Long, Y., Chen, C. K., Whitaker, C. M., Shay, A., Wu, H., . . Mao, C. A. (2019). Essential Roles of Tbr1 in the Formation and Maintenance of the Orientation-Selective JRGCs and a Group of OFF-Sustained RGCs in Mouse. Cell Rep, 27(3), 900-915 e905. doi:10.1016/j.celrep.2019.03.077 
2027

2028

2029

2030

2031

2032

2033

2034

2035

2036

2037

2038

2039

2040

2041

2042

2043

2044

2045

2046

2047

2048

2049

2050

2051

2052

2053

2054

2055

2056

2057

2058

Lefebvre, J. L., Sanes, J. R., \& Kay, J. N. (2015). Development of dendritic form and function. Annu Rev Cell Dev Biol, 31, 741-777. doi:10.1146/annurev-cellbio-100913-013020

Liu, J., Reggiani, J. D. S., Laboulaye, M. A., Pandey, S., Chen, B., Rubenstein, J. L. R., . . Sanes, J. R. (2018). Tbr1 instructs laminar patterning of retinal ganglion cell dendrites. Nat Neurosci, 21(5), 659-670. doi:10.1038/s41593-018-0127-z

Livesey, F. J., \& Cepko, C. L. (2001). Vertebrate neural cell-fate determination: lessons from the retina. Nat Rev Neurosci, 2(2), 109-118. doi:10.1038/35053522

Lo Giudice, Q., Leleu, M., La Manno, G., \& Fabre, P. J. (2019). Single-cell transcriptional logic of cell-fate specification and axon guidance in early-born retinal neurons. Development, 146(17). doi:10.1242/dev.178103

Lodato, S., \& Arlotta, P. (2015). Generating neuronal diversity in the mammalian cerebral cortex. Annu Rev Cell Dev Biol, 31, 699-720. doi:10.1146/annurev-cellbio-100814125353

Lyu, J., \& Mu, X. (2021). Genetic control of retinal ganglion cell genesis. Cell Mol Life Sci, 78(9), 4417-4433. doi:10.1007/s00018-021-03814-w

Macosko, E. Z., Basu, A., Satija, R., Nemesh, J., Shekhar, K., Goldman, M., .. McCarroll, S. A. (2015). Highly Parallel Genome-wide Expression Profiling of Individual Cells Using Nanoliter Droplets. Cell, 161(5), 1202-1214. doi:10.1016/j.cell.2015.05.002

Mao, C.-A., Chen, C.-K., Kiyama, T., Weber, N., Whitaker, C. M., Pan, P., . . Massey, S. C. (2020). Tbr2-expressing retinal ganglion cells are ipRGCs. bioRxiv.

Mao, C. A., Li, H., Zhang, Z., Kiyama, T., Panda, S., Hattar, S., . . Wang, S. W. (2014). T-box transcription regulator Tbr2 is essential for the formation and maintenance of Opn4/melanopsin-expressing intrinsically photosensitive retinal ganglion cells. J Neurosci, 34(39), 13083-13095. doi:10.1523/JNEUROSCI.1027-14.2014

Marcucci, F., Soares, C. A., \& Mason, C. (2019). Distinct timing of neurogenesis of ipsilateral and contralateral retinal ganglion cells. J Comp Neurol, 527(1), 212-224. doi:10.1002/cne.24467

Marquardt, T., \& Gruss, P. (2002). Generating neuronal diversity in the retina: one for nearly all. Trends Neurosci, 25(1), 32-38. doi:10.1016/s0166-2236(00)02028-2

Martersteck, E. M., Hirokawa, K. E., Evarts, M., Bernard, A., Duan, X., Li, Y., . . Harris, J. A. (2017). Diverse Central Projection Patterns of Retinal Ganglion Cells. Cell Rep, 18(8), 2058-2072. doi:10.1016/j.celrep.2017.01.075 
2059

2060

2061

2062

2063

2064

2065

2066

2067

2068

2069

2070

2071

2072

2073

2074

2075

2076

2077

2078

2079

2080

2081

2082

2083

2084

2085

2086

2087

2088

2089

2090

Masland, R. H. (2012). The neuronal organization of the retina. Neuron, 76(2), 266-280. doi:10.1016/j.neuron.2012.10.002

Mason, C., \& Slavi, N. (2020). Retinal Ganglion Cell Axon Wiring Establishing the Binocular Circuit. Annu Rev Vis Sci, 6, 215-236. doi:10.1146/annurev-vision-091517-034306

McConnell, S. K. (1991). The generation of neuronal diversity in the central nervous system. Annu Rev Neurosci, 14, 269-300. doi:10.1146/annurev.ne.14.030191.001413

McKenna, A., Findlay, G. M., Gagnon, J. A., Horwitz, M. S., Schier, A. F., \& Shendure, J. (2016). Whole-organism lineage tracing by combinatorial and cumulative genome editing. Science, 353(6298), aaf7907. doi:10.1126/science.aaf7907

McNeill, D. S., Sheely, C. J., Ecker, J. L., Badea, T. C., Morhardt, D., Guido, W., \& Hattar, S. (2011). Development of melanopsin-based irradiance detecting circuitry. Neural Dev, 6 , 8. doi:10.1186/1749-8104-6-8

Monge, G. (1781). Mémoire sur la théorie des déblais et des remblais. Histoire de l'Académie Royale des Sciences de Paris.

Osterhout, J. A., Josten, N., Yamada, J., Pan, F., Wu, S. W., Nguyen, P. L., . . Huberman, A. D. (2011). Cadherin-6 mediates axon-target matching in a non-image-forming visual circuit. Neuron, 71(4), 632-639. doi:10.1016/j.neuron.2011.07.006

Pak, W., Hindges, R., Lim, Y. S., Pfaff, S. L., \& O'Leary, D. D. (2004). Magnitude of binocular vision controlled by islet-2 repression of a genetic program that specifies laterality of retinal axon pathfinding. Cell, 119(4), 567-578. doi:10.1016/j.cell.2004.10.026

Peng, Y. R., Tran, N. M., Krishnaswamy, A., Kostadinov, D., Martersteck, E. M., \& Sanes, J. R. (2017). Satb1 Regulates Contactin 5 to Pattern Dendrites of a Mammalian Retinal Ganglion Cell. Neuron, 95(4), 869-883 e866. doi:10.1016/j.neuron.2017.07.019

Price, J., Turner, D., \& Cepko, C. (1987). Lineage analysis in the vertebrate nervous system by retrovirus-mediated gene transfer. Proc Natl Acad Sci U S A, 84(1), 156-160. doi:10.1073/pnas.84.1.156

Rheaume, B. A., Jereen, A., Bolisetty, M., Sajid, M. S., Yang, Y., Renna, K., . . Trakhtenberg, E. F. (2018). Single cell transcriptome profiling of retinal ganglion cells identifies cellular subtypes. Nat Commun, 9(1), 2759. doi:10.1038/s41467-018-05134-3

Rockhill, R. L., Euler, T., \& Masland, R. H. (2000). Spatial order within but not between types of retinal neurons. Proc Natl Acad Sci U S A, 97(5), 2303-2307. doi:10.1073/pnas.030413497 
2091

2092

2093

2094

2095

2096

2097

2098

2099

2100

2101

2102

2103

2104

2105

2106

2107

2108

2109

2110

2111

2112

2113

2114

2115

2116

2117

2118

2119

2120

2121

2122

2123

2124

Rousso, D. L., Qiao, M., Kagan, R. D., Yamagata, M., Palmiter, R. D., \& Sanes, J. R. (2016). Two Pairs of ON and OFF Retinal Ganglion Cells Are Defined by Intersectional Patterns of Transcription Factor Expression. Cell Rep, 15(9), 1930-1944. doi:10.1016/j.celrep.2016.04.069

Sagner, A., \& Briscoe, J. (2019). Establishing neuronal diversity in the spinal cord: a time and a place. Development, 146(22). doi:10.1242/dev.182154

Sajgo, S., Ghinia, M. G., Brooks, M., Kretschmer, F., Chuang, K., Hiriyanna, S., . . Badea, T. C. (2017). Molecular codes for cell type specification in Brn3 retinal ganglion cells. Proc Natl Acad Sci U S A, 114(20), E3974-E3983. doi:10.1073/pnas.1618551114

Sanes, J. R., \& Masland, R. H. (2015). The types of retinal ganglion cells: current status and implications for neuronal classification. Annu Rev Neurosci, 38, 221-246. doi:10.1146/annurev-neuro-071714-034120

Sanes, J. R., Rubenstein, J. L., \& Nicolas, J. F. (1986). Use of a recombinant retrovirus to study post-implantation cell lineage in mouse embryos. EMBO J, 5(12), 3133-3142. Retrieved from https://www.ncbi.nlm.nih.gov/pubmed/3102226

Schiebinger, G., Shu, J., Tabaka, M., Cleary, B., Subramanian, V., Solomon, A., . . Lander, E. S. (2019). Optimal-Transport Analysis of Single-Cell Gene Expression Identifies Developmental Trajectories in Reprogramming. Cell, 176(6), 1517. doi:10.1016/j.cell.2019.02.026

Sharma, N., Flaherty, K., Lezgiyeva, K., Wagner, D. E., Klein, A. M., \& Ginty, D. D. (2020). The emergence of transcriptional identity in somatosensory neurons. Nature, 577(7790), 392-398. doi:10.1038/s41586-019-1900-1

Shekhar, K., Lapan, S. W., Whitney, I. E., Tran, N. M., Macosko, E. Z., Kowalczyk, M., . . Sanes, J. R. (2016). Comprehensive Classification of Retinal Bipolar Neurons by SingleCell Transcriptomics. Cell, 166(5), 1308-1323 e1330. doi:10.1016/j.cell.2016.07.054

Shekhar, K., \& Sanes, J. R. (2021). Generating and Using Transcriptomically Based Retinal Cell Atlases. Annu Rev Vis Sci. doi:10.1146/annurev-vision-032621-075200

Soares, C. A., \& Mason, C. A. (2015). Transient ipsilateral retinal ganglion cell projections to the brain: Extent, targeting, and disappearance. Dev Neurobiol, 75(12), 1385-1401. doi:10.1002/dneu.22291

Stehman, S. V. (1997). Selecting and interpreting measures of thematic classification accuracy. Remote sensing of Environment, 62(1), 77-89.

Tran, N. M., Shekhar, K., Whitney, I. E., Jacobi, A., Benhar, I., Hong, G., . . Sanes, J. R. (2019). Single-Cell Profiles of Retinal Ganglion Cells Differing in Resilience to Injury 
Reveal Neuroprotective Genes. Neuron, 104(6), 1039-1055 e1012. doi:10.1016/j.neuron.2019.11.006

Trimarchi, J. M., Stadler, M. B., \& Cepko, C. L. (2008). Individual retinal progenitor cells display extensive heterogeneity of gene expression. PLoS One, 3(2), e1588. doi:10.1371/journal.pone.0001588

Turner, D. L., \& Cepko, C. L. (1987). A common progenitor for neurons and glia persists in rat retina late in development. Nature, 328(6126), 131-136. doi:10.1038/328131a0

Turner, D. L., Snyder, E. Y., \& Cepko, C. L. (1990). Lineage-independent determination of cell type in the embryonic mouse retina. Neuron, 4(6), 833-845. doi:10.1016/08966273(90)90136-4

Villani, C. (2009). Optimal transport: old and new (Vol. 338): Springer.

Voinescu, P. E., Kay, J. N., \& Sanes, J. R. (2009). Birthdays of retinal amacrine cell subtypes are systematically related to their molecular identity and soma position. J Comp Neurol, 517(5), 737-750. doi:10.1002/cne.22200

Wamsley, B., \& Fishell, G. (2017). Genetic and activity-dependent mechanisms underlying interneuron diversity. Nat Rev Neurosci, 18(5), 299-309. doi:10.1038/nrn.2017.30

Wang, Q., Marcucci, F., Cerullo, I., \& Mason, C. (2016). Ipsilateral and Contralateral Retinal Ganglion Cells Express Distinct Genes during Decussation at the Optic Chiasm. eNeuro, 3(6). doi:10.1523/ENEURO.0169-16.2016

Welch, J. D., Kozareva, V., Ferreira, A., Vanderburg, C., Martin, C., \& Macosko, E. Z. (2019). Single-Cell Multi-omic Integration Compares and Contrasts Features of Brain Cell Identity. Cell, 177(7), 1873-1887 e1817. doi:10.1016/j.cell.2019.05.006

Wetts, R., \& Fraser, S. E. (1988). Multipotent precursors can give rise to all major cell types of the frog retina. Science, 239(4844), 1142-1145. doi:10.1126/science.2449732

Yamagata, M., Yan, W., \& Sanes, J. R. (2021). A cell atlas of the chick retina based on singlecell transcriptomics. Elife, 10. doi:10.7554/eLife.63907

Yan, W., Laboulaye, M. A., Tran, N. M., Whitney, I. E., Benhar, I., \& Sanes, J. R. (2020). Mouse Retinal Cell Atlas: Molecular Identification of over Sixty Amacrine Cell Types. J Neurosci, 40(27), 5177-5195. doi:10.1523/JNEUROSCI.0471-20.2020

Yuste, R., Hawrylycz, M., Aalling, N., Aguilar-Valles, A., Arendt, D., Armananzas, R., . . Lein, E. (2020). A community-based transcriptomics classification and nomenclature of neocortical cell types. Nat Neurosci, 23(12), 1456-1468. doi:10.1038/s41593-020-0685-8 
2157 Zeng, H., \& Sanes, J. R. (2017). Neuronal cell-type classification: challenges, opportunities and 2158 the path forward. Nat Rev Neurosci, 18(9), 530-546. doi:10.1038/nrn.2017.85

2159 Zheng, G. X., Terry, J. M., Belgrader, P., Ryvkin, P., Bent, Z. W., Wilson, R., . . Bielas, J. H. 2160 (2017). Massively parallel digital transcriptional profiling of single cells. Nat Commun, 8, 2161 14049. doi:10.1038/ncomms 14049

2162

2163

2164

2165

2166 
Supplementary Fig. 1

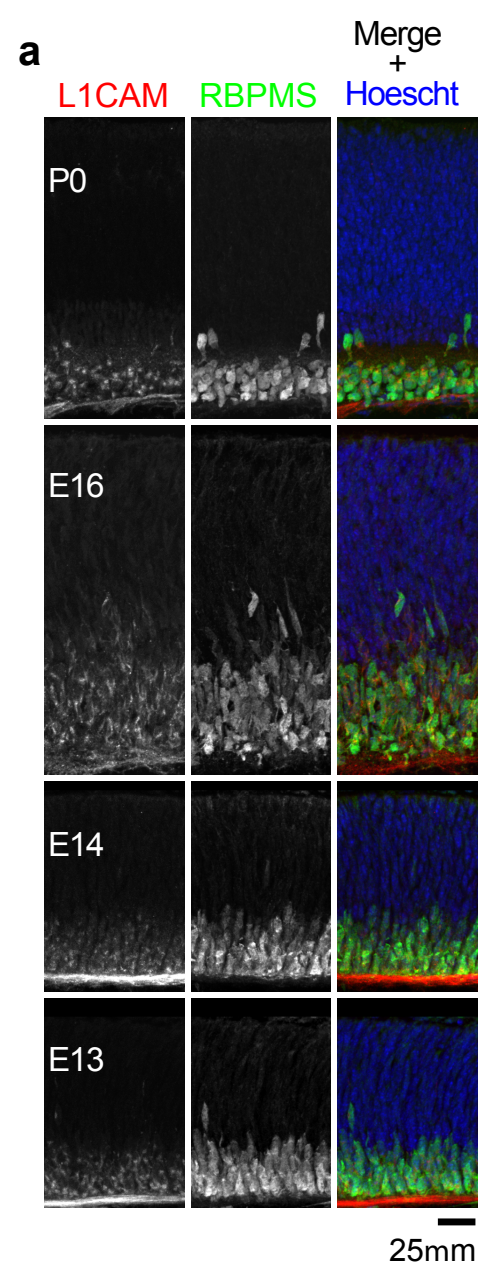

b

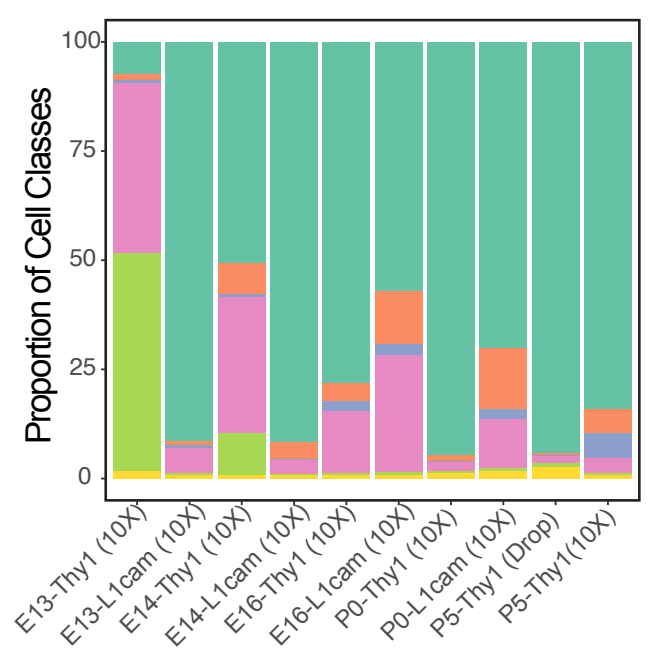

C

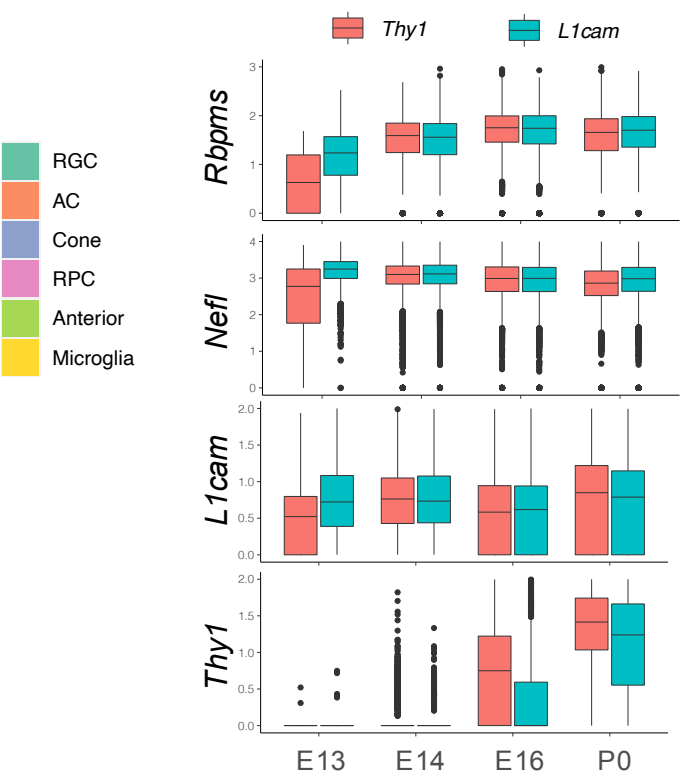

d

e
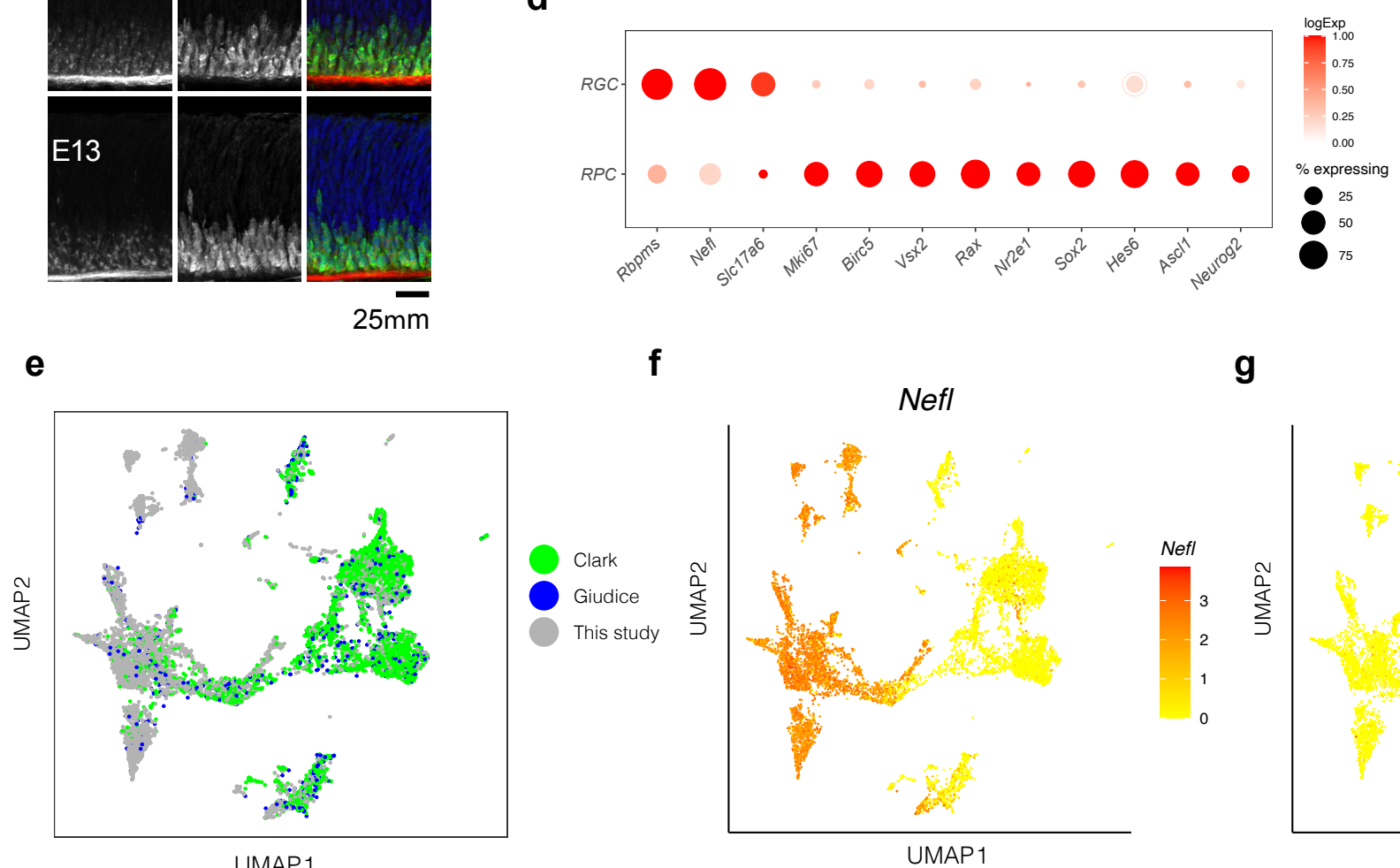

UMAP1

h

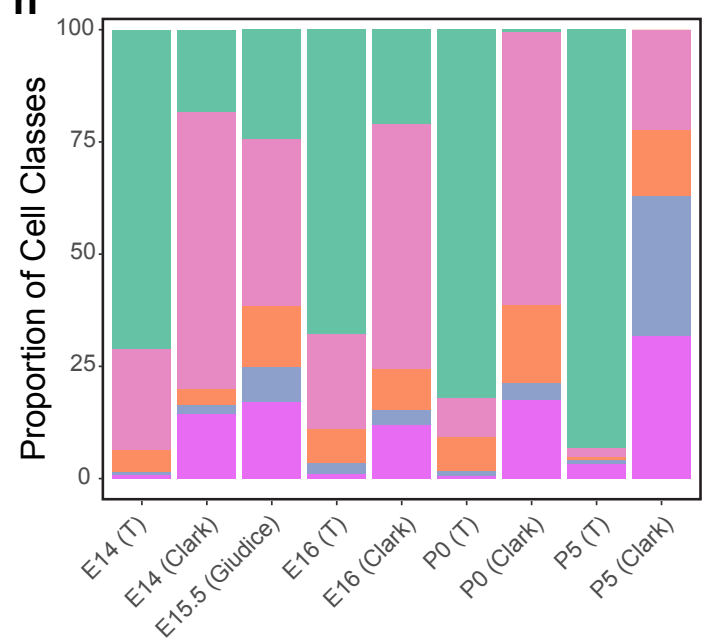

Fgf15

g
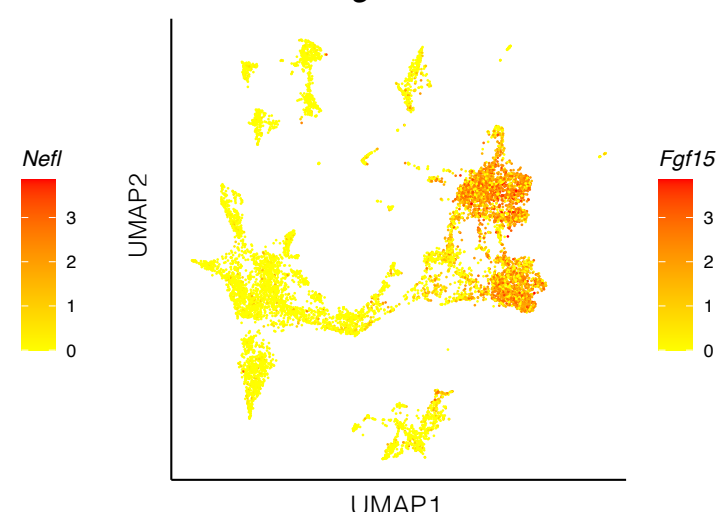

UMAP1
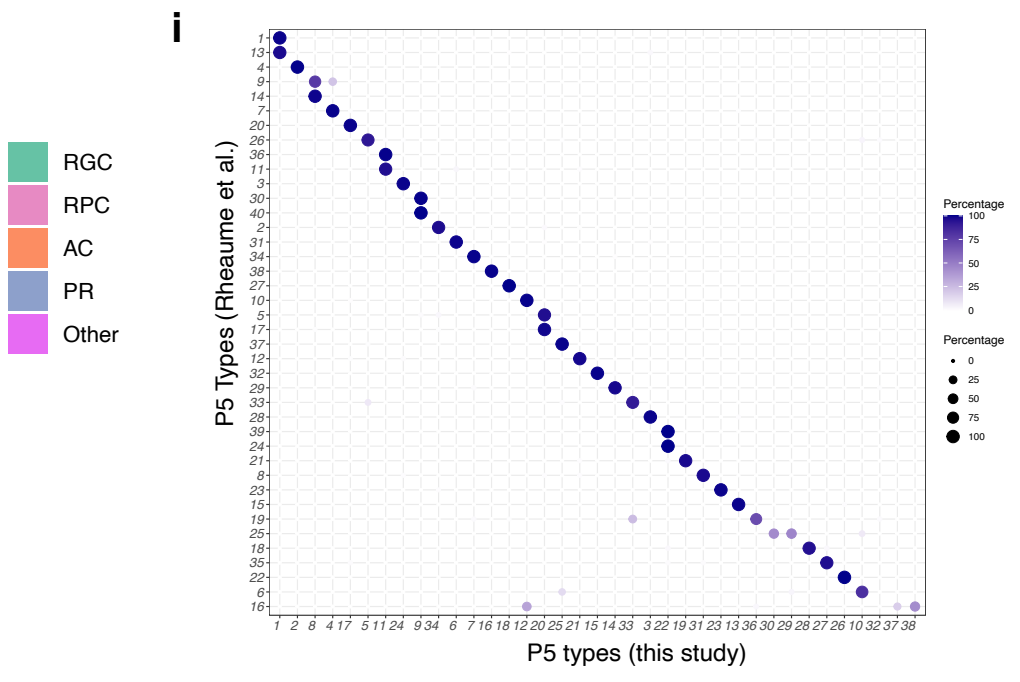
Supplementary Fig. 2

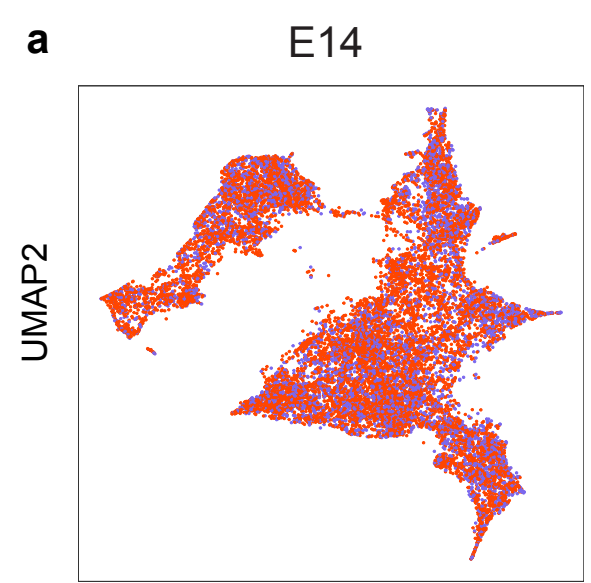

UMAP1 b

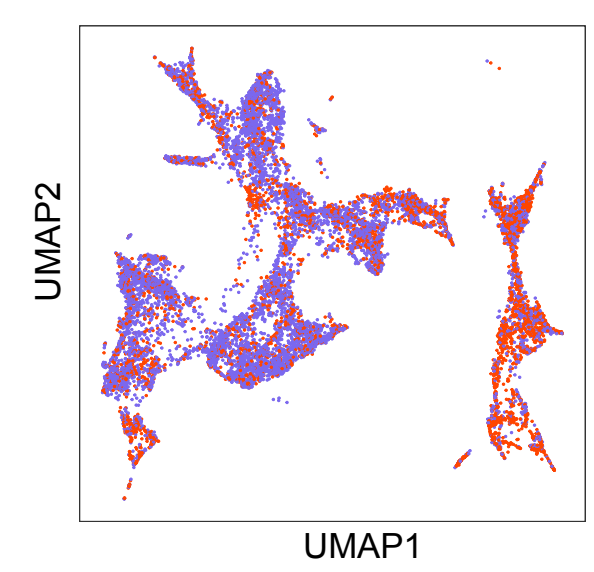

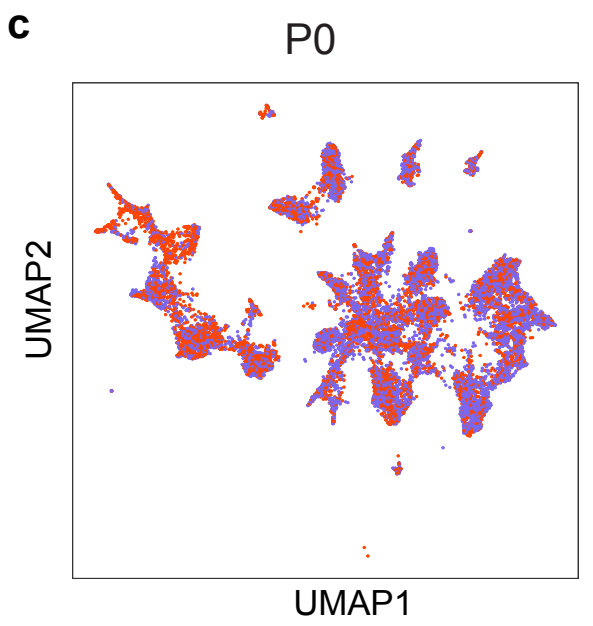

d

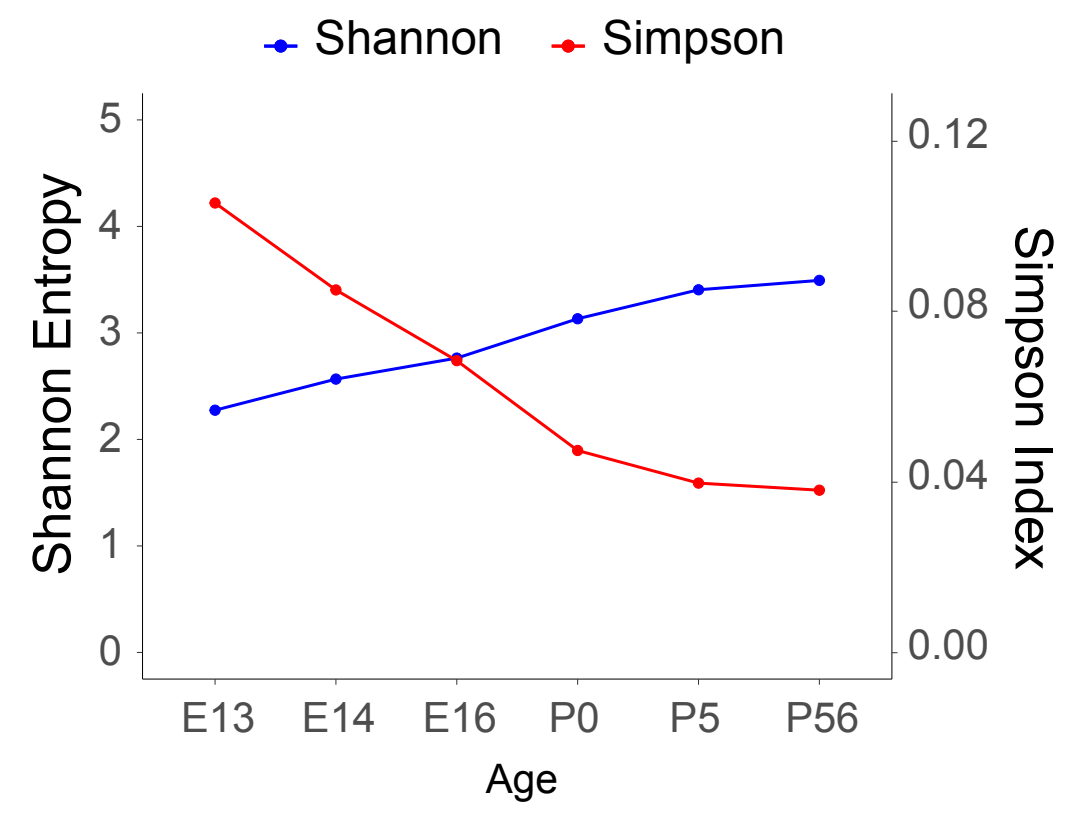




\section{Supplementary Fig. 3}

a

b

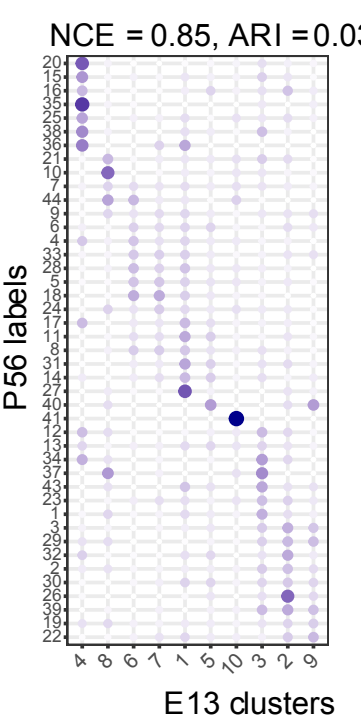

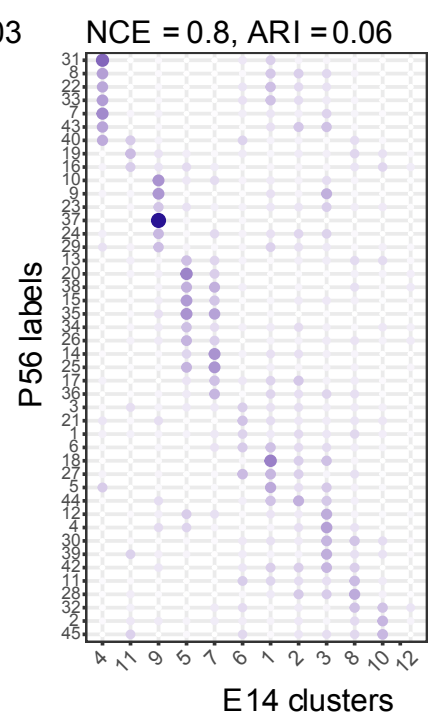

C

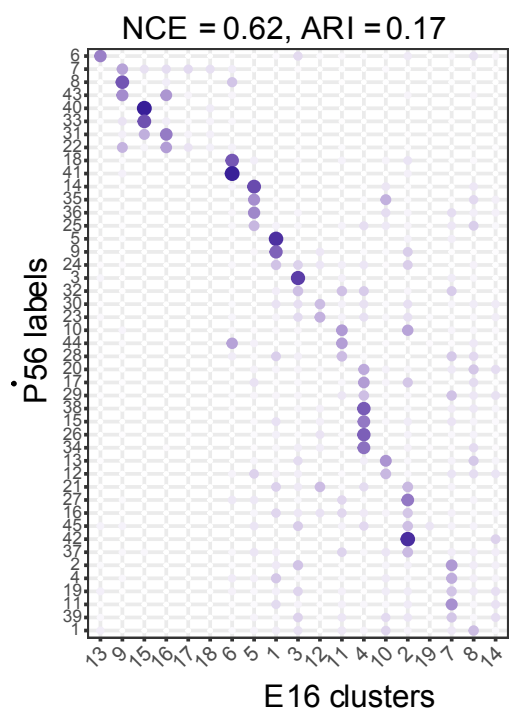

d

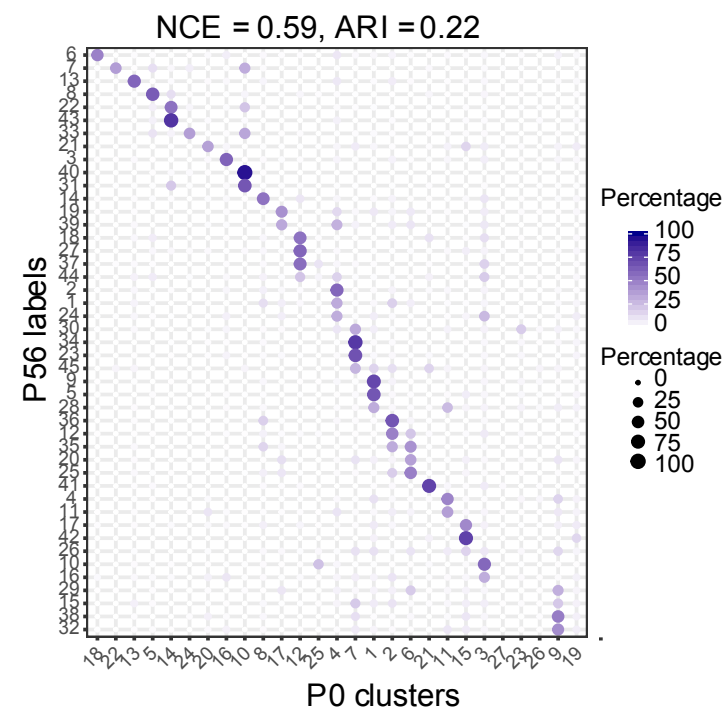

e

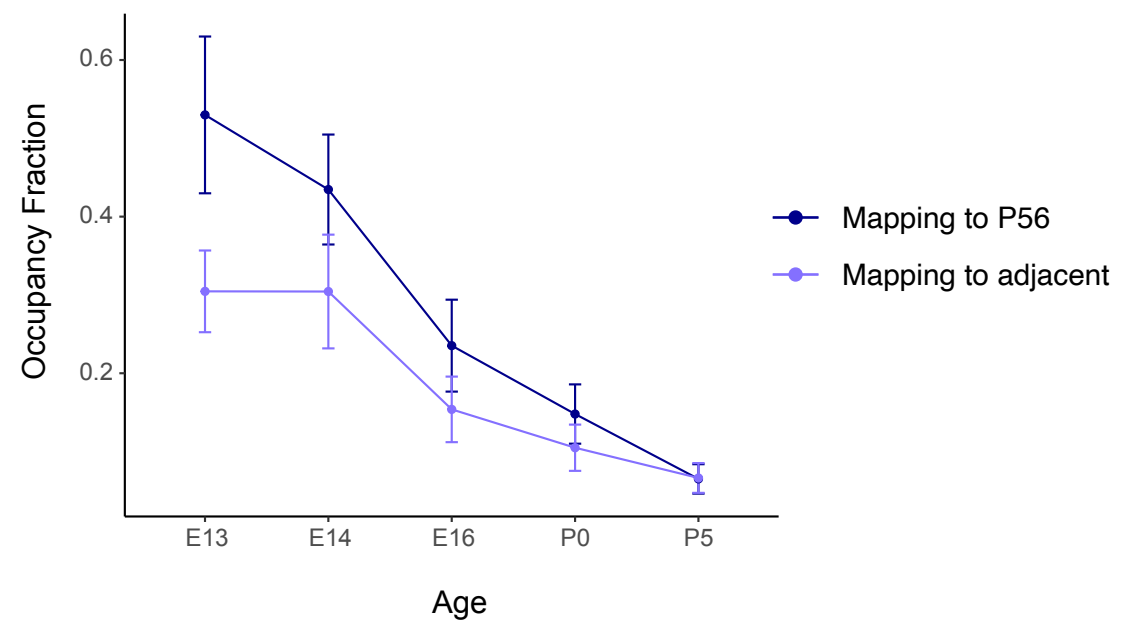


a

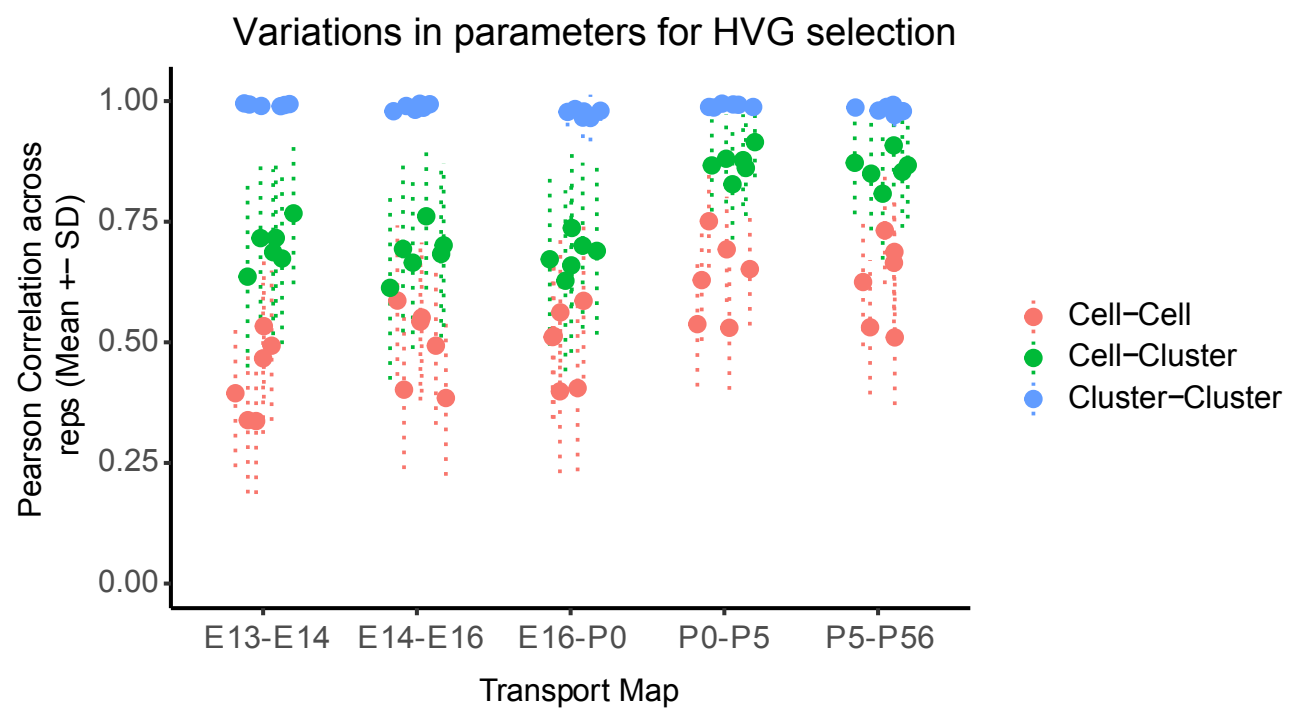

b Variations in $\varepsilon$

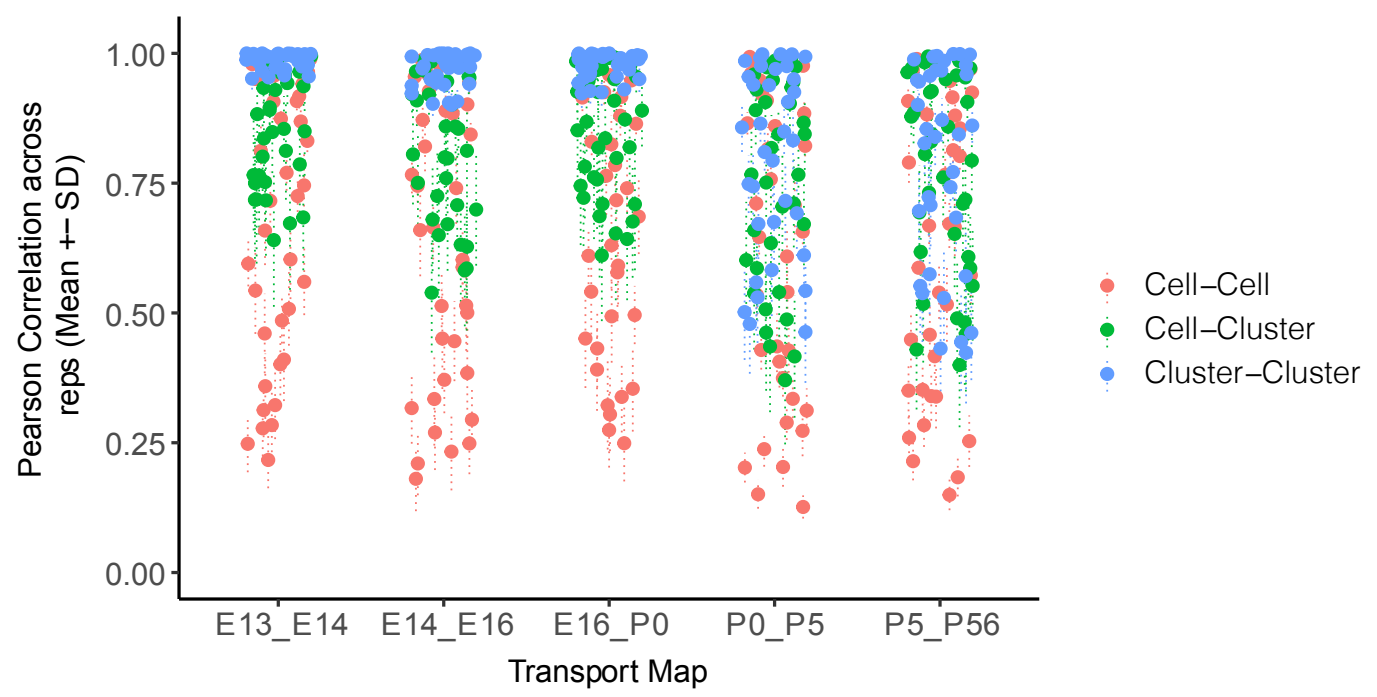

C Correlation of transport couplings at the level of clusters

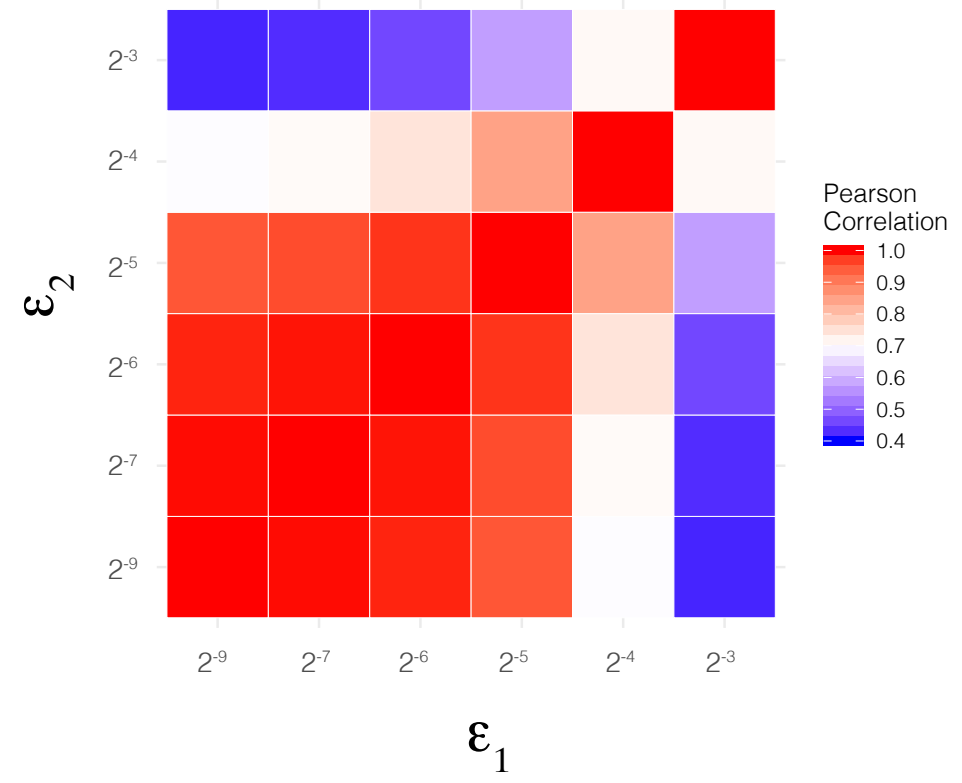


Supplementary Fig. 5

a

Corr with Figure 3D $=0.96$

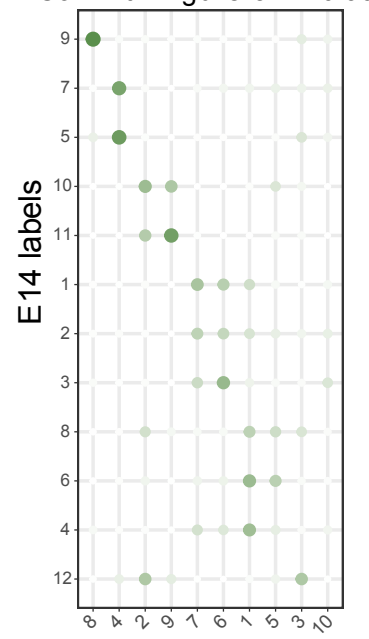

E13 clusters b

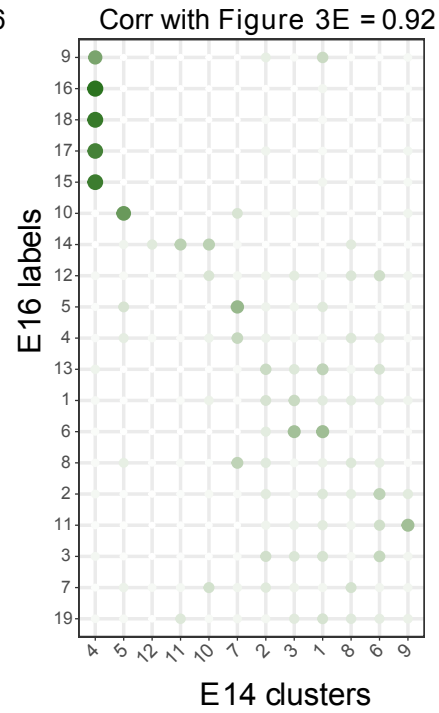

C

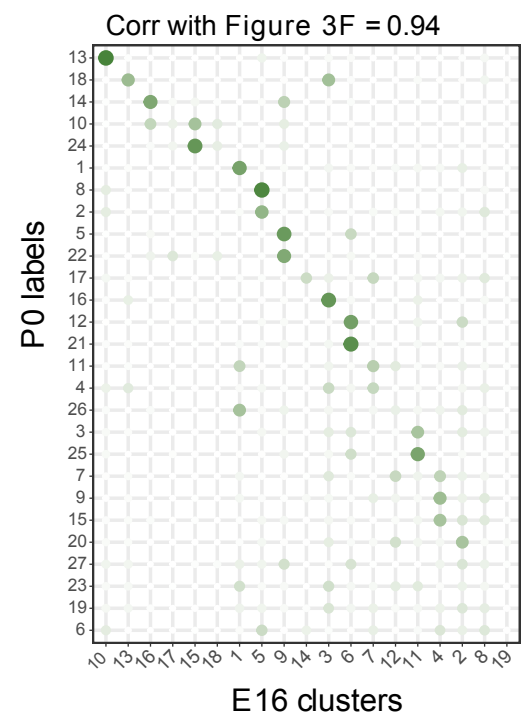

d

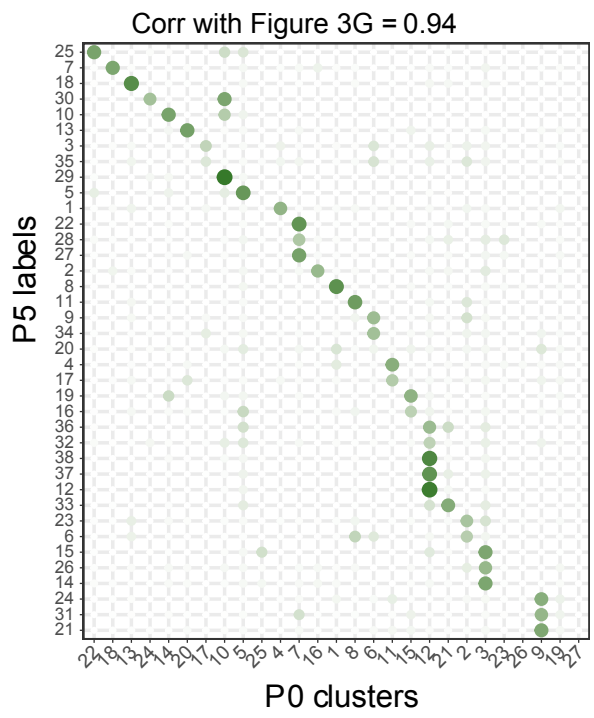

e

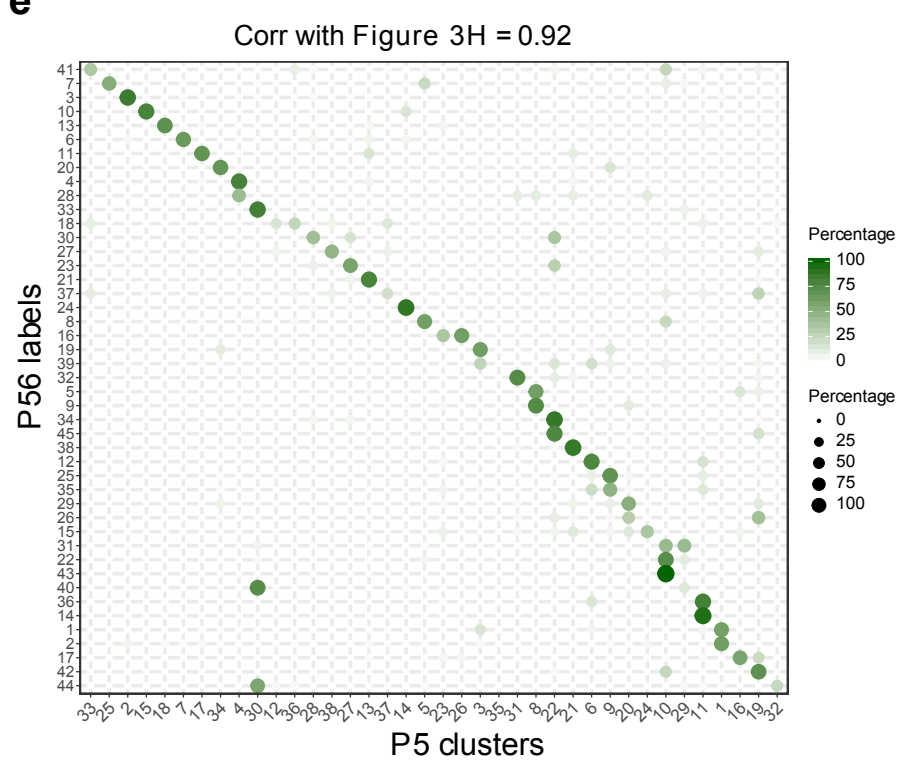




\section{Supplementary Figure 6}

a
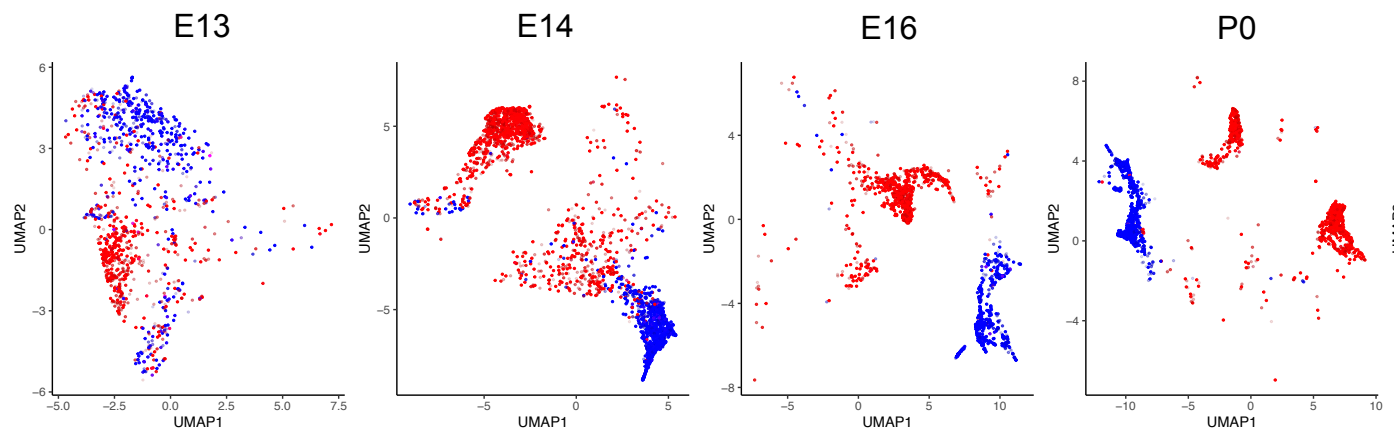

E16

b
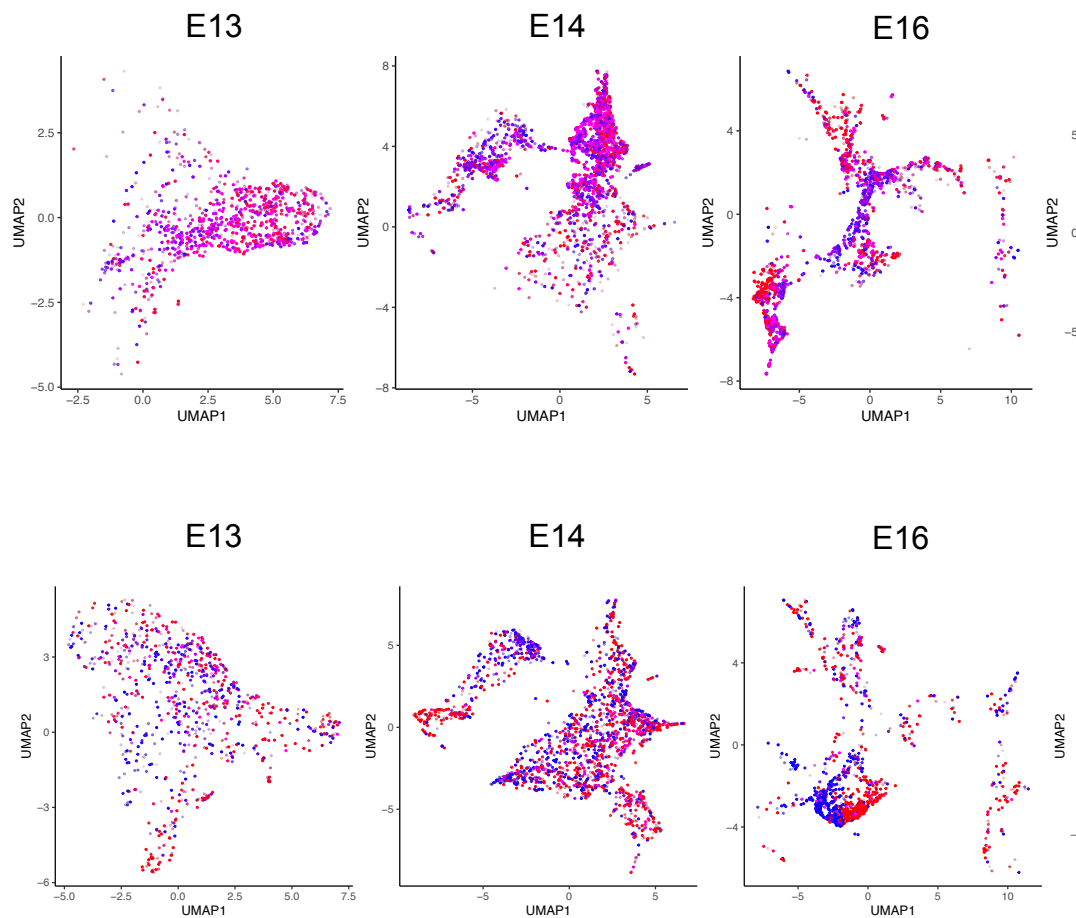

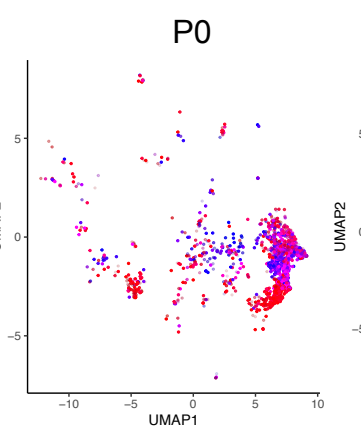

PO
E13

E14
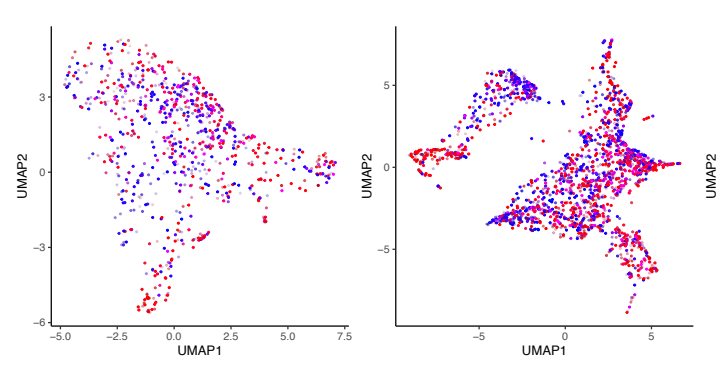

C
E16

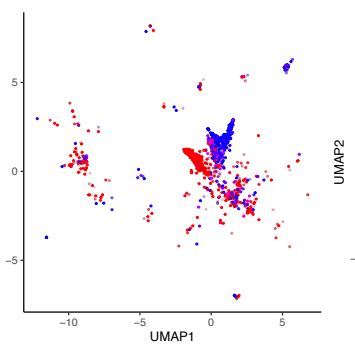

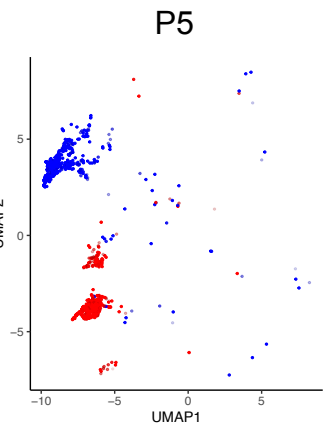

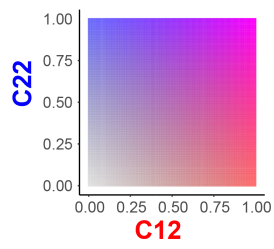

P5
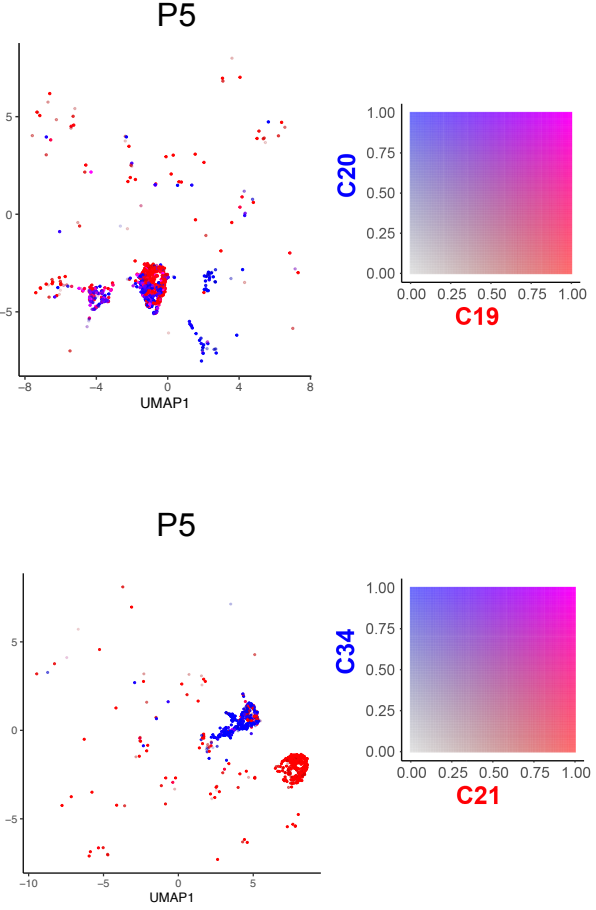

d

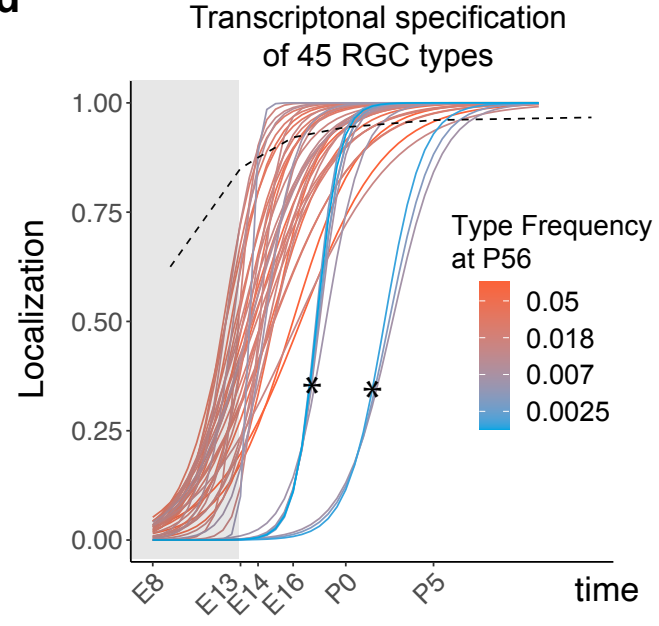


a

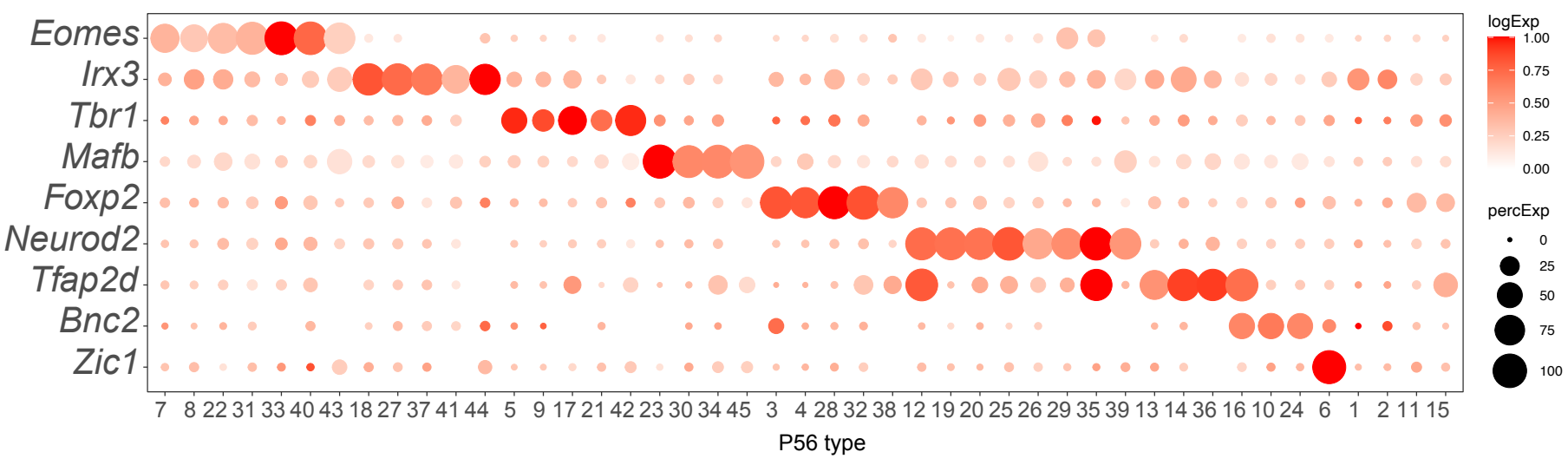

b

Tbr1 types

Avg. $\tau_{\mathrm{sp}}=\mathrm{E} 17.8$

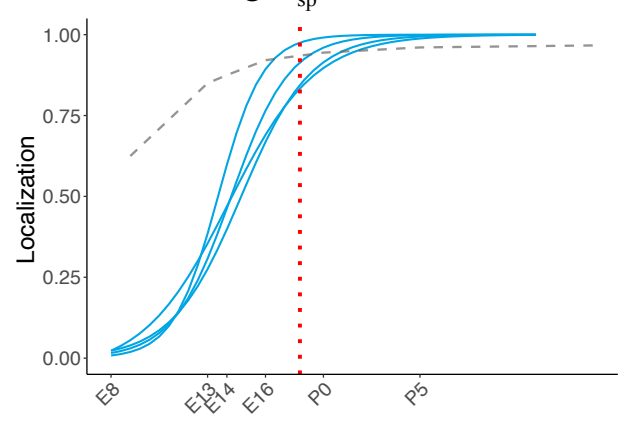

e

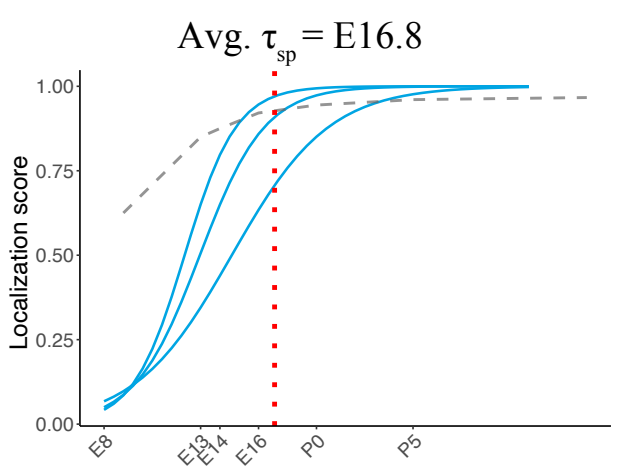

C

Tfap2d types

Avg. $\tau_{\mathrm{sp}}=\mathrm{E} 16.5$

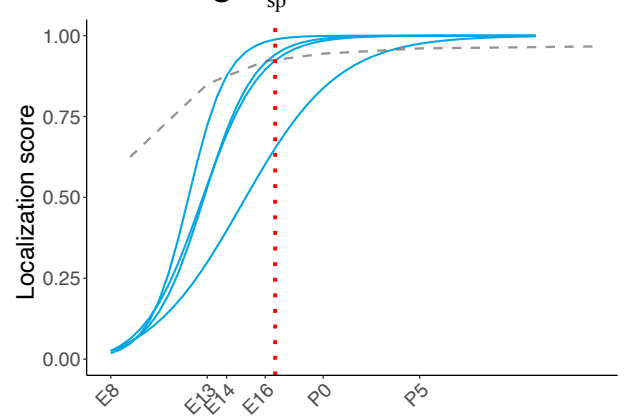

f

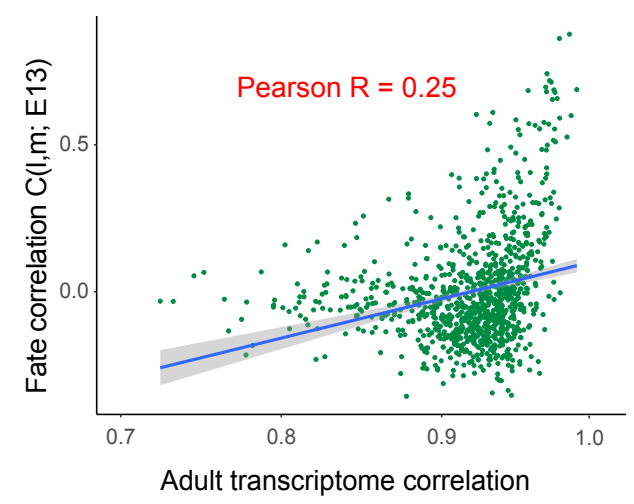

d Foxp2 types

Avg. $\tau_{\mathrm{sp}}=\mathrm{E} 17.2$

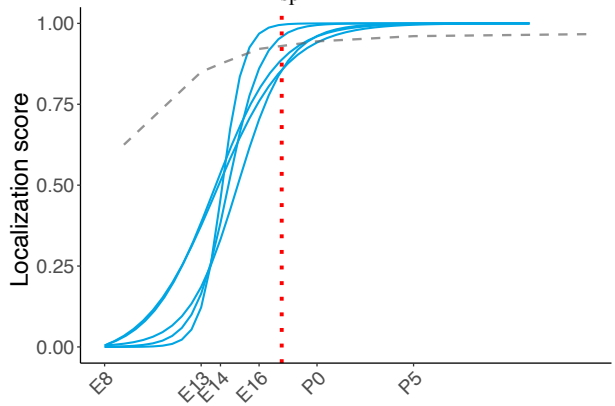

9

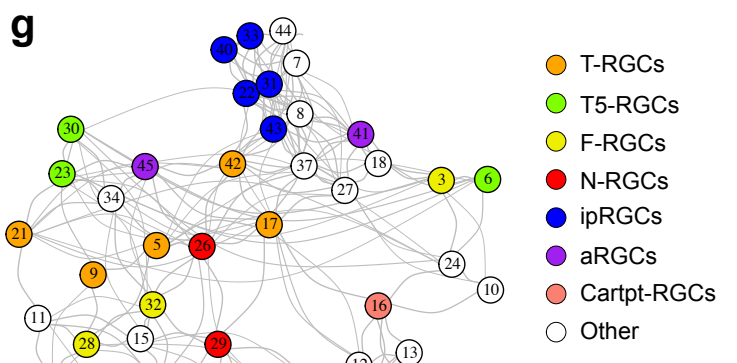




\section{Supplementary Figure 8}
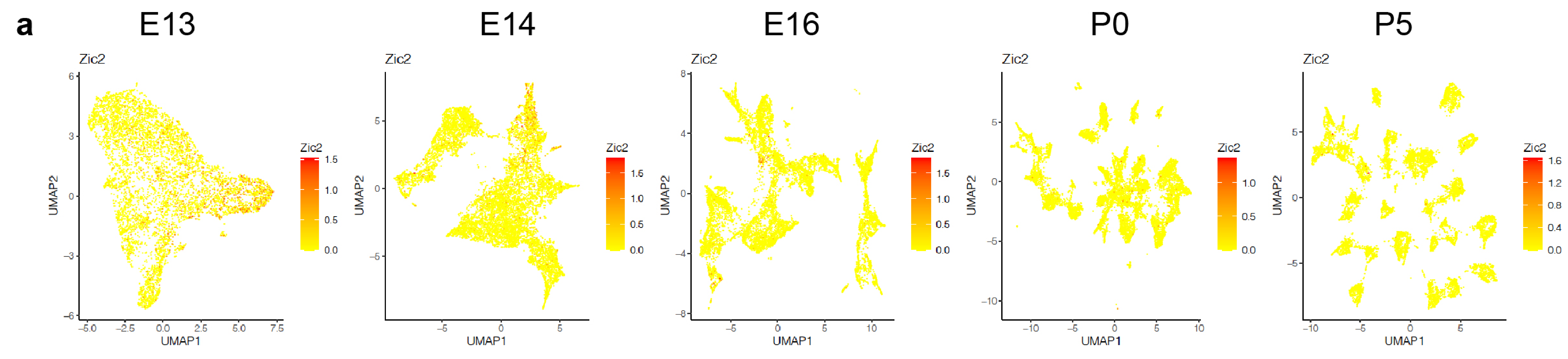

b

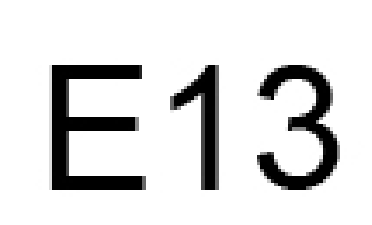

E14

E16
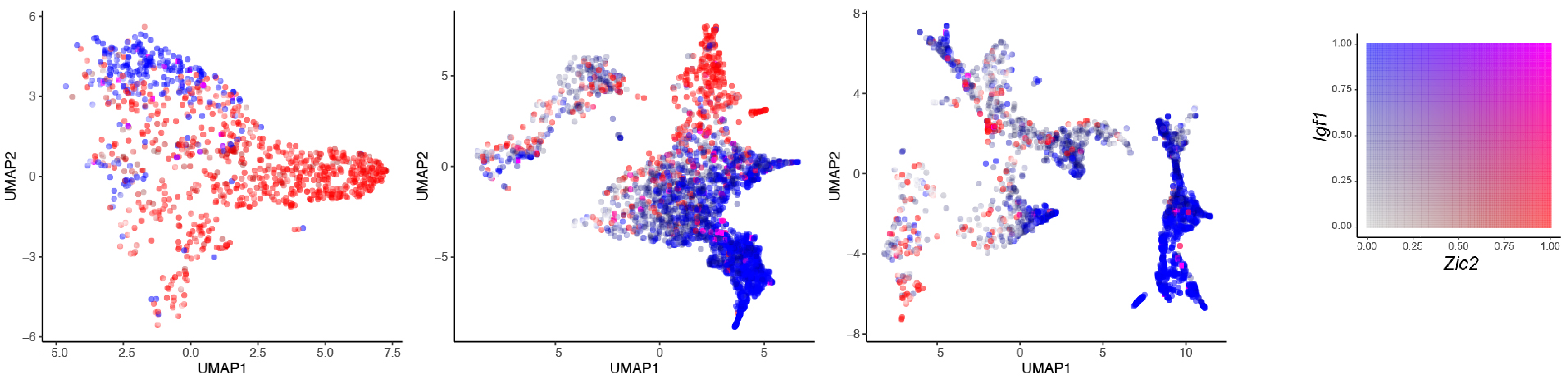

C
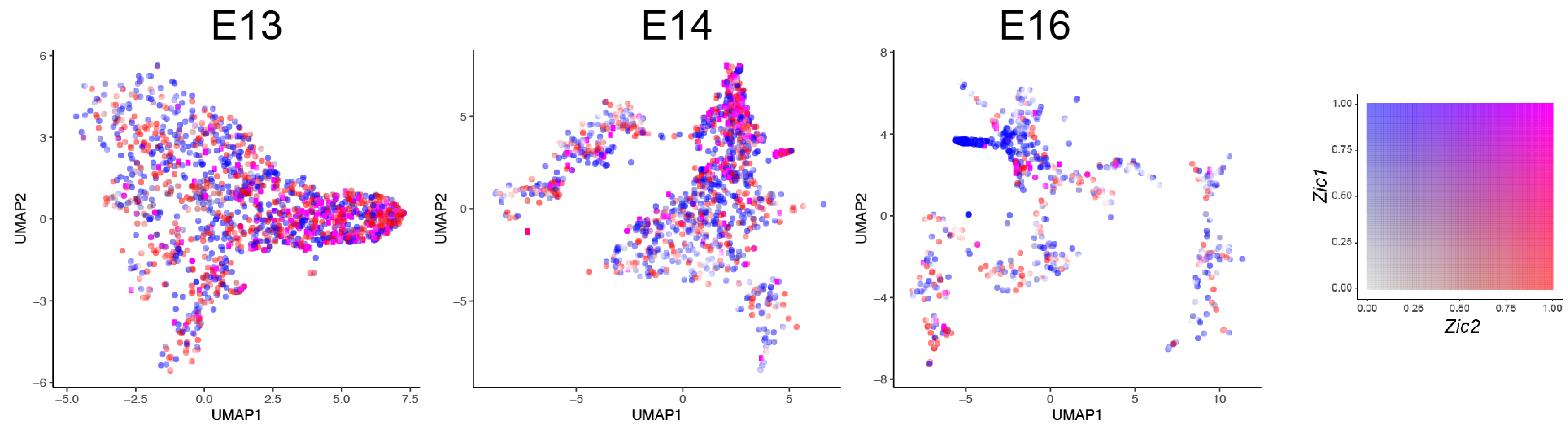

d
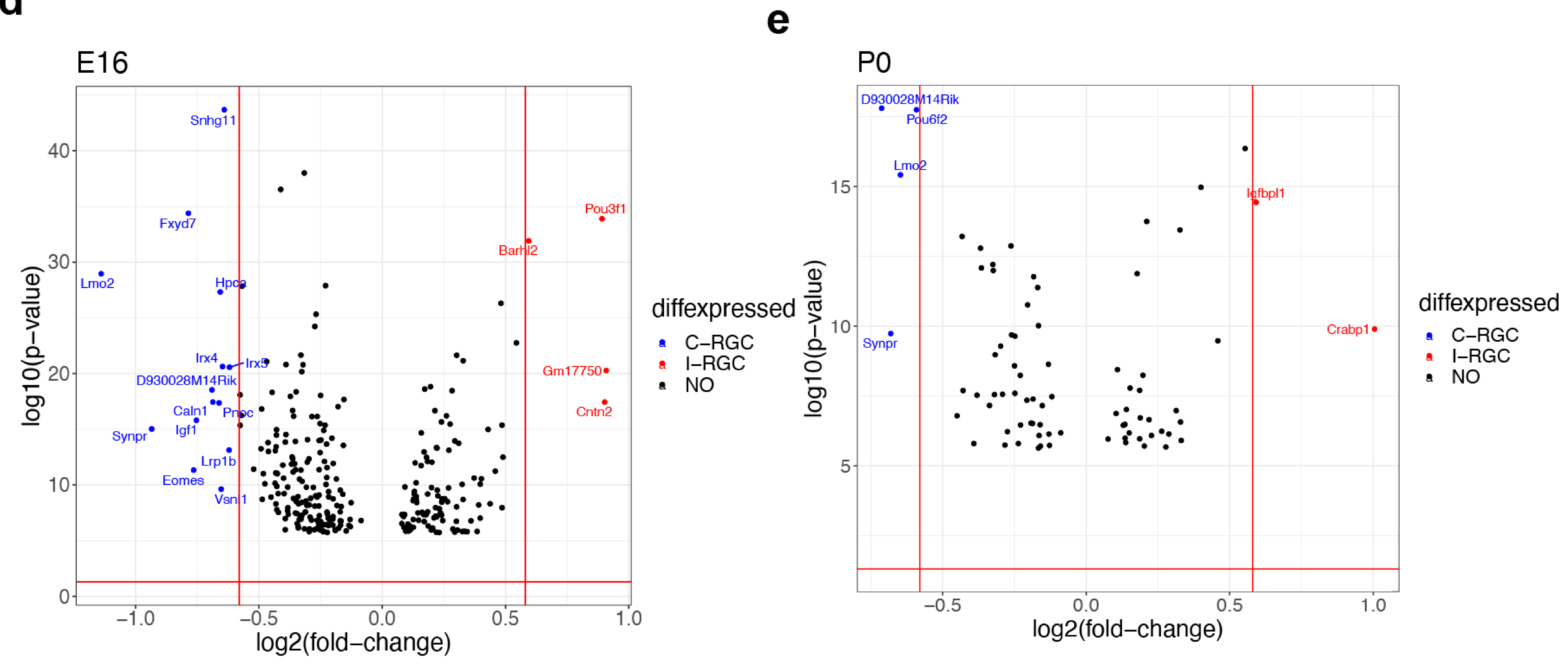